Florida International University FIU Digital Commons

$11-3-2016$

\title{
Two Dimensional Lattice Gauge Theory with and without Fermion Content
}

Dibakar Sigdel

FIU, dsigd001@fiu.edu

DOI: $10.25148 /$ etd.FIDC001748

Follow this and additional works at: https://digitalcommons.fiu.edu/etd

Part of the Physics Commons

\section{Recommended Citation}

Sigdel, Dibakar, "Two Dimensional Lattice Gauge Theory with and without Fermion Content" (2016). FIU Electronic Theses and Dissertations. 3224.

https://digitalcommons.fiu.edu/etd/3224

This work is brought to you for free and open access by the University Graduate School at FIU Digital Commons. It has been accepted for inclusion in FIU Electronic Theses and Dissertations by an authorized administrator of FIU Digital Commons. For more information, please contact dcc@fiu.edu. 


\section{FLORIDA INTERNATIONAL UNIVERSITY}

Miami, Florida

TWO DIMENSIONAL LATTICE GAUGE THEORY WITH AND WITHOUT FERMION CONTENT

A dissertation submitted in partial fulfillment of the

requirements of the degree of

DOCTOR OF PHILOSOPHY

in

PHYSICS

by

Dibakar Sigdel

2017 
To: Dean Michael R. Heithaus

College of Arts, Sciences and Education

This dissertation, written by Dibakar Sigdel, and entitled Two Dimensional Lattice Gauge Theory with and without Fermion Content, having been approved in respect to style and intellectual content, is referred to you for judgment.

We have read this dissertation and recommend that it be approved.

$\begin{array}{r}\text { H Rudolf Fiebig } \\ \hline \text { Misak M Sargsian } \\ \hline \text { Gueo Grantcharov } \\ \hline \text { Rajamani Narayanan, Major Professor }\end{array}$

Date of Defense: November 3, 2016

The dissertation of Dibakar Sigdel is approved.

Dean Michael R. Heithaus

College of Arts, Sciences and Education

Andrés G. Gil

Vice President for Research and Economic Development and Dean of the University Graduate School

Florida International University, 2017 


\section{DEDICATION}

This dissertation is dedicated to my parents. 


\section{ACKNOWLEDGMENTS}

First of all, I would like to express my sincere gratitude to my advisor Professor Dr. Rajamani Narayanan for his continuous academic guidance of my Ph.D study, for his motivation, and immense knowledge. Besides my advisor, I would like to thank the rest of my dissertation committee: Dr. Gueo Grantcharov for helping me to appreciate the depths of pure mathematics, Dr. Misak M Sargsian for explanations on different aspects of perturbative Quamtum Field Theory; Dr. H Rudolf Fiebig for showing me the importance of different techniques of Computational Physics and Algorithms.

I wish to acknowledge the past and present graduate program director, Brian Raue and Jorge L. Rodriguez, as well as many of the past and present secretaries of the FIU physics department, including Elizabeth Bergano-Smith, Omar Tolbert, Maria Martinez, Ofelia Adan-Fernandez, and Robert Brown for systemetizing all official task in my graduate student experience.

I additionally acknowledge Nikhil Karthik, Rafael Badui, Oswaldo Artiles, Shankar Dayal Adhikari, Hari Khanal and Ricardo Leante for the absolutely fascinating physics discussions that I have had with them.

I too must acknowledge my parents for their support and encouragement. This research would not have been possible without financial support. I would like to acknowledge NSF for providing the grant that supported my research assistantship.

Lastly, I acknowledge my wife Namuna Panday for her support and encouragements in each and every steps during my graduate school days. 


\title{
ABSTRACT OF THE DISSERTATION \\ TWO DIMENSIONAL LATTICE GAUGE THEORY WITH AND WITHOUT \\ FERMION CONTENT
}

by

\author{
Dibakar Sigdel
}

Florida International University, 2017

Miami, Florida

Professor Rajamani Narayanan, Major Professor

Quantum Chromo Dyamics (QCD) is a relativistic field theory of a non-abelian gauge field coupled to several flavors of fermions. Two dimensional (one space and one time) QCD serves as an interesting toy model that shares several features with the four dimensional physically relevant theory. The main aim of the research is to study two dimensional QCD using the lattice regularization.

Two dimensional QCD without any fermion content is solved analytically using lattice regularization. Explicit expressions for the expectation values of Wilson loops and the correlation of two Polyakov loops oriented in two different directions are obtained. Physics of the QCD vacuum is explained using these results.

The Hamlitonian formalism of lattice QCD with fermion content serves as an approach to study quark excitations out of the vacuum. The formalism is first devloped and techniques to numerically evaluate the spectrum of physical particles, namely, meson and baryons are described. The Hybrid Monte Carlo technique was used to numerically extract the lowest meson and baryon masses as a function of the quark masses. It is shown that neither the lowest meson mass nor the lowest baryon mass goes to zero as the quark mass is taken to zero. This numerically establishes the presence of a mass gap in two dimensional QCD. 


\section{TABLE OF CONTENTS}

\section{CHAPTER}

PAGE

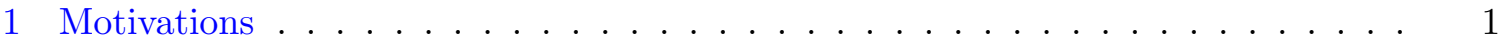

2 Basics of gauge theory on the lattice . . . . . . . . . . . . . . 4

2.1 Introduction . . . . . . . . . . . . . . . . . . 4

2.2 Wilson loops and Polyakov loops . . . . . . . . . . . . . . . . 7

2.3 Continuum limit . . . . . . . . . . . . . . . . . . . . . . . 10

3 Two dimensional lattice gauge theory without fermion content . . . . . . . . . 12

3.1 Introduction . . . . . . . . . . . . . . . . . . . 12

3.2 Gauge field configuration . . . . . . . . . . . . . . . . . 13

3.3 Partition function . . . . . . . . . . . . . . . . 16

4 Observables and results from analytical calculations . . . . . . . . . . . . . 19

4.1 Wilson loops . . . . . . . . . . . . . . . . . . . . . . 19

4.2 Polyakov loops . . . . . . . . . . . . . . . . . . 23

4.3 Continuum limit . . . . . . . . . . . . . . . . . 27

4.4 Numerical calculations . . . . . . . . . . . . . . . . . . . . . 33

5 Two dimensional lattice gauge theory with fermion content . . . . . . . . . . 37

5.1 Introduction . . . . . . . . . . . . . . . . . 37

5.2 Fermions on the lattice . . . . . . . . . . . . . . . . . 38

5.3 Fermion determinant . . . . . . . . . . . . . . . . . . . . . . 42

5.4 Algorithms for numerical calculation . . . . . . . . . . . . . 54

6 Observables and results from numerical calculations . . . . . . . . . . 57

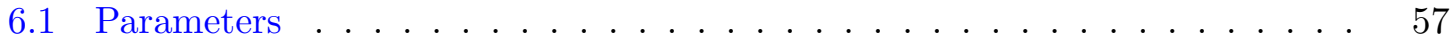

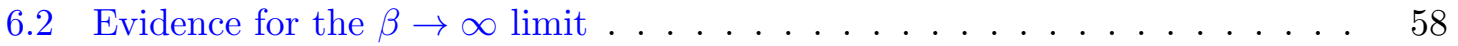

6.3 Approach to the infinite spatial extent . . . . . . . . . . . 59

6.4 Extraction of the continuum limit . . . . . . . . . . . 74

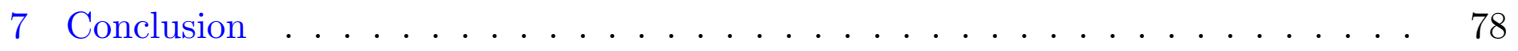

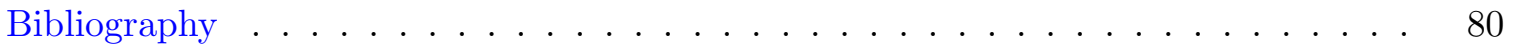

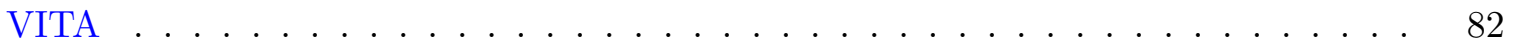




\section{LIST OF FIGURES}

FIGURE

PAGE

2.1 Gauge field configuration $\ldots \ldots \ldots \ldots$

3.1 Gauge field configuration . . . . . . . . . . . . . . . . . 14

3.2 Largest Wilson loop . . . . . . . . . . . . . . . . . . . . 15

4.1 Lattice regions to facilitate Wilson loop calculation . . . . . . . . . . . 20

4.2 A plot of free energy $e^{-F}$ as a function of area $\ln (A / 2) \ldots \ldots 34$

4.3 A plot of free energy $e^{-F}$ as a function of area $A \ldots \ldots$

5.1 Gauge field configuration . . . . . . . . . . . . . . 37

$5.2 \quad$ Fundamental plaquettes . . . . . . . . . . . . . . . . . . . 38

6.1 A plot of $m_{M}\left(m_{q}\right)$ as a function of $\beta$ at $L=4$ and $b=0.25$ at six different sample values of $m_{q} \ldots \ldots \ldots \ldots \ldots$

6.2 A plot of $m_{M}\left(m_{q}\right)$ as a function of $\beta$ at $L=6$ and $b=0.25$ at six different sample values of $m_{q} \ldots \ldots \ldots \ldots \ldots$

6.3 A plot of $m_{M}\left(m_{q}\right)$ as a function of $\beta$ at $L=4$ and $b=0.5$ at six different sample values of $m_{q}$

6.4 A plot of $m_{M}\left(m_{q}\right)$ as a function of $\beta$ at $L=6$ and $b=0.5$ at six different sample values of $m_{q} \ldots \ldots \ldots \ldots \ldots \ldots$

6.5 A plot of $m_{B}\left(m_{q}\right)$ as a function of $\beta$ at $L=4$ and $b=0.25$ at six different sample values of $m_{q} \ldots \ldots \ldots \ldots \ldots \ldots$

6.6 A plot of $m_{B}\left(m_{q}\right)$ as a function of $\beta$ at $L=6$ and $b=0.25$ at six different sample values of $m_{q} \ldots \ldots \ldots \ldots$

6.7 A plot of $m_{B}\left(m_{q}\right)$ as a function of $\beta$ at $L=4$ and $b=0.5$ at six different sample values of $m_{q}$

6.8 A plot of $m_{B}\left(m_{q}\right)$ as a function of $\beta$ at $L=6$ and $b=0.5$ at six different sample values of $m_{q}$. 
6.9 A plot of $m_{M}\left(m_{q}\right)$ as a function of $m_{q}$ at $b=0.25$ for $L=4,5,6 \ldots \quad \ldots 8$

6.10 A plot of $m_{M}\left(m_{q}\right)$ as a function of $m_{q}$ at $b=0.375$ for $L=4,5,6 \ldots \ldots 9$

6.11 A plot of $m_{M}\left(m_{q}\right)$ as a function of $m_{q}$ at $b=0.5$ for $L=4,5,6 \ldots \ldots 70$

6.12 A plot of $m_{B}\left(m_{q}\right)$ as a function of $m_{q}$ at $b=0.25$ for $L=4,5,6 \ldots \ldots$. . 71

6.13 A plot of $m_{B}\left(m_{q}\right)$ as a function of $m_{q}$ at $b=0.375$ for $L=4,5,6 \ldots \ldots 72$

6.14 A plot of $m_{B}\left(m_{q}\right)$ as a function of $m_{q}$ at $b=0.5$ for $L=4,5,6 \ldots \ldots 73$

6.15 A plot of $m_{M}\left(m_{q}\right)$ as a function of $m_{q}$ at $L=6$ for $b=0.25,0.375,0.5 . \quad \ldots \quad 76$

6.16 A plot of $m_{B}\left(m_{q}\right)$ as a function of $m_{q}$ at $L=6$ for $b=0.25,0.375,0.5$. . $\quad 77$ 


\section{CHAPTER 1}

\section{Motivations}

Quantum Chromodynamics (QCD) is the study of relativistic quantum field theory which deals with the strong interaction between fundamental particles, namely quarks and gluons. These make up nuclear matter in the universe [1-4]. There are six flavors of quarks (up, down, strange, charm, top and bottom). Each one of these flavors interact with the same non-abelian gauge field background. The non-abelian gauge field is known as the color field. From experimental evidence, it has been found that the associated non-abelian group is the special unitary group, $S U(N)$ with $N=3$. Moreover, each flavor of quark is assigned $N(=3)$ color degrees of freedom and there are $\left(N^{2}-1\right)(=8)$ types of gluon fields that make up the single non-abelian gauge field. The study of the strong interaction distinguishing it from other interactions viz: electromagnetic and gravitational interaction is usually referred as the study of Quantum Chromodynamics. There are several fundamental features of the theory:

1. There are no free parameters [2-4].

2. Scale invariance of the theory present at the classical level is broken upon quantization $[2-4]$

3. Chiral symmetry of the theory present at the classical level is broken upon quantization $[2-4]$.

4. Quarks and gluons are confined at low temperatures and low densities resulting in baryonic matter (protons, neutrons, etc) and mesonic matter (pions, etc) [2-4]

5. There is a deconfinement temperature which is currently under experimental verification above which there is quark and gluonic matter [5].

6. There is a critical density of matter (probably close to the density present inside neutron stars) above which chiral symmetry is expected to be restored [6].

Much of the phenomena listed above are not accessible in perturbation theory because of strong nature of the interaction. A widely used non-perturbative technique is Lattice Gauge Theory [7-9]. Two dimensional (one space and one time) non-abelian gauge theory coupled 
to fermions serves as an interesting toy model that shares several features with the four dimensional physically relevant theory. Studies in the direction of non-abelian gauge theory in two dimensions were pioneered by 't Hooft $[10,11]$. Our research also goes in non-abelian gauge theory in two dimensions with the aim of studying the physical properties of two dimensional Quantum Chromodynamics. The physics of confinement is usually investigated using Wilson loops [7-9] whereas the deconfinement transition is studied by using Polyakov loops $[8,9]$. Wilson loops are associated with a current loop that encloses a finite surface. The net color flux passing through this surface is proportional to the area of the surface in a confining theory, and the coefficient of the area term is the string tension. Polyakov loops are current loops that wind around the entire periodic space and do not enclose a surface. The Polyakov loops act as an order parameter that separates the confined phase from the deconfined phase at finite temperature. Unlike the four dimensional theory, there is no two dimensional analogue of deconfinement transition. Therefore, the Polyakov loops themselves will not reveal any new physics insights but correlations of Polyakov loops will reveal some new physics insights. A new aspect studied in this thesis is the correlation of two Polyakov loops oriented in two different directions.

The Hamiltonian formalism of a field theory can be converted to a path integral formalism following Feynman. The Euclidean version of the path integral can be studied in close analogy with stastical mechanics. [12-15]. The path integral formalism is an essential part of the lattice formulation of relativistic quantum field theories $[8,9]$. The partition function of the theory without any fermionic content can be explicitly computed $[16,17]$ and the result is a simple function of the physical area of the torus and certain invariants of the group, $S U(N)$, called Casimirs. We will carry this one step further and compute the correlation of Polyakov loops in two different directions. The computed correlation of Polyakov loops is only a function of the representations of two Polyakov loops and the size of the torus. The expectation value of the correlation of Polyakov loops in two different directions gives information about the energy needed to produce such a state out of the vacuum. If for some specific choice of the two Polyakov loops, the energy turns out to be 
negative, such a state would be favored as the vacuum state of the theory. The primary aim of this research is to provide an answer to the vacuum structure.

We will return to the Hamlitonian formalism of the two dimensional gauge theory to study the spectrum of physical particles in two dimensional gauge theory. Since the theory is confined, the physical particles, like in four dimensional QCD, are mesons and baryons. The Hamiltonian formalism developed as part of this thesis will enable us to extract the lightest meson and baryon. We will extract the masses as a function of the quark masses at different spatial extents and different lattice spacings. Using this information, we will be able to extract the spectrum at infinite spatial extent and the continuum. Thesis will provide an answer to the question of the mass gap in QCD - the lightest masses when the quark mass is set to zero. In addtion, it will also extract the leading dependence on quark mass. 


\section{CHAPTER 2}

\section{Basics of gauge theory on the lattice}

\subsection{Introduction}

The Hamiltonian associated with a Relativistic Quantum Field Theory (QFT) contains all the information needed to obtain the full particle spectrum of the theory. That is to say, one can formally write down the eigenvalue problem of the Hamiltonian:

$$
H|n\rangle=E_{n}|n\rangle ; \quad n=0,1,2, \cdots .
$$

where $E_{n}$ is the energy associated with the state $|n\rangle$ which contains all the information about the required quantum numbers. Let us assume that the states are ordered such that $0<E_{0}<E_{1}<E_{2}<\cdots$. The finite temperature partition function is

$$
Z(\beta)=\operatorname{Tr} e^{-\beta H}=\sum_{n} e^{-\beta E_{n}} .
$$

where $\beta$ is the inverse temperature. We will interpret the finite temperature as a Euclidean time direction for the sake of computing observables in the following sense: Let $\beta$ be divided into two intervals; $\tau$ and $\beta-\tau$ with $0<\tau<\beta$. Let

$$
\psi=\sum_{n=0}^{\infty} a_{n}|0\rangle\langle n|
$$

denote the creation of a specific state from the vacuum. The propagation of this state over an Euclidean time $\tau$ at a given $\beta$ is

$$
G_{\psi}(\tau, \beta)=\frac{1}{Z(\beta)} \operatorname{Tr} \psi e^{-\tau H} \psi^{\dagger} e^{-(\beta-\tau) H}
$$

In the zero temperature limit, $\beta \rightarrow \infty$, the propagation of this state simplifies to

$$
G_{\psi}(\tau, \infty)=\sum_{n=0}^{\infty} a_{n} a_{n}^{*} e^{-\tau\left(E_{n}-E_{0}\right)} .
$$


The large Euclidean time behavior will be dominated by the lowest energy state $E_{k}$ for which $a_{k} a_{k}^{*} \neq 0$. Therefore, by carefully choosing states, $\psi$, one can obtain information above the energy eigenstates by studying propagation at large Euclidean time at zero temperature. Using the standard Feynman Path Integral [18] one can convert the above expressions to involve a $d$-dimensional Euclidean action.

Focusing on the problem at hand for this thesis, the gauge invariant Euclidean YangMills action in d-dimensions is given as

$$
S[A]=\frac{1}{4 g^{2}} \int d^{d} x \sum \operatorname{Tr} F_{\mu \nu} F_{\mu \nu}
$$

where,

$A_{\mu}(x)$ : Non-abelian Gluon Field and an element of the underlying Lie Algebra governing the non-abelian group

$F_{\mu \nu}=\partial_{\mu} A_{\nu}-\partial_{\nu} A_{\mu}+i\left[A_{\mu}, A_{\nu}\right]:$ Field Tensor where the first two terms are the usual Abelian part and the commutator in the third term arises from the non-abelian nature.

$g$ : gauge coupling.

A local gauge transformation takes

$$
A_{\mu}(x) \rightarrow-i g(x) \partial_{\mu} g^{-1}(x)+g(x) A_{\mu}(x) g^{-1}(x)
$$

with $g(x)$ being an element of the non-abelian group. Under this transformation, the field tensor transforms covariantly:

$$
F_{\mu \nu}(x) \rightarrow g(x) F_{\mu \nu}(x) g^{-1}(x)
$$

and therefore the action is gauge invariant.

Since the action is a dimensionless quantity, dimensional analysis of the quantities in (2.6) shows that the dimension of $g$ is $[M]^{-d+4}$. Hence, $g$ is dimensionless in 4-dimensions and has positive mass dimensions for $d<4$. Therefore, theories are physically interesting only in $d \leq 4$ because of renormalizability constraints [4]. 
The Euclidean parititon function is

$$
Z=\int D A e^{\left(-S_{Y M}(A)\right)} .
$$

The integral is over all gluon fields and one needs to properly define the limits such that one obtains finite results. The procedure to get finite result is referred to as regularization and we will use a lattice for this purpose.

To obtain the Yang-Mills action on a d-dimensional lattice, we introduce a d-dimensional hyper-cubic lattice $\Lambda$ with lattice spacing $a$ as

$$
\Lambda=\left[\mathbf{n}=(\mathbf{x}, k)=\left(x_{1}, x_{2} \ldots x_{d-1}, k\right)\right] .
$$

Where, $x_{i}=1,2, \ldots ., L_{i}$ with $i=1,2,3 \ldots d-1$ and $k=1,2, \ldots, L_{d}$. We will assume periodic boundary conditions in all $d$ directions in order to reduce finite volume effects in space and realize the finite temperature partition function. The elements $n$ are called sites and the line connecting two neighboring sites are called links. The gauge fields, $A_{\mu}(x)$, in the continuum at the location $x$ and the direction $\mu$ are associated with parallel transporters on links by

$$
U_{\mu}(n)=\exp \left[i A_{\mu}(n)\right]
$$

The Wilson action in terms of elementary closed loops called plaquettes is given by

$$
S_{G}[U]=\frac{\beta}{N} \sum_{n \in \Lambda} \sum_{\mu<\nu} \operatorname{Re}\left[\operatorname{Tr}\left[1-U_{\mu \nu}(n)\right]\right]
$$

with $\beta=\frac{2 N}{g^{2}}$ and

$$
U_{\mu \nu}(n)=U_{\mu}(n) U_{\nu}(n+\hat{\mu}) U_{\mu}^{\dagger}(n+\hat{\nu}) U_{\nu}^{\dagger}(n)
$$


is the elementary plaquette at site $n$. The elementary plaquette reduces to the continuum action in the limit of small gauge fields. Furthermore, under a gauge transformation,

$$
U_{\mu}(n) \rightarrow g(n) U_{\mu}(n) g^{-1}(n+\hat{\mu})
$$

we see that the elementary plaquette transforms convariantly:

$$
U_{\mu \nu}(n) \rightarrow g(n) U_{\mu \nu}(n) g^{-1}(n)
$$

and the Wilson action is gauge invariant.

The partition function on our finite periodic lattice is

$$
Z=\int D[U] e^{-S_{G}[U]}
$$

which is a finite and well defined quantity. Insertion of states to compute propagator corresponds to the computation of the expectation value

$$
<O>=\frac{1}{Z} \int D[U] e^{-S_{G}[U]} O[U]
$$

where $O(U)$ is the operator of interest that creates the appropriate state at some time and destroys it a time, $\tau$, later. Since the action is gauge invariant, it follows that $O(U)$ has to be gauge invariant for it to be a physical observable.

\subsection{Wilson loops and Polyakov loops}

It follows from the gauge transformation property of $U_{\mu}(n)$ that the parallel transporter associated with any closed loop on the lattice will transform covariantly. Therefore, its trace will be gauge invariant. We define the trace of a product of link variables along a closed loop as

$$
L[U]=\operatorname{Tr}\left[\prod_{(n, \mu) \in L} U_{\mu}(n)\right] .
$$


Here $L$ is a closed loop of links on the lattice and the product in (2.18) runs over all these links in the order in which they appear in the loop. There are two types of loops on a periodic lattice: loops that can be smoothly deformed to a point and loops that wind around the periodic lattice. The first type is usually referred to as Wilson loops and the second type is referred to as Polyakov loops. Wilson loops enclose an area and therefore their expectation value is related to the flux through that loop. If the theory confines, the flux will be proportional to the area and result in a string tension. If the theory does not confine, the flux is usually proportional to the perimeter like in electromagnetism. Polyakov loops do not enclose an area but can be used as an order parameter to study the transition from a confined phase to a deconfined phase.

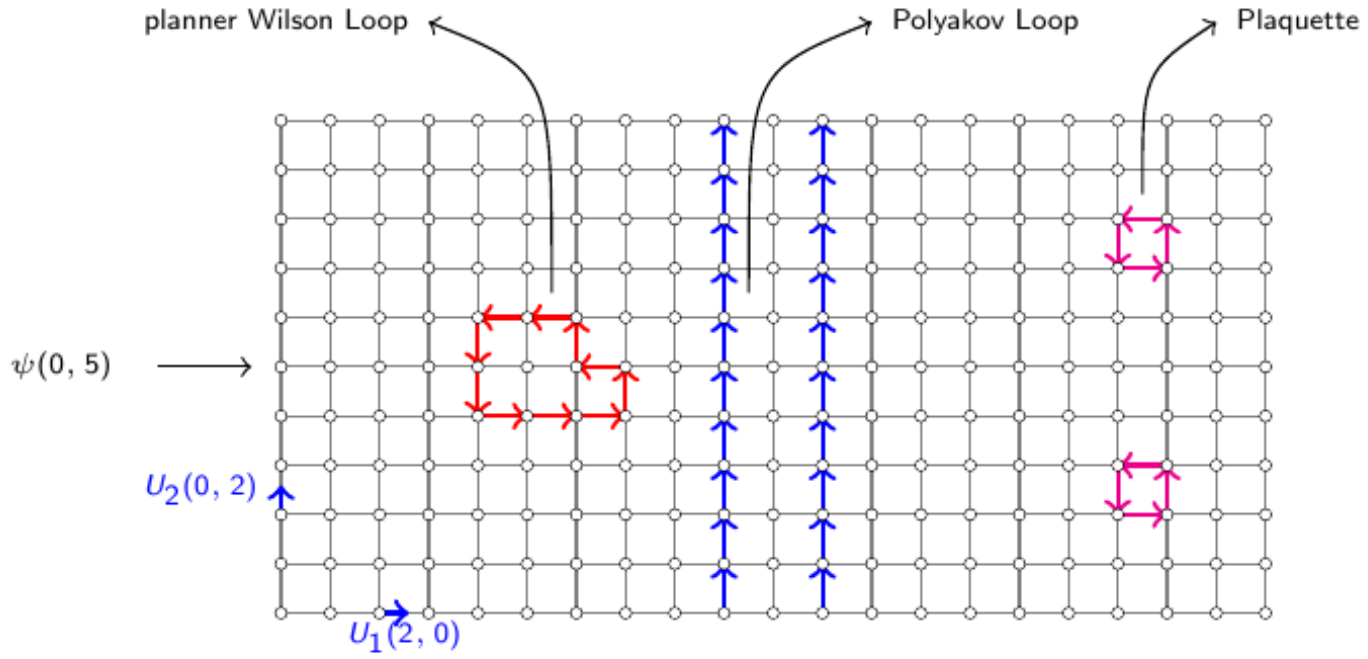

Figure 2.1: Gauge field configuration

Consider a rectangular Wilson loop with spatial extent, $R$, and Euclidean time extent, $T$. We can view this as creating a state from vacuum at some time, propagating the state for a Euclidean time, $T$ and then destroying the state. The state itself has a linear extent of $R$ and can be thought of a string of length $R$ since it is the product of parallel transporters along a spatial line. For large $T$, we expect

$$
\langle W(R, T)\rangle \propto e^{-T V(R)}
$$


The potential energy associated with the string of length $R$ is $V(R)$. In a theory like electromagnetism, we expect $V(R) \propto \frac{1}{R}$. In a confining theory, $V(R) \propto R$ and it behaves like a string with a non-zero tension.

Thus from the large- $T$ behavior of the Wilson loop, we can calculate the static quarkantiquark potential where the correction terms are exponentially suppressed. There is indefinite linear rise in the potential as the charges are separated which signals the confinement. That is to say, for large $R, V(R) \sim \sigma R$, where $\sigma$ is called the string tension. From above equations, the leading term is

$$
-\ln (\langle W(R, T)\rangle) \sim \sigma T R
$$

and is the 'area law' [9] for large $T$ and $R$. In a nonconfining theory, the potential energy should become a constant at large distance. In that case the leading term has the form

$$
-\ln (\langle W(R, T)\rangle) \sim e_{0}(T+R)
$$

which is a 'perimeter law' [9] for large T and R.

Consider a gauge group of the form $S U(N)$. Such a group has a non-trivial center called $Z_{N}$. In addition to local gauge transformations, the Wilson action is also invariant under a global transformation of the form:

$$
U_{\mu}(n) \rightarrow e^{i \frac{2 \pi k_{\mu}}{N}} U_{\mu}(n) ; \quad k_{\mu} \in[0, N-1]
$$

since the center of the group commutes with all elements and leaves Wilson loops invariant. But, it does transform the Polakov loop and therefore the expectation value of the Polyakov loop is zero. If the center of the group is spontaneously broken, we will see the Polyakov loop behave as an order parameter and this is referred to as the measure of the deconfining transition. 


\subsection{Continuum limit}

The Yang-Mills functional integral (2.12) contains a single parameter ' $g$ ' called the bare gauge coupling. The continuum limit is obtained by taking the number of lattice points, $L \rightarrow \infty$, and varying $g(L)$ such that physical observables remain invariant. It is not a priori clear that this will happen in practice. If it does happen, we refer to the theory as "renormalizable" and the Yang-Mills theory is an example of such a theory. Furthermore, it is known to be an asymptotically free theory, implying that $g \rightarrow 0$ as $L \rightarrow \infty$. It is customary to define, $a=\frac{1}{L}$ and refer to $a \rightarrow 0$ as the continuum limit.

Let $P(g(a), a)$ be a physical observable which in the limit $a \rightarrow 0$ obtains its physical value $P_{0}$. Callan and Symanzik [19-21], following early suggestions for QED by Stuckelberg, Peterman, GellMann, and Low $[22,23]$ formulated the requirement of constant physics in a differential equation

$$
\frac{d P(g(a), a)}{\ln a}=\left(\frac{\partial}{\partial \ln a}+\frac{\partial g}{\partial \ln a} \frac{\partial}{\partial g}\right) P(g, a)=0
$$

Here the coefficient function of the second term is called the $\beta$-function,

$$
\beta(g) \equiv-\frac{\partial g}{\partial \ln a}
$$

Above equation determines how the coupling $g$ depends on $a$ along with an integration constant. Using perturbation theory, we can expand the $\beta$-function in a power series around $g=0$. For $S U(N)$ and $n_{f}$ massless quarks

$$
\begin{aligned}
& \beta(g)=-\beta_{0} g^{3}-\beta_{1} g^{5}+\mathcal{O}\left(g^{7}\right), \\
& \beta_{0}=\frac{1}{(4 \pi)^{2}}\left(\frac{11}{3} N-\frac{2}{3} n_{f}\right), \\
& \beta_{1}=\frac{1}{(4 \pi)^{4}}\left(\frac{34}{3} N^{2}-\frac{10}{3} N n_{f}-\frac{N^{2}-1}{N} n_{f}\right) .
\end{aligned}
$$

One can show that the first two coefficients of the expansion are universal and independent of the regularization scheme. In general, however, the $\beta$-function depends on the details of 
the regularization. Keeping only the first two terms, the differential Eq. (2.24) with (2.25) can be solved using separation of variables to get

$$
a(g)=\frac{1}{\Lambda_{L}}\left(\beta_{0} g^{2}\right)^{-\frac{\beta_{1}}{2 \beta_{0}^{2}}} \exp \left(-\frac{1}{2 \beta_{0} g^{2}}\right)\left(1+\mathcal{O}\left(g^{2}\right)\right) .
$$

The integration constant $\Lambda_{L}$ comes automatically to set the scale by fixing the value of $g$ at some $a$. Inverting the relation (2.26), we can obtain the coupling $g$ as a function of $a$ which is called running coupling,

$$
g(a)^{-2}=\beta_{0} \ln \left(a^{-2} \Lambda_{L}^{-2}\right)+\frac{\beta_{1}}{\beta_{0}} \ln \left(\ln \left(a^{-2} \Lambda_{L}^{-2}\right)\right)+\mathcal{O}\left(1 / \ln \left(a^{2} \Lambda_{L}^{2}\right)\right) .
$$

Therefore a change in $a$ induces a corresponding change in $g$ such that physical observables remain independent of the scale-fixing procedure. The value of $\Lambda_{L}$ (the subscript $L$ refers to the lattice regularization). Different regularizations will result in different $\Lambda$. 


\section{CHAPTER 3}

\section{Two dimensional lattice gauge theory without fermion content}

\subsection{Introduction}

Two dimensional non-abelian gauge theories are particularly simple to study but reveal a wealth of physics insights. Migdal [16] studied this theory in the context of recursion equations since these equations become exact in two dimensions. Gross and Taylor [17] showed that the partition function of two dimensional QCD is a string theory. Gross and Witten [24] started from the lattice theory with the standard Wilson action on an infinite lattice and showed factorization to independent plaquettes prompting a possible connection between infinite volume gauge theories and matrix model in a certain limit [25]. Unlike the four dimensional theory, this theory is always in the confined phase.

In chapter two we study the behavior of Wilson loops and Polyakov loops in SU(N) gauge theory on a two dimensional torus $[8,9]$. A certain gauge fixing form referred as the maximal tree [26] is used to rewrite the action in terms of elementary plaquette (flux on elementary lattice squares) variables and two Polyakov loop variables. There is also a constraint imposed by the theory being defined on a two dimensional torus.

In the absence of fermions, the partition function, the expectation value of Wilson loops and the expectation value of Polyakov loop correlations can be computed analytically. Because of translational invariance, correlation between Polyakov loops in two different directions is only a function of the size of the torus and the representation carried by the Polyakov loop. Focussing on $\mathrm{SU}(2)$, we use numerical diagonalization to compute the energy eigenvalues associated with the insertion of Polyakov loops in two different directions. We

will show that there is one particular insertion that lowers the energy compared to the vacuum and therefore the true vacuum contains the insertion of this operator [27]. 


\subsection{Gauge field configuration}

Consider a $L_{1} \times L_{2}$ periodic lattice where one dimension is Euclidean time and another dimension is space. There are $\left(2 L_{1} L_{2}\right) S U(N)$ link variables which are denoted by $U_{\mu}^{g}\left(n_{1}, n_{2}\right)$ with $0 \leq n_{1}<L_{1}$ and $0 \leq n_{2}<L_{2}$ where $\mu=1,2$. These link variables obey the periodic boundary conditions,

$$
U_{1}^{g}\left(n_{1}, L_{2}\right)=U_{1}^{g}\left(n_{1}, 0\right) ; U_{2}^{g}\left(L_{1}, n_{2}\right)=U_{2}^{g}\left(0, n_{2}\right) ; 0 \leq n_{1}<L_{1} ; 0 \leq n_{2}<L_{2} .
$$

The above gauge field configuration is obtained from one representative gauge field configuration, $U_{\mu}\left(n_{1}, n_{2}\right)$, by a gauge transformation:

$$
\begin{aligned}
& U_{1}^{g}\left(n_{1}, n_{2}\right)=g^{\dagger}\left(n_{1}, n_{2}\right) U_{1}\left(n_{1}, n_{2}\right) g\left(n_{1}+1, n_{2}\right) ; \\
& U_{2}^{g}\left(n_{1}, n_{2}\right)=g^{\dagger}\left(n_{1}, n_{2}\right) U_{2}\left(n_{1}, n_{2}\right) g\left(n_{1}, n_{2}+1\right) .
\end{aligned}
$$

where $g\left(n_{1}, n_{2}\right)$ is a periodic function defined on the lattice sites. A choice for the representative guage field configuration is:

- $U_{1}\left(n_{1}, n_{2}\right)=1$; for $0 \leq n_{1}<L_{1}-1$ and $0 \leq n_{2}<L_{2}$

- $U_{2}\left(0, n_{2}\right)=1$; for $0 \leq n_{2}<L_{2}-1$

- $U_{1}\left(L_{1}-1,0\right)=T_{1}$;

- $U_{2}\left(0, L_{2}-1\right)=T_{2}$;

- $U_{2}\left(n_{1}+1, n_{2}\right)=U_{p}\left(n_{1}, n_{2}\right) U_{2}\left(n_{1}, n_{2}\right)$; for $0 \leq n_{1}<L_{1}-1$; and $0 \leq n_{2}<L_{2}$.

- $U_{1}\left(L_{1}-1, n_{2}+1\right)=U_{2}^{\dagger}\left(L_{1}-1, n_{2}\right) U_{p}^{\dagger}\left(L_{1}-1, n_{2}\right) U_{1}\left(L_{1}-1, n_{2}\right)$; for $0 \leq n_{2}<L_{2}-1$. 


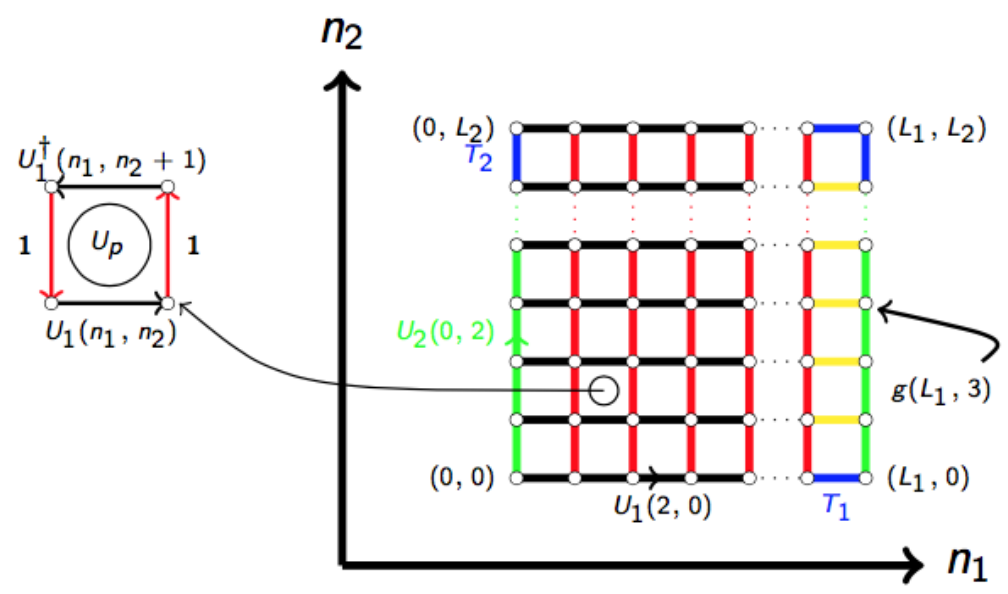

Figure 3.1: Gauge field configuration

The integration over all $2 L_{1} L_{2}$ number of $U_{\mu}^{g}$ variables can be split into

- $\left(\left(L_{1} L_{2}\right)-1\right) U_{p}\left(n_{1}, n_{2}\right)$ variables for all $0 \leq n_{1}<L_{1} ; 0 \leq n_{2}<L_{2}$ except $\left(n_{1}, n_{2}\right)=$ $\left(L_{1}-1, L_{2}-1\right)$;

- $T_{1}$ and $T_{2}$;

- $\left(\left(L_{1} L_{2}\right)-1\right) g\left(n_{1}, n_{2}\right)$ variables for all $0 \leq n_{1}<L_{1} ; 0 \leq n_{2}<L_{2}$ except $\left(n_{1}, n_{2}\right)=$ $\left(L_{1}-1, L_{2}-1\right)$; which is set to identity.

Consider the largest Wilson operator $W=T_{1} T_{2} T_{1}^{\dagger} T_{2}^{\dagger}$. This operator can be expressed as the product of all the plaquette operators inside the Wilson loop as:

$$
W=T_{1} T_{2} T_{1}^{\dagger} T_{2}^{\dagger}=\prod_{n_{2}=L_{2}-1}^{0} \prod_{n_{1}=L_{1}-1}^{0} U_{p}\left(n_{1}, n_{2}\right)
$$

Such that

$$
\left[T_{2} T_{1} T_{2}^{\dagger} T_{1}^{\dagger}\right]\left[\prod_{n_{2}=L_{2}-1}^{0} \prod_{n_{1}=L_{1}-1}^{0} U_{p}\left(n_{1}, n_{2}\right)\right]=1
$$




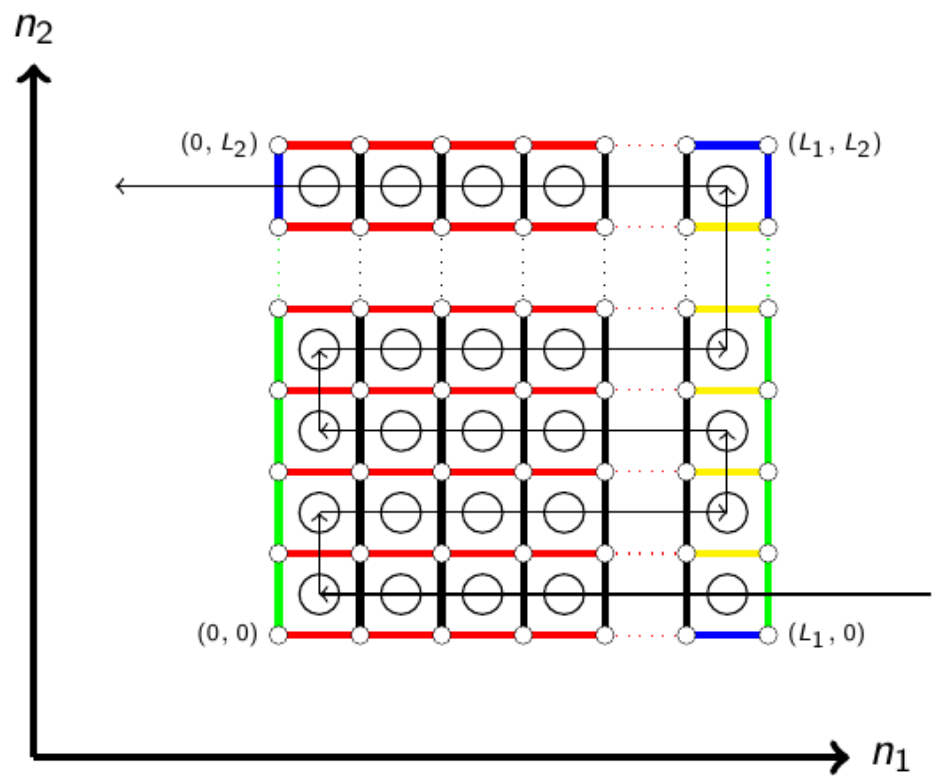

Figure 3.2: Largest Wilson loop

where the product is path ordered. Above equation is a constraint on the plaquette variable, $U_{p}\left(L_{1}-1, L_{2}-1\right)$ which is not present in the set of integration variables.

We take the action as a function of single plaquette variables and is dependent on the coupling constant $\beta$;

$$
e^{S_{g}}=\prod_{n_{1}=0}^{L_{1}-1} \prod_{n_{2}=0}^{L_{2}-1} f_{p}\left[U_{p}\left(n_{1}, n_{2}\right) ; \beta\right] .
$$

where $f_{p}$ is a class function. The characters (traces) in the different irreducible representation form a basis for a class function and therefore,

$$
f_{p}\left[U_{p}\left(n_{1}, n_{2}\right) ; \beta\right]=\sum_{r} \tilde{\beta}_{r}(\beta) \chi_{r}\left(U_{p}\left(n_{1}, n_{2}\right)\right)
$$

The class function is real and therefore the coefficients in the character expansion have to obey $\tilde{\beta}_{r}(\beta)=\tilde{\beta}_{\bar{r}}(\beta)$.ie. $\tilde{\beta}_{r}(\beta)$ is real. 
While taking continuum limit, we keep physical coupling $g^{2}$ fixed and we set $\beta$ by taking lattice spacing $a \rightarrow 0$ as;

$$
\beta=\frac{1}{g^{2} a^{2}}
$$

We keep the size of the torus fixed as we take the continuum limit by setting dimensionless area

$$
A=\frac{L_{1} L_{2}}{\beta}=\left(a L_{1}\right)\left(a L_{2}\right) g^{2}
$$

fixed as we take $a \rightarrow 0$ and $\left(L_{1} L_{2}\right) \rightarrow \infty$.

\subsection{Partition function}

We use all $L_{1} L_{2}$ plaquettes to define our partition function by keeping the restriction as in (3.4) on integral over $T_{1}$ and $T_{2}$. The finite volume partition function is defined as

$$
Z\left(\beta ; L_{1} L_{2}\right)=\prod_{n_{1}=0}^{L_{1}-1} \prod_{n_{2}=0}^{L_{2}-1} \int\left[d U_{p}\left(n_{1}, n_{2}\right)\right] f_{p}\left[U_{p}\left(n_{1}, n_{2}\right) ; \beta\right] \int d T_{1} d T_{2} \delta\left[W\left(L_{1} L_{2}\right), T_{1} T_{2} T_{1}^{\dagger} T_{2}^{\dagger}\right] .
$$

To express the partition function in more convenient form, first we consider the integral involving variables $T_{1}$ and $T_{2}$ :

$$
I\left(T_{1} T_{2}\right)=\int d T_{1} d T_{2} \delta\left[W\left(L_{1} L_{2}\right), T_{1} T_{2} T_{1}^{\dagger} T_{2}^{\dagger}\right]
$$

We use the completeness relation [Hamermesh:1962gp]

$$
\delta(U, V)=\sum_{r \alpha \beta} d_{r} D_{\alpha \beta}^{r}(U) D_{\beta \alpha}^{r}\left(V^{\dagger}\right)=\sum_{r} d_{r} \operatorname{Tr}\left(U V^{\dagger}\right),
$$


which is a delta function defined on the group with $U, V \in S U(N)$. The sum runs over all representations, r, with $D_{\alpha \beta}^{r}$ being representation of $U$ labeled $r$ and $d_{r}$ being the dimension of that representation. In addtion, we use orthogonality relation [28]

$$
\int D U D_{\alpha \beta}^{r}(U) D_{\gamma \delta}^{s}\left(U^{\dagger}\right)=\delta_{r s} \frac{\delta_{\alpha \delta} \delta_{\beta \gamma}}{d_{r}}
$$

to get

$$
\int d T_{1} d T_{2} \delta\left[W, T_{1} T_{2} T_{1}^{\dagger} T_{2}^{\dagger}\right]=\sum_{r} \frac{1}{d_{r}} \chi_{r}(W)
$$

Using the result in (3.13), the partition function reduces to the form

$$
Z\left(\beta ; L_{1} L_{2}\right)=\prod_{n_{1}=0}^{L_{1}-1} \prod_{n_{2}=0}^{L_{2}-1} \int\left[d U_{p}\left(n_{1}, n_{2}\right)\right] f_{p}\left[U_{p}\left(n_{1}, n_{2}\right) ; \beta\right]\left[\sum_{r} \frac{1}{d_{r}} \chi_{r}\left(W\left(L_{1}, L_{2}\right)\right)\right]
$$

Now we are left with integrals independent of $T_{1}$ and $T_{2}$. By simple rearrangements we get it as

$$
Z\left(\beta ; L_{1} L_{2}\right)=\sum_{r} \frac{1}{d_{r}} \prod_{n_{1}=0}^{L_{1}-1} \prod_{n_{2}=0}^{L_{2}-1} \int\left[d U_{p}\left(n_{1}, n_{2}\right)\right] f_{p}\left[U_{p}\left(n_{1}, n_{2}\right) ; \beta\right] \chi_{r}\left(W\left(L_{1}, L_{2}\right)\right) .
$$

Using the identity that follows from (3.12),

$$
\int D U \chi_{s}(U) \chi_{r}\left(V U^{\dagger} W\right)=\delta_{s r} \frac{\chi_{r}(V W)}{d_{r}}
$$

we can integrate out all $U_{p}\left(n_{1}, n_{2}\right)$, one after another, to obtain

$$
Z\left(\beta ; L_{1} L_{2}\right)=\sum_{r}\left[\frac{\tilde{\beta}_{r}(\beta)}{d_{r}}\right]^{L_{1} L_{2}}=\left[\tilde{\beta}_{0}(\beta)\right]^{L_{1} L_{2}} \sum_{r}\left[\frac{\tilde{\beta}_{r}(\beta)}{d_{r} \tilde{\beta}_{0}(\beta)}\right]^{L_{1} L_{2}}
$$


The final expression for the partition function has a simple interpretation. A simple choice for $\tilde{\beta}_{r}(\beta)$ is based on the heat kernel action:

$$
\tilde{\beta}_{r}(\beta)=d_{r} e^{-\frac{C_{r}^{(2)}}{N \beta}} ; \quad \tilde{\beta}_{0}(\beta)=1
$$

where $C_{r}^{(2)}$ is the quadratic Casimir in the $r$ representation. Inserting this into the expression for the partition function results in

$$
Z\left(\beta ; L_{1} L_{2}\right)=\sum_{r} e^{-\frac{C_{r}^{(2)} L_{1} L_{2}}{N \beta}} .
$$

Since $\frac{L_{1} L_{2}}{\beta}$ is the physical area, we see that the partition function is a function of the area with different representations weighted according to the Casimirs. In the next section, we will obtain expressions for Wilson loops and correlations of Polyakov loops in order to extract physical informattion about the pure gauge theory. 


\section{CHAPTER 4}

\section{Observables and results from analytical calculations}

\subsection{Wilson loops}

Consider a $K_{1} \times K_{2}$ rectangular loop with corners at $(0,0),\left(K_{1}-1,0\right),\left(0, K_{2}-1\right)$ and $\left(K_{1}-1, K_{2}-1\right)$ and with $0<K_{1} \leq L_{1}-1$ and $0<K_{2} \leq L_{2}-1$. As in the case of the physical size of the torus defined in (3.8), to keep the size of the loop fixed as we take the continuum limit, we define a dimensionless area of the loop

$$
X=\frac{K_{1} K_{2}}{\beta}=\left(a K_{1}\right)\left(a K_{2}\right) g^{2}
$$

The area $X$ is fixed when $a \rightarrow 0$ and $\left(L_{1} L_{2}\right) \rightarrow \infty$. The Wilson loop operator is given by

$$
W\left(K_{1}, K_{2}\right)=\prod_{i_{2}=0}^{K_{2}-1}\left[\prod_{i_{1}=K_{1}-1}^{0} U_{p}\left(i_{1}, i_{2}\right)\right] .
$$

Starting from (3.15), the expectation value for Wilson Loop can be expressed as

$$
\begin{aligned}
& \left\langle\frac{\chi_{r}\left(W\left(K_{1} K_{2}\right)\right)}{d_{r}}\right\rangle \\
& =\frac{\prod_{n_{1}=0}^{L_{1}-1} \prod_{n_{2}=0}^{L_{2}-1} \int\left[d U_{p}\left(n_{1}, n_{2}\right)\right] f_{p}\left[U_{p}\left(n_{1}, n_{2}\right) ; \beta\right]\left[\sum_{r^{\prime}} \frac{1}{d_{r^{\prime}}} \chi_{r^{\prime}}\left(W\left(L_{1}, L_{2}\right)\right)\right] \frac{\chi_{r}\left(W\left(K_{1} K_{2}\right)\right)}{d_{r}}}{Z\left(\beta ; L_{1} L_{2}\right)}
\end{aligned}
$$

As in the case of the partition function, we can use (3.16) and integrate out all $U_{p}\left(n_{1}, n_{2}\right)$ which does not appear in $W\left(K_{1}, K_{2}\right)$. For this we separate the whole region into four different parts: Region -I,II,III, and IV. Only plaquettes from region - I appear in $W\left(K_{1}, K_{2}\right)$. We calculate the expectation value in following steps: 


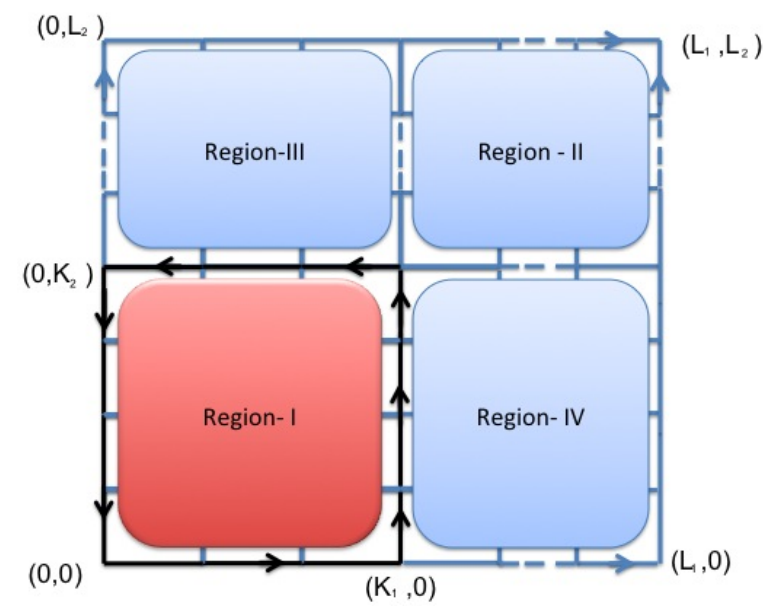

Figure 4.1: Lattice regions to facilitate Wilson loop calculation

$$
\begin{aligned}
& \left\langle\frac{\chi_{r}\left(W\left(K_{1} K_{2}\right)\right)}{d_{r}}\right\rangle
\end{aligned}
$$

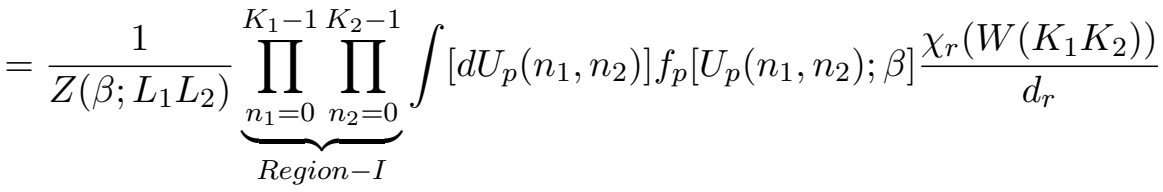

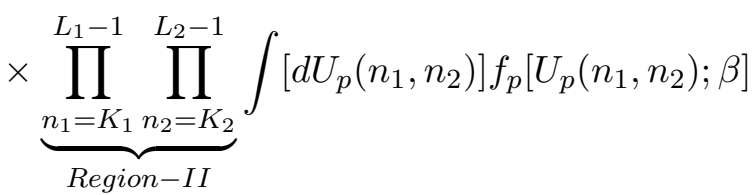

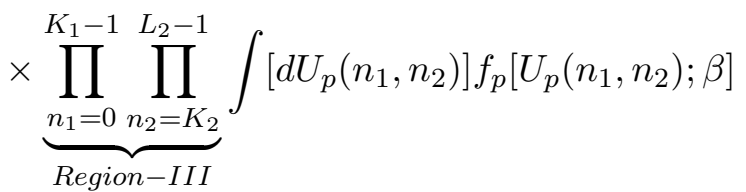

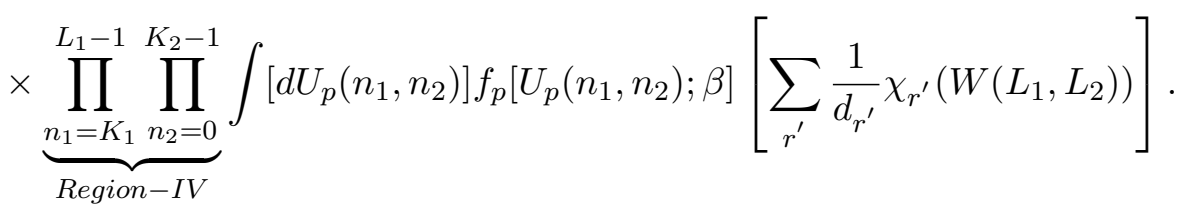


We start with last term corresponding to region -IV with notation $W\left(L_{1}, L_{2}\right)=$ $W(I, I I, I I I, I V)$

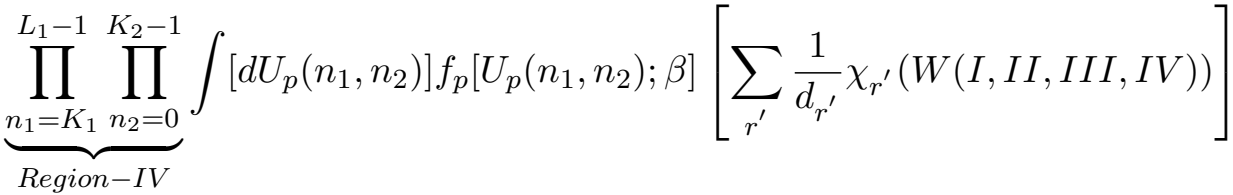

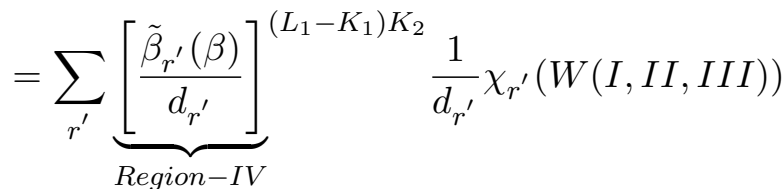

Inserting the value obtained from last integral (region-IV) to second last integral (region-III) we obtain:

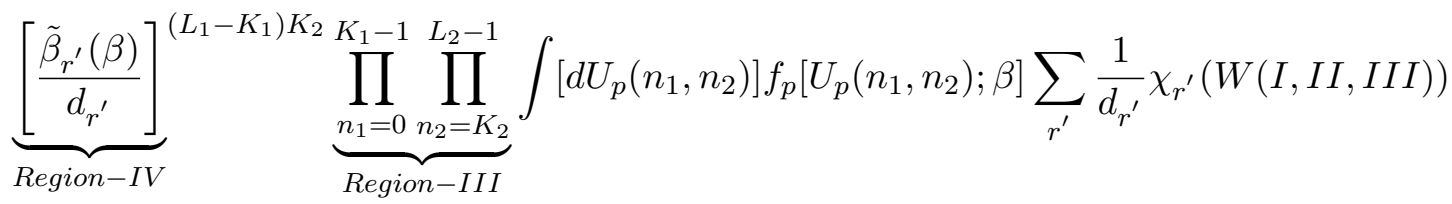

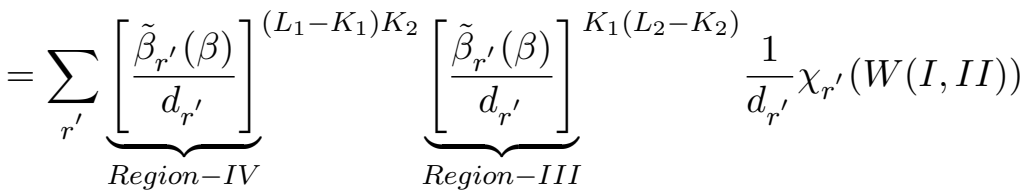

Inserting the value obtained from second last integral (region-III) to second integral (regionII) we obtain:

$$
\begin{aligned}
& \underbrace{\left[\frac{\tilde{\beta}_{r^{\prime}}(\beta)}{d_{r^{\prime}}}\right]}_{\text {Region }-I V, I I I} \underbrace{\left(L_{1}-K_{1}\right) K_{2}+K_{1}\left(L_{2}-K_{2}\right)}_{\text {Region-II }} \underbrace{L_{1}-1}_{\prod_{1}=K_{1}} \prod_{n_{2}=K_{2}}^{L_{2}-1} \int\left[d U_{p}\left(n_{1}, n_{2}\right)\right] f_{p}\left[U_{p}\left(n_{1}, n_{2}\right) ; \beta\right] \sum_{r^{\prime}} \frac{1}{d_{r^{\prime}}} \chi_{r^{\prime}}(W(I, I I)) \\
& =\sum_{r^{\prime}} \underbrace{\left[\frac{\tilde{\beta}_{r^{\prime}}(\beta)}{d_{r^{\prime}}}\right]}_{\text {Region-IV,III,II }} \underbrace{\left[\left(L_{1}-K_{1}\right) K_{2}+K_{1}\left(L_{2}-K_{2}\right)+\left(L_{1}-K_{1}\right)\left(L_{2}-K_{2}\right)\right]}_{\left(L_{1} L_{2}-K_{1} K_{2}\right)} \frac{1}{d_{r^{\prime}}} \chi_{r^{\prime}}(W(I))
\end{aligned}
$$

Where $W(I)=W\left(K_{1}, K_{2}\right)$. 
Inserting the value obtained from second integral (region-II) to first integral (region-I) we obtain an intermediate expression

$$
\begin{aligned}
& \left\langle\frac{\chi_{r}\left(W\left(K_{1} K_{2}\right)\right)}{d_{r}}\right\rangle=\sum_{r^{\prime}} \underbrace{\left[\frac{\tilde{\beta}_{r^{\prime}}(\beta)}{d_{r^{\prime}}}\right]}_{\text {Region }-I V, I I I, I I} \times \frac{1}{Z\left(\beta ; L_{1} L_{2}\right)} \\
& \times \underbrace{\prod_{n_{1}=0}^{K_{1}-1} \prod_{n_{2}=0}^{K_{2}-1} \int\left[L_{1} L_{2}-K_{1} K_{2}\right)}_{\text {Region }-I}\left[d U_{p}\left(n_{1}, n_{2}\right)\right] f_{p}\left[U_{p}\left(n_{1}, n_{2}\right) ; \beta\right] \frac{1}{d_{r^{\prime}}} \chi_{r^{\prime}}\left(W\left(K_{1}, K_{2}\right)\right) \frac{1}{d_{r}} \chi_{r}\left(W\left(K_{1} K_{2}\right)\right)
\end{aligned}
$$

We use $f_{p}\left[U_{p}\left(n_{1}, n_{2}\right) ; \beta\right]$ from (3.6) and perform integration for first region using the Clebsch-Gordon series

$$
\chi_{r}(U) \chi_{r^{\prime}}(U)=\sum_{r^{\prime \prime}} n\left(r, r^{\prime} ; r^{\prime \prime}\right) \chi_{r^{\prime \prime}}(U)
$$

where $n\left(r, r^{\prime} ; r^{\prime \prime}\right)$ is the number of times the representation, $r^{\prime \prime}$, appears in the tensor product $r \otimes r^{\prime}$. Finally, we arrive at

$$
\begin{aligned}
& \left\langle\frac{\chi_{r}\left(W\left(K_{1} K_{2}\right)\right)}{d_{r}}\right\rangle=\sum_{r^{\prime}} \underbrace{\left[\frac{\tilde{\beta}_{r^{\prime}}(\beta)}{d_{r^{\prime}}}\right]}_{\text {Region-IV,III,II }} \times \frac{1}{Z\left(\beta ; L_{1} L_{2}\right)} \\
& \times \underbrace{\times}_{\sum_{r^{\prime \prime}} \frac{\tilde{\beta}_{r}(\beta)}{d_{r} d_{r^{\prime}}} \sum_{r^{\prime \prime}} n\left(r, r^{\prime} ; r^{\prime \prime}\right) \underbrace{\prod_{r_{1}=0}^{n_{1}=0} \prod_{n_{2}=0}^{K_{1}-1}\left[\frac{K_{2}-1}{K_{r^{\prime \prime}}}\left[\begin{array}{c}
\tilde{\beta}^{\prime \prime}(\beta) \\
d_{r^{\prime \prime}}
\end{array}\right]^{\left(K_{1} K_{2}\right)}\right.}_{d_{r} r_{r^{\prime}}}\left[d U_{p}\left(n_{1}, n_{2}\right)\right] \chi_{r^{\prime \prime}}(U) \chi_{r}\left(W\left(K_{1} K_{2}\right)\right)} \\
& =\frac{\sum_{r^{\prime}}\left[\frac{\tilde{\beta}_{r^{\prime}}(\beta)}{d_{r^{\prime}}}\right]^{\left(L_{1} L_{2}-K_{1} K_{2}\right)} \sum_{r^{\prime \prime}} \frac{n\left(r, r^{\prime} ; r^{\prime \prime}\right) d_{r^{\prime \prime}}}{d_{r} d_{r^{\prime}}^{\prime}}\left[\frac{\tilde{\beta}_{r^{\prime \prime}}(\beta)}{d_{r^{\prime \prime}}}\right]^{\left(K_{1} K_{2}\right)}}{\sum_{r^{\prime}}\left[\frac{\tilde{\beta}_{r^{\prime}}(\beta)}{d_{r^{\prime}}}\right]^{L_{2} L_{2}}} .
\end{aligned}
$$


Using (3.17) we can write

$$
\left\langle\frac{\chi_{r}\left(W\left(K_{1} K_{2}\right)\right)}{d_{r}}\right\rangle=\frac{\sum_{r^{\prime}}\left[\frac{\tilde{\beta}_{r^{\prime}}(\beta)}{d_{r^{\prime}} \tilde{\beta}_{0}(\beta)}\right]^{\left(L_{1} L_{2}-K_{1} K_{2}\right)} \sum_{r^{\prime \prime}} \frac{n\left(r, r^{\prime} ; r^{\prime \prime}\right) d_{r^{\prime \prime}}}{d_{r} d_{r^{\prime}}}\left[\frac{\tilde{\beta}_{r^{\prime \prime}(\beta)}(\beta)}{d_{r^{\prime \prime}} \tilde{\beta}_{0}(\beta)}\right]^{\left(K_{1} K_{2}\right)}}{\sum_{r^{\prime}}\left[\frac{\tilde{\beta}_{r^{\prime}}(\beta)}{d_{r^{\prime}} \tilde{\beta}_{0}(\beta)}\right]^{L_{2} L_{2}}}
$$

One can proceed further and compute the correlations of multiple Wilson loops where no two loops have a single plaquette in common and show that the correlations do not depend on the separation. The indepenence of seperation is a consequence of the form of the partition function in (3.9) where all plaquettes are independent except for a global constraint that only depends on the area.

\subsection{Polyakov loops}

In order to consider the correlation between Polyakov loops oriented in different directions, we start from (3.9) and consider expectation values of the form

$$
\begin{aligned}
& \left\langle\chi_{r_{1}}\left(T_{1}\right) \chi_{r_{2}}\left(T_{2}\right)\right\rangle=\frac{1}{Z\left(\beta ; L_{1} L_{2}\right)} \prod_{n_{1}=0}^{L_{1}-1} \prod_{n_{2}=0}^{L_{2}-1} \int\left[d U_{p}\left(n_{1}, n_{2}\right)\right] f_{p}\left[U_{p}\left(n_{1}, n_{2}\right) ; \beta\right] \\
& \times \int d T_{1} d T_{2} \underbrace{\delta\left[W\left(L_{1}, L_{2}\right), T_{1} T_{2} T_{1}^{\dagger} T_{2}^{\dagger}\right]}_{\sum_{r} d_{r} \chi_{r}\left[W\left(L_{1}, L_{2}\right) T_{2} T_{1} T_{2}^{\dagger} T_{1}^{\dagger}\right]} \chi_{r_{1}}\left(T_{1}\right) \chi_{r_{2}}\left(T_{2}\right) .
\end{aligned}
$$

As before, we use the definition of $\delta$ - function:

$$
\delta(U, V)=\sum_{r} d_{r} \chi_{r}\left(U, V^{\dagger}\right)
$$


Using delta function we get intermediate expression

$$
\begin{aligned}
& \left\langle\chi_{r_{1}}\left(T_{1}\right) \chi_{r_{2}}\left(T_{2}\right)\right\rangle=\frac{1}{Z\left(\beta ; L_{1} L_{2}\right)} \prod_{n_{1}=0}^{L_{1}-1} \prod_{n_{2}=0}^{L_{2}-1} \int\left[d U_{p}\left(n_{1}, n_{2}\right)\right] f_{p}\left[U_{p}\left(n_{1}, n_{2}\right) ; \beta\right] \\
& \times \int d T_{1} d T_{2}\left\{\sum_{r} d_{r} \chi_{r}\left[W\left(L_{1}, L_{2}\right) T_{2} T_{1} T_{2}^{\dagger} T_{1}^{\dagger}\right]\right\} \chi_{r_{1}}\left(T_{1}\right) \chi_{r_{2}}\left(T_{2}\right) .
\end{aligned}
$$

First, we perform all $U_{p}\left(n_{1}, n_{2}\right)$ integrals. Integral (4.14) can be written as:

$$
\begin{aligned}
& \left\langle\chi_{r_{1}}\left(T_{1}\right) \chi_{r_{2}}\left(T_{2}\right)\right\rangle=\frac{1}{Z\left(\beta ; L_{1} L_{2}\right)} \int d T_{1} d T_{2} \chi_{r_{1}}\left(T_{1}\right) \chi_{r_{2}}\left(T_{2}\right) \\
& \times \prod_{n_{1}=0}^{L_{1}-1} \prod_{n_{2}=0}^{L_{2}-1} \int\left[d U_{p}\left(n_{1}, n_{2}\right)\right] f_{p}\left[U_{p}\left(n_{1}, n_{2}\right) ; \beta\right]\left\{\sum_{r} d_{r} \chi_{r}\left[W\left(L_{1}, L_{2}\right) T_{2} T_{1} T_{2}^{\dagger} T_{1}^{\dagger}\right]\right\} .
\end{aligned}
$$

Using $f_{p}\left[U_{p}\left(n_{1}, n_{2}\right) ; \beta\right]$ from (3.6) and using (4.13), we get

$$
\begin{aligned}
& \left\langle\chi_{r_{1}}\left(T_{1}\right) \chi_{r_{2}}\left(T_{2}\right)\right\rangle \\
& =\frac{1}{Z\left(\beta ; L_{1} L_{2}\right)} \sum_{r} d_{r}\left[\frac{\tilde{\beta}_{r}(\beta)}{d_{r}}\right]^{L_{1} L_{2}} \int d T_{1} d T_{2} \chi_{r_{1}}\left(T_{1}\right) \chi_{r_{2}}\left(T_{2}\right) \chi_{r}\left(T_{2} T_{1} T_{2}^{\dagger} T_{1}^{\dagger}\right) .
\end{aligned}
$$

Next, consider the integral:

$$
I\left(T_{1}, T_{2}\right)=\int d T_{1} d T_{2} \chi_{r_{1}}\left(T_{1}\right) \chi_{r_{2}}\left(T_{2}\right) \chi_{r}\left(T_{2} T_{1} T_{2}^{\dagger} T_{1}^{\dagger}\right)
$$

Inserting the explicit expressions, $\chi_{r}\left(T_{2} T_{1} T_{2}^{\dagger} T_{1}^{\dagger}\right)=\sum_{k l m n} D^{r}\left(T_{2}\right)_{k l} D^{r}\left(T_{1}\right)_{l m} D^{r}\left(T_{2}^{\dagger}\right)_{m n} D^{r}\left(T_{1}^{\dagger}\right)_{n k}$, $\chi_{r_{1}}\left(T_{1}\right)=\sum_{i} D^{r_{1}}\left(T_{1}\right)_{i i}$ and $\chi_{r_{2}}\left(T_{2}\right)=\sum_{j} D^{r_{2}}\left(T_{2}\right)_{j j}$ into the integral above and separating $T_{1}$ and $T_{2}$ variables one can get

$$
I\left(T_{1}, T_{2}\right)=\sum_{k l m n i j} \underbrace{\int d T_{1} D^{r}\left(T_{1}\right)_{l m} D^{r}\left(T_{1}^{\dagger}\right)_{n k} D^{r_{1}}\left(T_{1}\right)_{i i}}_{I\left(T_{1}\right)} \underbrace{\int d T_{2} D^{r}\left(T_{2}\right)_{k l} D^{r}\left(T_{2}^{\dagger}\right)_{m n} D^{r_{2}}\left(T_{2}\right)_{j j}}_{I\left(T_{2}\right)}
$$


Let us denote

$$
\begin{gathered}
I\left(T_{1}\right)=\int d T_{1} \underbrace{D^{r}\left(T_{1}\right)_{l m} D^{r_{1}}\left(T_{1}\right)_{i i}}_{\text {matrix element of- } D^{r}\left(T_{1}\right) \otimes D^{r_{1}}\left(T_{1}\right)} D^{r}\left(T_{1}^{\dagger}\right)_{n k} ; \\
I\left(T_{2}\right)=\int d T_{2} \underbrace{D^{r}\left(T_{2}\right)_{k l} D^{r_{2}}\left(T_{2}\right)_{j j}}_{\text {matrix element of- } D^{r}\left(T_{2}\right) \otimes D^{r_{2}}\left(T_{2}\right)} D^{r}\left(T_{2}^{\dagger}\right)_{m n} .
\end{gathered}
$$

We can decompose the direct product matrix $D^{r}\left(T_{1}\right) \otimes D^{r_{1}}\left(T_{1}\right)$ into its irreducible representation parts:

$$
D^{\mu}(g) \otimes D^{\nu}(g)=S^{-1} D(g) S
$$

where,

$$
D(g)=\bigoplus_{\lambda} \alpha_{\lambda} D^{\lambda}(g)
$$

$\alpha_{\lambda}$ counts the number of times a particular representation matrix $D^{\lambda}(g)$ repeats in the block diagonal form of $D(g)$.

To get the relation for matrix element level, we express the elements of matrix $\mathrm{S}$ as the CG -coefficient of transformation. Let $\mid l, i>$ be the basis for direct product matrix $D^{r}\left(T_{1}\right) \otimes D^{r_{1}}\left(T_{1}\right)$ and $\mid \alpha\left(r, r_{1}\right), \lambda, p>$ be the basis for invariant subspaces with respect to representation matrix $D(g): \lambda=$ label of irreducible representation and $\alpha=1, \ldots, a_{\lambda}$. Then transformation from basis $\mid l, i>$ to $\mid \alpha\left(r, r_{1}\right), \lambda, p>$ can be expressed as follows:

$$
\left|\alpha\left(r, r_{1}\right), \lambda, p>=\sum_{l, i}\right| l, i>\underbrace{<l, i \mid \alpha\left(r, r_{1}\right), \lambda, p>}_{\text {CG-coff }} .
$$

Thus equation (4.20) for $T_{1}$ and $T_{2}$ can be expressed as:

$$
\begin{gathered}
D^{r}\left(T_{1}\right)_{l m} D^{r_{1}}\left(T_{1}\right)_{i i}=\sum_{\lambda, p, q}<l, i\left|\alpha\left(r, r_{1}\right), \lambda, p>D^{\lambda}\left(T_{1}\right)_{p q}<\alpha\left(r, r_{1}\right), \lambda, q\right| m i> \\
D^{r}\left(T_{2}\right)_{k l} D^{r_{2}}\left(T_{2}\right)_{j j}=\sum_{s, u, v}<k, j\left|\beta\left(r, r_{2}\right), s, u>D^{s}\left(T_{2}\right)_{u v}<\beta\left(r, r_{2}\right), s, v\right| l, j>.
\end{gathered}
$$


The expression of $I\left(T_{1}\right)$ and $I\left(T_{2}\right)$ becomes:

$$
\begin{gathered}
I\left(T_{1}\right)=\sum_{\lambda, p, q}<l, i\left|\alpha\left(r, r_{1}\right), \lambda, p><\alpha\left(r, r_{1}\right), \lambda, q\right| m i>\int d T_{1} D^{\lambda}\left(T_{1}\right)_{p q} D^{r}\left(T_{1}^{\dagger}\right)_{n k} \\
I\left(T_{2}\right)=\sum_{s, u, v}<k, j\left|\beta\left(r, r_{2}\right), s, u><\beta\left(r, r_{2}\right), s, v\right| l, j>\int d T_{2} D^{s}\left(T_{2}\right)_{u v} D^{r}\left(T_{2}^{\dagger}\right)_{m n} .
\end{gathered}
$$

using orthogonality relation: $\int d T_{1} D^{\lambda}\left(T_{1}\right)_{p q} D^{r}\left(T_{1}^{\dagger}\right)_{n k}=\frac{\delta_{\lambda r}}{d_{\lambda}} \delta_{p k} \delta_{q n}$ and $\int d T_{2} D^{s}\left(T_{2}\right)_{u v} D^{r}\left(T_{2}^{\dagger}\right)_{m n}=$ $\frac{\delta_{s r}}{d_{s}} \delta_{u n} \delta_{v m}$, and putting back the expression of $I\left(T_{1}\right)$ and $I\left(T_{2}\right)$ in (4.18),

$$
\begin{array}{r}
\left.I\left(T_{1} T_{2}\right)\right)=a\left(r_{1}, r_{2} ; r\right)=\sum_{k l m n i j} \frac{1}{d_{r} d_{r}}<l, i\left|\alpha\left(r, r_{1}\right), \lambda, k><\alpha\left(r, r_{1}\right), \lambda, n\right| m i> \\
\times<k, j\left|\beta\left(r, r_{2}\right), s, n><\beta\left(r, r_{2}\right), s, m\right| l, j>.
\end{array}
$$

Clearly this $a\left(r_{1}, r_{2} ; r\right)$ is a real number because by using the unitarity property of CGcofficients we obtain

$$
<l, i\left|\alpha\left(r, r_{1}\right), \lambda, k>=<l, i\right| \alpha\left(r, r_{1}\right), \lambda, k>^{*} \text {. }
$$

Finally we arrive at

$$
\left\langle\chi_{r_{1}}\left(T_{1}\right) \chi_{r_{2}}\left(T_{2}\right)\right\rangle=\frac{1}{Z\left(\beta ; L_{1} L_{2}\right)} \sum_{r} d_{r}\left[\frac{\tilde{\beta}_{r}(\beta)}{d_{r}}\right]^{L_{1} L_{2}} a\left(r_{1}, r_{2} ; r\right)
$$

Using (3.17), the correlation between two Polyakov loops is

$$
M_{r_{1} r_{2}}(A)=\left\langle\chi_{r_{1}}\left(T_{1}\right) \chi_{r_{2}}\left(T_{2}\right)\right\rangle=\frac{\sum_{r} d_{r} a\left(r_{1}, r_{2} ; r\right)\left[\frac{\tilde{\beta}_{r}(\beta)}{d_{r} \tilde{\beta}_{0}(\beta)}\right]^{L_{1} L_{2}}}{\sum_{r}\left[\frac{\tilde{\beta}_{r}(\beta)}{d_{r} \tilde{\beta}_{0}(\beta)}\right]^{L_{1} L_{2}}}
$$

where

$$
\begin{array}{r}
a\left(r_{1}, r_{2} ; r\right)=\sum_{k l m n i j} \frac{1}{d_{r} d_{r}}<l, i\left|\alpha\left(r, r_{1}\right), \lambda, k><\alpha\left(r, r_{1}\right), \lambda, n\right| m, i> \\
\times<k, j\left|\beta\left(r, r_{2}\right), s, n><\beta\left(r, r_{2}\right), s, m\right| l, j>
\end{array}
$$


Clearly for fixed $r$ the value of $a\left(r_{1}, r_{2} ; r\right)$ is symmetric w.r.t. label $r_{1}$ and $r_{2}$. Since for adjoint representations, these coefficients are given as $a\left(r_{1}, r_{2} ; \bar{r}\right)$. We can prove that

$$
a\left(r_{1}, r_{2} ; \bar{r}\right)=a\left(r_{1}, r_{2} ; r\right)
$$

because our representation is unitary and therefore $M(A)$ is a real symmetric matrix.

\subsection{Continuum limit}

In order to take the continuum limit, we need to take a specific lattice action. Since the continuum limit will not depend on the specific choice as long as it satisfies some essential properties, the simplest choice is the heat kernel action given by [29]. In this section we will investigate continuum limit of partition function (3.17), Wilson loop (4.11) and Polyakov loop (4.28) respectively.

\subsubsection{Continuum limit of partition function}

Heat kernel action [29] is given by

$$
\tilde{\beta}_{r}(\beta)=d_{r} e^{-\frac{C_{r}^{2}}{N \beta}}
$$

where $C_{r}^{2}$ is the quadratic Casmir in the $r$ representation. The continuum limit of the partition function $(3.17)$ is

$$
Z(A)=\sum_{r} e^{-\frac{1}{N} C_{r}^{(2)} A}
$$




\subsubsection{Continuum limit of Wilson Loop}

Similarly the continuum limit of expectation value of Wilson loop with dimensionaless area $X$ is

$$
\frac{1}{d_{r}}<\chi_{r}(W(X, A))>=\frac{\sum_{r^{\prime}, r^{\prime \prime}} \frac{n\left(r, r^{\prime} ; r^{\prime \prime}\right) d_{r^{\prime \prime}}}{d_{r} d_{r^{\prime}}} e^{-\frac{1}{N} C_{r^{\prime}}^{(2)}(A-X)-\frac{1}{N} C_{r^{\prime \prime}}^{(2)} X}}{\sum_{q} e^{-\frac{1}{N} C_{q}^{(2)} A}} .
$$

We can study few special cases using (4.33).

- Case - I : When $A \rightarrow \infty$

When $A \rightarrow \infty$, exponential terms in numerator on (4.33) can be expanded w.r.t $r^{\prime}$,

$$
\begin{aligned}
& \sum_{r^{\prime}, r^{\prime \prime}} \frac{n\left(r, r^{\prime} ; r^{\prime \prime}\right) d_{r^{\prime \prime}}}{d_{r} d_{r^{\prime}}} e^{-\frac{1}{N} C_{r^{\prime}}^{(2)}(A-X)} e^{-\frac{1}{N} C_{r^{\prime \prime}}^{(2)} X} \\
& =\sum_{r^{\prime \prime}} \frac{n\left(r, 0 ; r^{\prime \prime}\right) d_{r^{\prime \prime}}}{d_{r}} e^{-\frac{1}{N} C_{r^{\prime \prime}}^{(2)} X}+\underbrace{\sum_{r^{\prime} \neq 0, r^{\prime \prime}} \frac{n\left(r, r^{\prime} ; r^{\prime \prime}\right) d_{r^{\prime \prime}}}{d_{r} d_{r^{\prime}}} e^{-\frac{1}{N} C_{r^{\prime}}^{(2)}(A-X)} e^{-\frac{1}{N} C_{r^{\prime \prime}}^{(2)} X}}_{=0, \text { when } A \rightarrow \infty} \\
& =\sum_{r^{\prime \prime}} \frac{n\left(r, 0 ; r^{\prime \prime}\right) d_{r^{\prime \prime}}}{d_{r}} e^{-\frac{1}{N} C_{r^{\prime \prime}}^{(2)} X} .
\end{aligned}
$$

Since $n\left(r, 0 ; r^{\prime \prime}\right)$ is number of times the representation $r^{\prime \prime}$ is repeated in direct product of $r^{\prime}=0$ (trivial representation, $d_{0}=1$ ) and $r$ representation. Obviously ,

$$
n\left(r, 0 ; r^{\prime \prime}\right)=1 ; r^{\prime \prime}=r
$$

Thus numerator becomes $e^{-\frac{1}{N} C_{r}^{(2)} X}$. Similarly denominator on (4.33) gives us

$$
e^{-\frac{1}{N} C_{q}^{(2)} A}=1+\underbrace{\sum_{q \neq 0} e^{-\frac{1}{N} C_{q}^{(2)} A}}_{=0 ; \text { when } A \rightarrow \infty} .
$$


Finally we get

$$
\frac{1}{d_{r}}\left\langle\chi_{r}(W(X, \infty))\right\rangle=e^{\frac{1}{N} C_{r}^{(2)} X} .
$$

- Case - II : When $X \rightarrow 0$

$$
\frac{1}{d_{r}}\left\langle\chi_{r}(W(0, A))\right\rangle=\sum_{r^{\prime}}\left[\sum_{r^{\prime \prime}} \frac{n\left(r, r^{\prime} ; r^{\prime \prime}\right) d_{r^{\prime \prime}}}{d_{r} d_{r^{\prime}}}\right] \frac{e^{-\frac{1}{N} C_{r^{\prime}}^{(2)} A}}{\sum_{q} e^{-\frac{1}{N} C_{q}^{(2)} A}}
$$

Since the dimension of product representation is the product of individual representations, we have

$$
\operatorname{dim}\left(r \otimes r^{\prime}\right)=\sum_{r^{\prime \prime}} n\left(r, r^{\prime} ; r^{\prime \prime}\right) d_{r^{\prime \prime}}=d_{r} d_{r^{\prime}}
$$

We get

$$
\frac{1}{d_{r}}<\chi_{r}(W(0, A))>=1
$$

- Case - III : When $X \rightarrow A$

$$
\frac{1}{d_{r}}<\chi_{r}(W(A, A))>=\frac{\sum_{r^{\prime}, r^{\prime \prime}} \frac{n\left(r, r^{\prime} ; r^{\prime \prime}\right) d_{r^{\prime \prime}}}{d_{r} d_{r^{\prime}}} e^{-\frac{1}{N} C_{r^{\prime \prime}}^{(2)} A}}{\sum_{q} e^{-\frac{1}{N} C_{q}^{(2)} A}} .
$$

- Case - IV : When $X \rightarrow \infty, A \rightarrow \infty$

We start with (4.41) and take the limit $A \rightarrow \infty$. From numerator of (4.41),

$$
\sum_{r^{\prime}} \frac{n\left(r, r^{\prime} ; 0\right)}{d_{r} d_{r^{\prime}}}+\underbrace{\sum_{r^{\prime}, r^{\prime \prime} \neq 0} \frac{n\left(r, r^{\prime} ; r^{\prime \prime}\right) d_{r^{\prime \prime}}}{d_{r} d_{r^{\prime}}} e^{-\frac{1}{N} C_{r^{\prime \prime}}^{(2)} A}}_{=0 ; \text { when } A \rightarrow \infty}
$$


Since $r^{\prime \prime}=0$, which is trivial representation with $d_{r^{\prime \prime}}=d_{0}=1$. This representation is repeated once if $r^{\prime}$ is adjoint representation of $r$.(Proved in appendix-I) ie. $r^{\prime}=\bar{r}$. So that $d_{r}=d_{r^{\prime}}$. Hence contribution form numerator becomes $\frac{1}{d_{r}^{2}}$.

From denominator of (4.41);

$$
\begin{gathered}
\sum_{q} e^{-\frac{1}{N} C_{q}^{(2)} A}=1+\underbrace{\sum_{q \neq 0} e^{-\frac{1}{N} C_{q}^{(2)} A}}_{=0 ; \text { when } A \rightarrow \infty}=1 \\
\therefore \frac{1}{d_{r}}\left\langle\chi_{r}(W(\infty, \infty))\right\rangle=\frac{1}{d_{r}^{2}}
\end{gathered}
$$

\subsubsection{Continuum limit of Polyakov loop}

The continuum limit of the correlation of Polyakov loops oriented in two different directions in a torus of dimensionless area $\mathrm{A}$ is

$$
M_{r_{1} r_{2}}(A)=<\chi_{r_{1}}\left(T_{1}(A)\right) \chi_{r_{2}}\left(T_{2}(A)\right)>=\frac{\sum_{r} d_{r} a\left(r_{1}, r_{2} ; r\right) e^{-\frac{1}{N} C_{r}^{(2)} A}}{\sum_{q} e^{-\frac{1}{N} C_{q}^{(2)} A}}
$$

- Case - I : When $A \rightarrow \infty$

In the limit of $A \rightarrow \infty$, numerator can be expanded as;

$$
\sum_{r} d_{r} a\left(r_{1}, r_{2} ; r\right) e^{-\frac{1}{N} C_{r}^{(2)} A}=d_{0} a\left(r_{1}, r_{2} ; 0\right)+\underbrace{\sum_{r} d_{r} a\left(r_{1}, r_{2} ; r\right) e^{-\frac{1}{N} C_{r}^{(2)} A}}_{=0 ; \text { when } A \rightarrow \infty}
$$

Obviously for trivial representation $d_{0}=1$ and

$$
a\left(r_{1}, r_{2} ; 0\right)=\delta_{r_{1} 0} \delta_{r_{2} 0}
$$


Contribution from numerator is $\delta_{r_{1} 0} \delta_{r_{2} 0}$ and from denominator we get;

$$
\sum_{q} e^{-\frac{1}{N} C_{q}^{(2)} A}=1+\underbrace{\sum_{q \neq 0} e^{-\frac{1}{N} C_{q}^{(2)} A}}_{=0 ; \text { when } A \rightarrow \infty}=1
$$

Therefore,

$$
M_{r_{1} r_{2}}(\infty)=\delta_{r_{1} 0} \delta_{r_{2} 0}
$$

There is only one non-zero eigenvalue for this matrix which is one and all other eigenvalues are zero.

- Case - I : When $r_{2}=0$ or $r_{1}=0$

For $r_{2}=0$ and $r_{1}=0$;

$$
M_{r_{1} 0}(A)=\frac{\sum_{r} d_{r} a\left(r_{1}, 0 ; r\right) e^{-\frac{1}{N} C_{r}^{(2)} A}}{\sum_{q} e^{-\frac{1}{N} C_{q}^{(2)} A}}
$$

\subsubsection{The special case of $\mathrm{SU}(2)$}

In order to obtain further insight into the correlation function between Polyakov loops in the two directions, we focus on the $\mathrm{SU}(2)$ group. We start with general expression of continuum limit of Polyakov loops which is a symmetric matrix $M(A)$ from equation (4.45),

$$
M_{r_{1} r_{2}}(A)=\frac{\sum_{r} d_{r} a\left(r_{1}, r_{2} ; r\right) e^{-\frac{1}{N} C_{r}^{(2)} A}}{\sum_{q} e^{-\frac{1}{N} C_{q}^{(2)} A}}
$$

Let $s, s_{1}$ and $s_{2}$ label irreducible representations of $\mathrm{SU}(2)$ matrix. Then these labels should be positive integer or an half integer. The casmir operator takes on a value of $s(s+1)$ for representation label $s$ and dimension of representation is $(2 s+1)$. Therefore

$$
M_{s_{1} s_{2}}(A)=\frac{\sum_{s}(2 s+1) a\left(s_{1}, s_{2} ; s\right) e^{-\frac{s(s+1)}{2} A}}{\sum_{s} e^{-\frac{s(s+1)}{2} A}}
$$


Where

$$
a\left(s_{1}, s_{2} ; s\right)=\int d T_{1} d T_{2} \chi_{r_{1}}\left(T_{1}\right) \chi_{r_{2}}\left(T_{2}\right) \chi_{r}\left(T_{2} T_{1} T_{2}^{\dagger} T_{1}^{\dagger}\right)
$$

To calculate these cofficients $a\left(s_{1}, s_{2} ; s\right)$, we start with $T_{1}$ as the diagonal matrix in its fundamental representation

$$
T_{1}=\left(\begin{array}{cc}
e^{i \eta} & 0 \\
0 & e^{i \eta}
\end{array}\right) ; \quad \eta_{1} \in[0, \pi]
$$

The most general form of $T_{2}$ in the fundamental representation is

$$
T_{2}=\left(\begin{array}{cc}
\cos \theta_{2} e^{i \alpha_{2}} & \sin \theta_{2} e^{i \beta_{2}} \\
-\sin \theta_{2} e^{-i \beta_{2}} & \cos \theta_{2} e^{-i \alpha_{2}}
\end{array}\right) ; \quad \theta_{2} \in\left[0, \frac{\pi}{2}\right] ; \alpha_{2}, \beta_{2} \in[0,2 \pi]
$$

Let the eigen value of $T_{2}$ is $e^{ \pm i \eta_{2}}$. Using the invariance of the trace,

$$
\cos \eta_{2}=\cos \theta_{2} \cos \alpha_{2} ; \quad \eta_{2} \in[0, \pi]
$$

The product matrix $T_{2} T_{1} T_{2}^{\dagger} T_{1}^{\dagger}$ becomes:

$\left.T_{2} T_{1} T_{2}^{\dagger} T_{1}^{\dagger}=\left(\begin{array}{cc}\cos ^{2} \theta_{2}+\sin ^{2} \theta_{2} e^{-2 i \eta_{1}} & -\sin 2 \theta_{2}\left(e^{i\left(\alpha_{2}+2 \eta_{1}+\beta_{2}\right)}-e^{i\left(\beta_{2}+\alpha_{2}\right)}\right) \\ \sin 2 \theta_{2}\left(e^{-i\left(\alpha_{2}+2 \eta_{1}+\beta_{2}\right)}-e^{-i\left(\beta_{2}+\alpha_{2}\right)}\right) & \cos ^{2} \theta_{2}+\sin ^{2} \theta_{2} e^{2 i \eta_{1}}\end{array}\right) 4.57\right)$

Let $e^{ \pm i \eta}$ be the eigen value of this marix. Then

$$
\cos \eta=\frac{1}{2}\left[2 \cos ^{2} \theta+2 \sin ^{2} \theta \cos 2 \eta\right]=1-2 \sin ^{2} \theta \sin ^{2} \eta_{1} ; \eta \in[0, \pi]
$$

For eigenvalue $e^{ \pm i \psi}$ in fundamental representation of matrix of $\mathrm{SU}(2)$ group, the character of that matrix in any irreducible representation label $j$ is given by

$$
\chi_{r}=\frac{\sin \left(j+\frac{1}{2}\right) \psi}{\sin \left(\frac{\psi}{2}\right)}
$$


From (4.53), we get

$$
\begin{aligned}
& a\left(s_{1}, s_{2} ; s\right) \\
= & \frac{2}{\pi} \int_{0}^{\pi} d \eta_{1} \sin \eta_{1} \sin \left(2 s_{1}+1\right) \eta_{1} \int_{0}^{\pi} d \theta_{2} \sin 2 \theta_{2} \frac{\sin (2 s+1) \eta}{\sin \eta} \int_{0}^{2 \pi} \frac{d \alpha}{2 \pi} \frac{\sin \left(2 s_{2}+1\right) \eta_{2}}{\sin \eta_{2}}
\end{aligned}
$$

Upon insertion of this expression into (4.52), we obtain the correlation between Polyakov loops in two different directions for the case of $\mathrm{SU}(2)$. Since loops in two different representations are correlated, we need to diagonalize, $M_{s_{1} s_{2}}(A)$ to find the eigenstates of the Polyakov loop operator. These eigenstates will be linear combinations of different representations. In order to find these eigenstates, we need to use a numerical method since we have a infinite dimensional matrix.

\subsection{Numerical calculations}

The $M_{s_{1} s_{2}}$ is a real symmetric matrix. It's eigenvalues are real and eigenvectors are orthogonal. We calculate $a\left(s_{1}, s_{2} ; s\right)$ using (4.60), and insert the value in (4.51) to get a matrix $M_{s_{1} s_{2}}$. The quantity $a\left(s_{1}, s_{2} ; s\right)$ obeys a selection rule where $s_{1}$ and $s_{2}$ must satisfy $0 \leq s_{1}, s_{2} \leq 2 s$. Thus for a value of $s \leq S$, we get $M_{s_{1} s_{2}}$ as a $(2 S+1) \times(2 S+1)$ matrix. We numerically diagonalize this finite matrix and increase $S$ till a few low lying eigenvalues have converged. The results are plotted as a function of the dimensionless area A. 


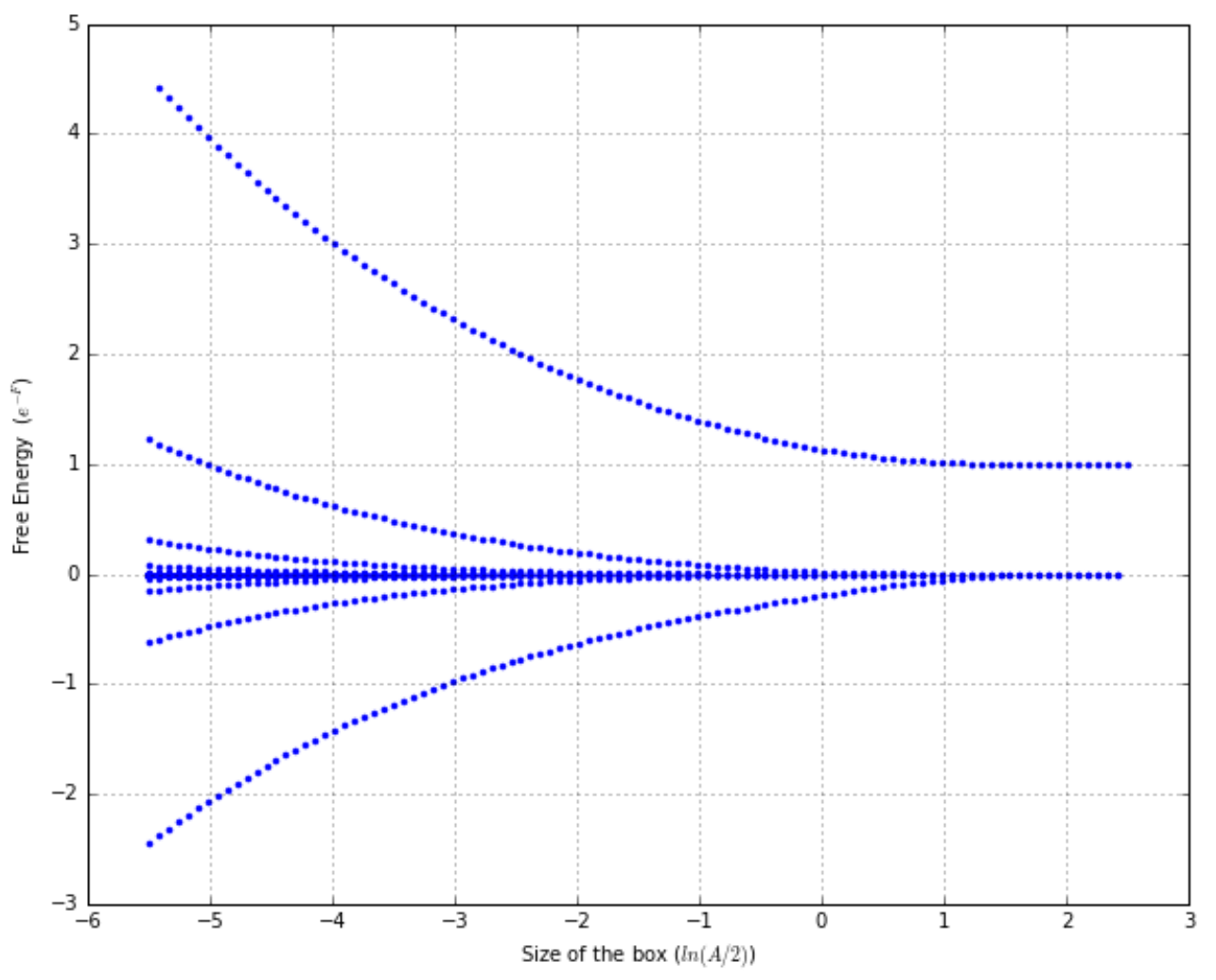

Figure 4.2: A plot of free energy $e^{-F}$ as a function of area $\ln (A / 2)$ 


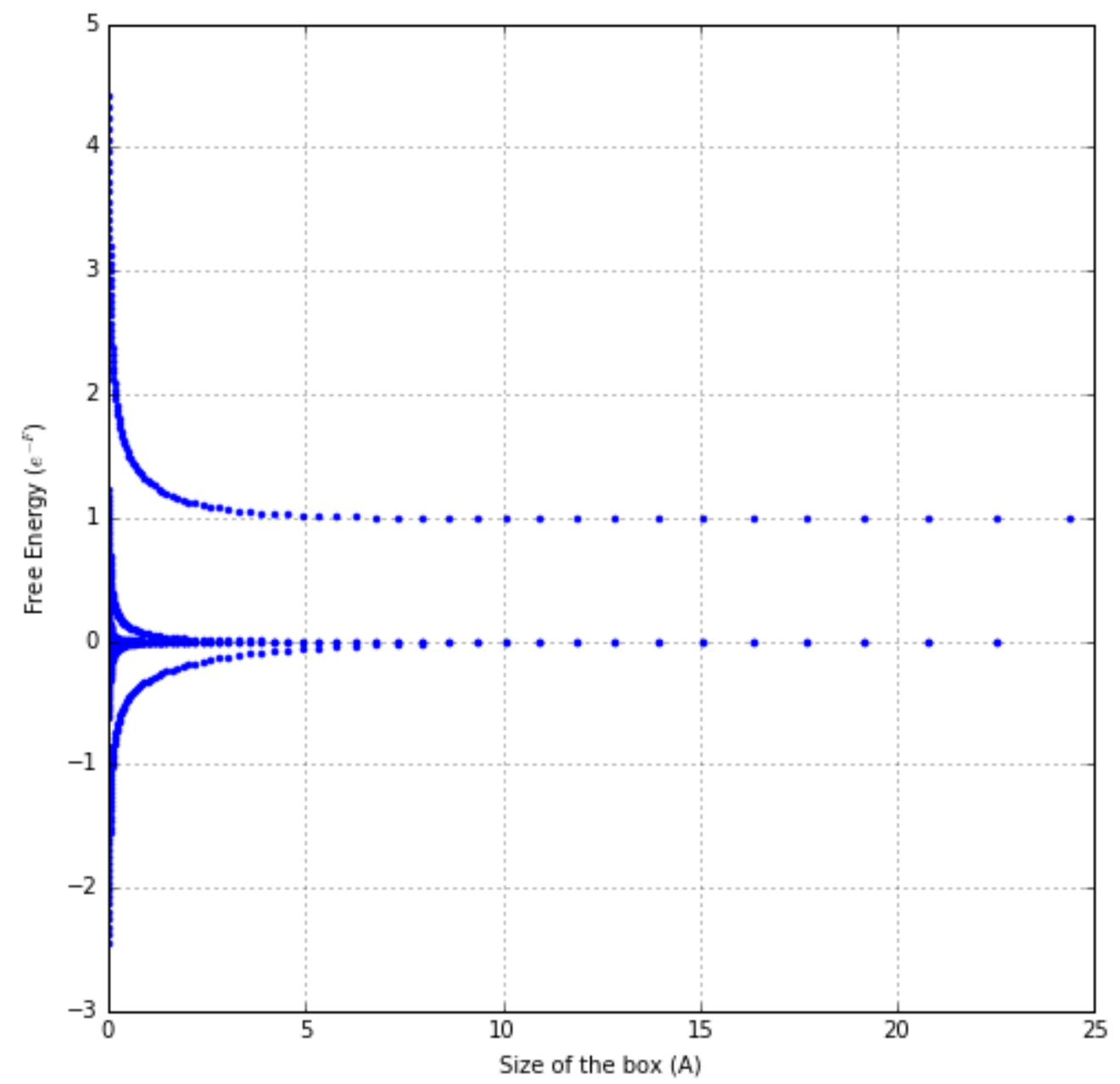

Figure 4.3: A plot of free energy $e^{-F}$ as a function of area $A$

In the large area limit, $M_{00}(\infty)=1$ and all others are zero. Property of $M$ says that insertion of Polyakov loops in any nontrivial representation costs infinite amount of energy. The matrix, $M(A)$, for $S U(2)$ at finite $A$ has every entry in the range [-1,1]. Upon diagonalization at a fixed $A$, we have new normalized eigenvectors of the form

$$
\xi_{i}(T(A))=\sum_{s} b_{i}^{s}(A) \chi_{s}(T(A)), i=0,1, \ldots
$$


where $b_{i}^{s}$ is the contribution from the reprentation $s$ to the $i^{\prime}$ th eigenvector. The corresponding eigenvalues, $\lambda_{i}(A)$, satisfy $\lambda_{i}(A)>\lambda_{i+1}(A)$. Each eigenvector, $\xi_{i}(\theta ; A)$, is an even function of $\theta \in[-\pi, \pi]$ where $e^{ \pm i \theta}$ are the eigenvalues of $T(A)$ in the fundamental representation. The eigenvectors are normalized according to

$$
\frac{2}{\pi} \int_{0}^{\pi} d \theta \sin ^{2} \theta \xi_{i}(\theta ; A) \xi_{j}(\theta ; A)=\delta_{i j}
$$

Only integer valued $s$ contribute to the sum and therefore, $\xi_{i}(\theta ; A)=\xi_{i}(\pi-\theta ; A)$.

The plot of the eigenvalues $\lambda_{i}(A)$ shown in Fig. 4.3 has two main features:

1. There is one eigenvalues, $\lambda_{i}(A)>1$, for all finite $A$ and it approaches unity as $A \rightarrow \infty$.

2. All other eigenvalues are less than $\lambda_{0}(A)$ in magnitude and approach zero as $A \rightarrow \infty$ Since the expectation value of $\xi_{0}\left(T_{1}(A)\right) \xi_{0}\left(T_{2}(A)\right)$ is greater than unity, the true vacuum of the theory contains the insertion of this operator. Viewed as a function of $\theta$, $\xi_{0}(\theta ; A)$ will develop a peak at $\theta=0$ as we decrease $A$ from infinity.

Our results show that the true vacuum of two dimensional Yang-Mills theory is not trivial when the theory is defined in a finite area. Instead it favors the presence of a specific Polyakov loop operator in each direction. We have computed the details of this Polyakov loop operator using numerical techniques for the special case of $\mathrm{SU}(2)$. 


\section{CHAPTER 5}

\section{Two dimensional lattice gauge theory with fermion content}

\subsection{Introduction}

In this chapter, two dimensional QCD will be studied with the aim of extracting meson and baryon masses with $\mathrm{SU}(3)$ gauge fields and two degenerate flavors of quarks. For this purpose, it will be convenient to use the canonical formalism. After providing the details of the canonical formalism, a numerical algorithm for the computation of the meson and baryon masses will be presented. The numerical data so obtained will be used to extract the behavior of the meson and baryon masses as a function of quark masses.

\subsubsection{Canonical formalism}

Canonical formalism involves the Hamiltonian and therefore we will work with the gauge fields in the Weyl gauge. Since we are working on a periodic lattice in both the space and Euclidean time direction, Weyl gauge corresponds to fixing all links in the time direction to unity except the links that close the periodicity in the time direction.

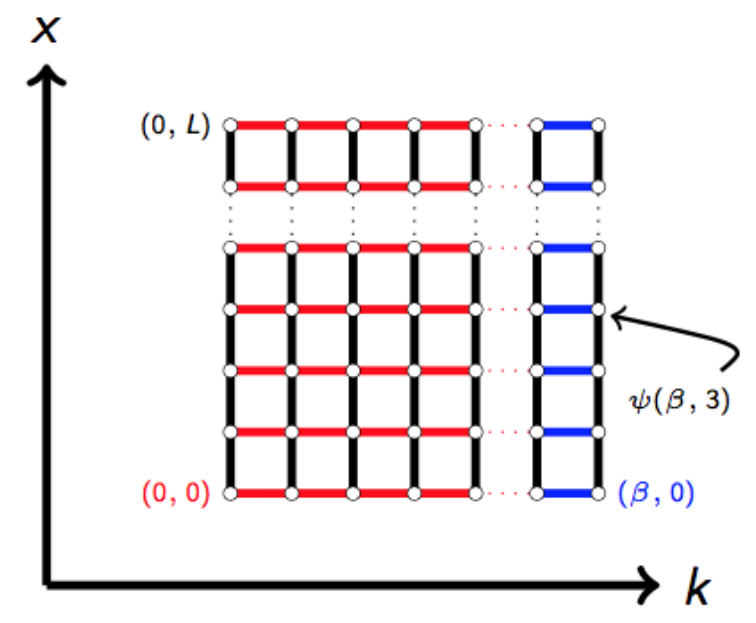

Figure 5.1: Gauge field configuration 
Let $(x, k)$ label a site on a $L \times \beta$ lattice with $x$ being the space location and $k$ being the Euclidean time location. The space link variables will be labeled by $U(x, k)$ and the time link variables connecting $(x, \beta)$ and $(x, 1)$ will be labeled by $V(x)$.

The standard Wilson plaquette gauge action with lattice gauge coupling, $b$, can be written as

$$
\begin{aligned}
& S_{g}=b N \sum_{x=1}^{L} \sum_{k=1}^{n}\left(S_{g}(x, k)\right) \\
& S_{g}(x, k)=\operatorname{Tr}\left[U(x, k) U^{\dagger}(x, k+1)+U(x, k+1) U^{\dagger}(x, k)\right] ; k \in[1, \beta-1] \\
& S_{g}(x, n)=\operatorname{Tr}\left[U(x, \beta) V(x+1)\{V(x) U(x, 1)\}^{\dagger}+V(x) U(x, 1)\{U(x, \beta) V(x+1)\}^{\dagger}\right]
\end{aligned}
$$

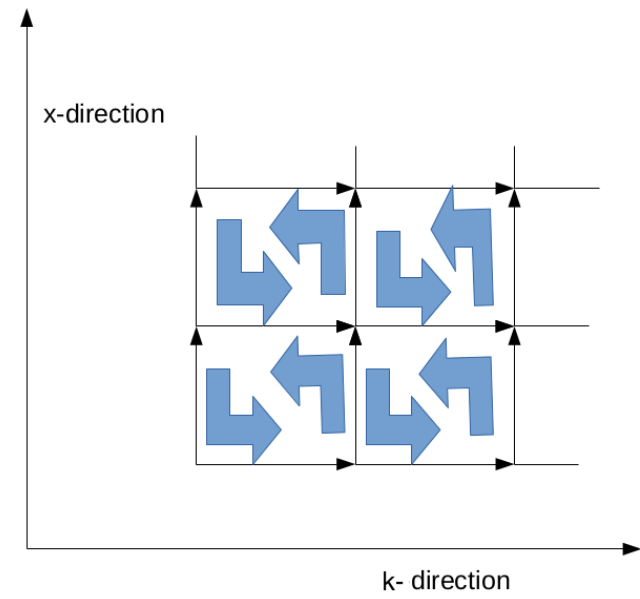

Figure 5.2: Fundamental plaquettes

\subsection{Fermions on the lattice}

In this section, the fermion doubling problem on the lattice will be discussed in general and the solution because of Wilson will be presented. The solution will be used in the later section to obtain the canonical formalism of QCD on a two dimensional lattice. 
The action for free fermion in an even dimensional Euclidean space-time is given by

$$
S_{F}^{0}[\psi, \bar{\psi}]=\int d^{d} x \bar{\psi}(x)\left(\gamma_{\mu} \partial_{\mu}+m\right) \psi(x)
$$

We use the Dirac basis for d-dimensions:

$$
\gamma_{j}=\left(\begin{array}{cc}
0 & \sigma_{j} \\
\sigma_{j} & 0
\end{array}\right) ; \gamma_{d}=\left(\begin{array}{cc}
0 & i \\
-i & 0
\end{array}\right) ; \gamma_{d+1}=\left(\begin{array}{cc}
1 & 0 \\
0 & 1
\end{array}\right)
$$

Where $j=1, \ldots ., d-1$ with $\left\{\sigma_{j}, \sigma_{k}\right\}=2 \delta_{j k} ; \sigma_{j}^{\dagger}=\sigma_{j} . \sigma$ matrices have dimension, $n_{s}=2^{\frac{d}{2}-1}$. Spinors are placed at lattice points and fermionic degrees of freedom are $\psi(n), \bar{\psi}(n)$. The naive derivative is

$$
\partial_{\mu} \psi(\mathbf{n}) \rightarrow \frac{\psi(\mathbf{n}+\hat{\mu})-\psi(\mathbf{n}-\hat{\mu})}{2}=\frac{1}{2}\left[\delta\left(\mathbf{n}^{\prime}, \mathbf{n}+\hat{\mu}\right)-\delta\left(\mathbf{n}^{\prime}, \mathbf{n}-\hat{\mu}\right)\right] \psi\left(\mathbf{n}^{\prime}\right) .
$$

On this discritization for $d$-dimension, matrix elements of Dirac operator is

$$
\begin{aligned}
D\left(\mathbf{n} \mid \mathbf{n}^{\prime}\right)_{\alpha \beta} & =m \delta_{\alpha \beta} \delta_{\mathbf{n n}^{\prime}} \\
& +\sum_{\mu=1}^{d}\left(\gamma_{\mu}\right)_{\alpha \beta} \frac{\delta\left(\mathbf{n}+\hat{\mu}, \mathbf{n}^{\prime}\right)-\delta\left(\mathbf{n}-\hat{\mu}, \mathbf{n}^{\prime}\right)}{2}
\end{aligned}
$$

Where, $\alpha, \beta=1,2, \ldots N_{s}$ are Dirac indices. The fermion action is

$$
S_{F}^{0}[\psi, \bar{\psi}]=\sum_{\mathbf{n}^{\prime}, \mathbf{n}} \sum_{\alpha, \beta} \bar{\psi}(\mathbf{n})_{\alpha} D\left(\mathbf{n} \mid \mathbf{n}^{\prime}\right)_{\alpha \beta} \psi\left(\mathbf{n}^{\prime}\right)_{\beta}
$$

Interactions with gauge fields are introduced by converting the derivative to a covariant derivative by

$$
D\left(\mathbf{n} \mid \mathbf{n}^{\prime}\right)_{\alpha \beta}^{a b}=\sum_{\mu=1}^{d}\left(\gamma_{\mu}\right)_{\alpha \beta} \frac{U_{\mu}(\mathbf{n})_{a b} \delta_{\mathbf{n}+\hat{\mu}, \mathbf{n}^{\prime}}-U_{\mu}^{\dagger}\left(\mathbf{n}^{\prime}\right)_{a b} \delta_{\mathbf{n}-\hat{\mu}, \mathbf{n}^{\prime}}}{2 a}+m \delta_{\alpha \beta} \delta_{a b} \delta_{\mathbf{n n}^{\prime}}
$$

where $a, b=1,2, \ldots N_{c}$ is color indices. 
One can use the Fourier basis to diagonalize the free Dirac operator and the operator for a momentum $p_{\mu}$ is

$$
D(p)=m \mathbf{1}+i \sum_{\mu=1}^{d} \gamma_{\mu} \sin \left(p_{\mu}\right)
$$

Dirac operator for massless fermions has a zero at $\mathbf{p}=(0,0)$ in continuum limit. But it has additional zeros for either $p_{\mu}=0$ or $p_{\mu}=\frac{\pi}{a}$. Therefore, there are $2^{d}$ zeros and it will describe that many massless fermions. This problem is referred to as the doubling problem [30].

In order to remove doublers, Wilson proposed adding an irrelevant term of the form $-(1 / 2) \sum_{\mu} \partial_{\mu} \partial_{\mu}$ to the Dirac Operator. With this term, the Wilson Dirac Operator takes the form

$$
\begin{aligned}
D\left(\mathbf{n} \mid \mathbf{n}^{\prime}\right)_{\alpha \beta}^{a b} & =(m+d) \delta_{\alpha \beta} \delta_{a b} \delta_{\mathbf{n n}^{\prime}} \\
& -\frac{1}{2} \sum_{\mu=1}^{d}\left(\mathbf{1}-\gamma_{\mu}\right)_{\alpha \beta} U_{\mu}(\mathbf{n})_{a b} \delta_{\mathbf{n}+\hat{\mu}, \mathbf{n}^{\prime}} \\
& -\frac{1}{2} \sum_{\mu=1}^{d}\left(\mathbf{1}+\gamma_{\mu}\right)_{\alpha \beta} U_{\mu}^{\dagger}(\mathbf{n})_{a b} \delta_{\mathbf{n}+\hat{\mu}, \mathbf{n}^{\prime}}
\end{aligned}
$$

The free Wilson Dirac operator in momentum space becomes

$$
\tilde{D}(p)=m \mathbf{1}+\sum_{\mu=1}^{d} \gamma_{\mu} \sin \left(p_{\mu}\right)+\underbrace{\sum_{\mu=1}^{d}\left(1-\cos \left(p_{\mu}\right)\right)}_{\text {Wilson }} .
$$

The massless operator still has a zero for $p_{\mu}=0$. The Wilson term is zero at zero momentum but it is not zero if one or more of the $p_{\mu}=\pi$. Therefore, the term acts like a mass term for all other zeros of the naive Dirac operator. 


\subsubsection{Wilson Dirac Operator on the two dimensional periodic Lattice in Weyl gauge}

In this section we set up the explicit matrix structure of Wilson Dirac operator on the two

dimensional periodic lattice in Weyl gauge. From (5.9), the matrix elements of Wilson Dirac Operator can be written as:

$$
\begin{aligned}
D\left(x, k \mid x^{\prime}, k^{\prime}\right)_{\alpha \beta}^{a b} & =(m+d) \delta_{\alpha \beta} \delta_{a b} \delta_{x x^{\prime}} \delta_{k k^{\prime}} \\
& -\frac{1}{2}\left(1-\gamma_{1}\right)_{\alpha \beta}[U]_{a b}(x, k) \delta\left(x+1, x^{\prime}\right) \delta_{k k^{\prime}} \\
& -\frac{1}{2}\left(1+\gamma_{1}\right)_{\alpha \beta}\left[U^{\dagger}\right]_{a b}(x, k) \delta\left(x-1, x^{\prime}\right) \delta_{k k^{\prime}} \\
& -\frac{1}{2}\left(1-\gamma_{2}\right)_{\alpha \beta}[V]_{a b}(x, k) \delta(k, n) \delta\left(k^{\prime}, 1\right) \delta_{x x^{\prime}} \\
& -\frac{1}{2}\left(1+\gamma_{2}\right)_{\alpha \beta}\left[V^{\dagger}\right]_{a b}(x, k) \delta_{x x^{\prime}} \delta(k, 1) \delta\left(k^{\prime}, n\right)
\end{aligned}
$$

We define operators at a fixed time, $k$,

$$
\begin{aligned}
B_{k} & =d+M-\frac{1}{2}\left(T_{1}(k)+T_{1}^{\dagger}(k)\right) \\
C_{k} & =\frac{1}{2}\left(T_{1}(k)-T_{1}^{\dagger}(k)\right) ; \quad C_{k}^{\dagger}=-C_{k} . \\
\left(T_{1}(k) \psi\right)(x) & =U(x, k) \psi(x+1) \\
\left(T_{1}^{\dagger}(k) \psi\right)(x) & =U^{\dagger}(x-1, k) \psi(x-1) ; \quad T_{1}^{\dagger} T_{1}=\mathbf{1} ;
\end{aligned}
$$

Let us also define the unitary opetator, $T_{2}$, by

$$
\left(T_{2} \psi\right)(x)=V(x) \psi(x)
$$


The Wilson-Dirac operator can be written in the matrix form

$$
D_{f}=\left(\begin{array}{cccccccccc}
B_{1} & C_{1} & 0 & 0 & 0 & 0 & \cdots & \cdots & T_{2}^{\dagger} & 0 \\
C_{1} & B_{1} & 0 & -1 & 0 & 0 & \cdots & \cdots & 0 & 0 \\
-1 & 0 & B_{2} & C_{2} & 0 & 0 & \cdots & \cdots & 0 & 0 \\
0 & 0 & C_{2} & B_{2} & 0 & -1 & \cdots & \cdots & 0 & 0 \\
0 & 0 & -1 & 0 & B_{3} & C_{3} & \cdots & \cdots & 0 & 0 \\
0 & 0 & 0 & 0 & C_{3} & B_{3} & \cdots & \cdots & 0 & 0 \\
\vdots & \vdots & \vdots & \vdots & \vdots & \ddots & \ddots & \ddots & \vdots & \vdots \\
\vdots & \vdots & \vdots & \vdots & \vdots & \vdots & \ddots & \ddots & \ddots & \vdots \\
0 & 0 & 0 & 0 & 0 & 0 & \cdots & \cdots & B_{\beta} & C_{\beta} \\
0 & T_{2} & 0 & 0 & 0 & 0 & \cdots & \cdots & C_{\beta} & B_{\beta}
\end{array}\right)
$$

\subsection{Fermion determinant}

In order to obtain an expression for the determinant of $D_{f}$, we perform the following two column manipulations:

$$
D_{f}^{\prime}=\left(\begin{array}{cccccccccc}
C_{1} & B_{1} & 0 & 0 & 0 & 0 & \cdots & \cdots & 0 & -T_{2}^{\dagger} \\
B_{1} & C_{1} & -1 & 0 & 0 & 0 & \cdots & \cdots & 0 & 0 \\
0 & -1 & C_{2} & B_{2} & 0 & 0 & \cdots & \cdots & 0 & 0 \\
0 & 0 & B_{2} & C_{2} & -1 & 0 & \cdots & \cdots & 0 & 0 \\
0 & 0 & 0 & -1 & C_{3} & B_{3} & \cdots & \cdots & 0 & 0 \\
0 & 0 & 0 & 0 & B_{3} & C_{3} & \cdots & \cdots & 0 & 0 \\
\vdots & \vdots & \vdots & \vdots & \vdots & \ddots & \ddots & \ddots & \vdots & \vdots \\
\vdots & \vdots & \vdots & \vdots & \vdots & \vdots & \ddots & \ddots & \ddots & \vdots \\
0 & 0 & 0 & 0 & 0 & 0 & \cdots & \cdots & C_{\beta} & B_{\beta} \\
-T_{2} & 0 & 0 & 0 & 0 & 0 & \cdots & \cdots & B_{\beta} & C_{\beta}
\end{array}\right)
$$




$$
D_{f}^{\prime \prime}=\left(\begin{array}{cccccccccc}
B_{1} & 0 & 0 & 0 & 0 & \cdots & \cdots & 0 & -T_{2}^{\dagger} & C_{1} \\
C_{1} & -1 & 0 & 0 & 0 & \cdots & \cdots & 0 & 0 & B_{1} \\
-1 & C_{2} & B_{2} & 0 & 0 & \cdots & \cdots & 0 & 0 & 0 \\
0 & B_{2} & C_{2} & -1 & 0 & \cdots & \cdots & 0 & 0 & 0 \\
0 & 0 & -1 & C_{3} & B_{3} & \cdots & \cdots & 0 & 0 & 0 \\
0 & 0 & 0 & B_{3} & C_{3} & \cdots & \cdots & 0 & 0 & 0 \\
\vdots & \vdots & \vdots & \vdots & \ddots & \ddots & \ddots & \vdots & \vdots & \vdots \\
\vdots & \vdots & \vdots & \vdots & \vdots & \ddots & \ddots & \ddots & \vdots & \vdots \\
0 & 0 & 0 & 0 & 0 & \cdots & \cdots & C_{\beta} & B_{\beta} & 0 \\
0 & 0 & 0 & 0 & 0 & \cdots & \cdots & B_{\beta} & C_{\beta} & -T_{2}
\end{array}\right)
$$

Let us define

$$
\begin{aligned}
& \alpha_{k}=\left(\begin{array}{cc}
B_{k} & 0 \\
C_{k} & -1
\end{array}\right) ; k=1, \ldots \beta, \\
& \delta_{k}=\left(\begin{array}{cc}
-1 & C_{k} \\
0 & B_{k}
\end{array}\right) ; k=1, \ldots \beta, \\
& X=\left(\begin{array}{cc}
1 & 0 \\
0 & T_{2}
\end{array}\right) ; Y=\left(\begin{array}{cc}
T_{2}^{\dagger} & 0 \\
0 & 1
\end{array}\right) .
\end{aligned}
$$

Then

$$
D_{f}(\mu)=\left(\begin{array}{ccccccc}
\alpha_{1} & 0 & 0 & \ldots & \ldots & 0 & \delta_{1} Y \\
\delta_{2} & \alpha_{2} & 0 & \ldots & \ldots & 0 & 0 \\
0 & \delta_{3} & \alpha_{3} & \ldots & \ldots & 0 & 0 \\
\ldots & \ldots & \ldots & . . & \ldots & \ldots & \ldots \\
\ldots & \ldots & \ldots & \ldots & \ldots & \ldots & \ldots \\
0 & 0 & 0 & \ldots & \ldots & \alpha_{\beta-1} & 0 \\
0 & 0 & 0 & \ldots & \ldots & \delta_{\beta} & \alpha_{\beta} X
\end{array}\right)
$$


Using result from [31], we get

$$
\operatorname{Det} D=\left(\prod_{j=1}^{\beta} \operatorname{det} \alpha_{j}\right) \operatorname{det}\left(X-\prod_{k=n}^{1}\left(-\alpha_{k}^{-1} \delta_{k}\right) Y\right)
$$

To understand the structure of $-\alpha_{k}^{-1} \delta_{k}$, we plug the expression for them as:

$$
\begin{aligned}
-\alpha_{k}^{-1} \delta_{k} & =-\left(\begin{array}{cc}
B_{k} & 0 \\
C_{k} & -1
\end{array}\right)^{-1}\left(\begin{array}{cc}
-1 & C_{k} \\
0 & B_{k}
\end{array}\right) \\
& =\left(\begin{array}{c|c}
B_{k}^{-1} & -B_{k}^{-1} C_{k} \\
\hline C_{k} B_{k}^{-1} & -C_{k} B_{k}^{-1} C_{k}+B_{k}
\end{array}\right) \\
& =\mathcal{T}_{k} .
\end{aligned}
$$

where

$$
\mathcal{T}_{k}=\left(\begin{array}{c|c}
B_{k}^{-1} & -B_{k}^{-1} C_{k} \\
\hline C_{k} B_{k}^{-1} & -C_{k} B_{k}^{-1} C_{k}+B_{k}
\end{array}\right)
$$

The inverse of $\mathcal{T}_{k}$ is

$$
\mathcal{T}_{k}^{-1}=\left(\begin{array}{c|c}
B_{k}-C_{k} B_{k}^{-1} C_{k} & C_{k} B_{k}^{-1} \\
\hline-B_{k}^{-1} C_{k} & B_{k}^{-1}
\end{array}\right)=\left(\begin{array}{cc}
0 & \mathbf{1} \\
\mathbf{1} & 0
\end{array}\right) \mathcal{T}_{k}\left(\begin{array}{cc}
0 & \mathbf{1} \\
\mathbf{1} & 0
\end{array}\right)=\Lambda \mathcal{T}_{k} \Lambda,
$$

and

$$
\Lambda=\Lambda^{\dagger} ; \quad \Lambda^{2}=1 ; \quad \operatorname{det} \mathcal{T}_{k}=1 ; \quad \mathcal{T}_{k}^{\dagger}=\mathcal{T}_{k}
$$

Using, $\operatorname{det} \alpha_{k}=\operatorname{det}\left(-B_{k}\right)$, one can arrive to the expression of fermion determinant,

$$
\operatorname{det} \mathbf{D}=\left(\prod_{j=1}^{\beta} \operatorname{det}\left(-B_{j}\right)\right) \times \operatorname{det}\left[T_{2} \mathbf{1}-\left(\prod_{k=n}^{1} \mathcal{T}_{k}\right)\right]
$$


Again using properties of $\Lambda$ and $\mathcal{T}_{k}$, one can get

$$
\operatorname{det} \mathbf{D}=\left(\prod_{j=1}^{\beta} \operatorname{det}\left(-B_{j}\right)\right) \operatorname{det}\left[\mathbf{1}-T_{2}\left(\prod_{k=1}^{n} \mathcal{T}_{k}\right)\right]
$$

By comparing (5.24) and (5.25), one can conclude that $\operatorname{det} \mathbf{D}_{f}$ is real and positive.

\subsubsection{Properties of $\mathcal{T}$}

We define a matrix $\mathcal{T}$ by

$$
\mathcal{T}=\left(\prod_{k=1}^{n} \mathcal{T}_{k}\right) \mathbf{1} T_{2}
$$

Where $\mathcal{T}_{k}$ and $T_{2}$ are such that $\operatorname{det}\left(T_{2}\right)=1, \operatorname{det}\left(\mathcal{T}_{k}\right)=1, \mathcal{T}_{k}^{\dagger}=\mathcal{T}_{k}$ and $\mathcal{T}_{k}^{-1}=\Lambda \mathcal{T}_{k} \Lambda$. Where $\Lambda^{\dagger}=\Lambda, \Lambda^{2}=\mathbf{1}$. Using these properties, it follows that $\operatorname{det}(\mathcal{T})=1$ and $\mathcal{T}$ is nither hermitian nor unitary and

$$
\mathcal{T}^{\dagger}=\Lambda \mathcal{T}^{-1} \Lambda
$$

Since $\mathcal{T}$ is a finite square matrix, all its right eigenvalues are the same as its left eigenvalues.

Let $\mathcal{R}$ be the matrix formed by all the right eigenvectors and let $\mathcal{D}$ be the diagonal matrix of all the eigenvalues. Then,

$$
\mathcal{T R}=\mathcal{R} \mathcal{D}
$$

Using above properties

$$
\mathcal{R}^{\dagger} \mathcal{T}^{\dagger}=\mathcal{D}^{\dagger} \mathcal{R}^{\dagger} \Longrightarrow \mathcal{D}^{-1 \dagger}\left(\mathcal{R}^{\dagger} \Lambda\right)=\left(\mathcal{R}^{\dagger} \Lambda\right) \mathcal{T}
$$

Thus it appears that, $\mathcal{R}^{\dagger} \Lambda$ is the matrix formed by all the left eigenvectors of $\mathcal{T}$. Since the set of right and left eigenvalues are the same, it follows that for every eigenvalue $\mathcal{D}_{i}$,

we have another eigenvalue $\frac{1}{\mathcal{D}_{i}^{*}}$. Assuming that no eigenvalue has a magnitude of unity and 
$\left|\mathcal{D}_{i}\right|>1$, let us assume that the columns of $\mathcal{R}$ is such that

$$
\mathcal{D}=\left(\begin{array}{cc}
\mathcal{C} & 0 \\
0 & \frac{1}{\mathcal{C}^{\dagger}}
\end{array}\right)
$$

Since $\mathcal{T} \mathcal{R}=\mathcal{R} \mathcal{D}$ and $\operatorname{det} \mathcal{T}=1$, it follows that

$$
1=\operatorname{det} \mathcal{D}=\frac{\operatorname{det} \mathcal{C}}{\operatorname{det} \mathcal{C}^{\dagger}} \Rightarrow \operatorname{det} \mathcal{C}=\operatorname{det} \mathcal{C}^{\dagger}
$$

Also

$$
\mathcal{D}^{-1 \dagger}=\left(\begin{array}{cc}
\frac{1}{\mathcal{C}^{\dagger}} & 0 \\
0 & \mathcal{C}
\end{array}\right)=\Lambda \mathcal{D} \Lambda
$$

From (5.29), we get

$$
\mathcal{D}^{-1 \dagger}\left(\mathcal{R}^{\dagger} \Lambda\right)=\left(\mathcal{R}^{\dagger} \Lambda\right) \mathcal{T} \Longrightarrow \mathcal{D}\left(\Lambda \mathcal{R}^{\dagger} \Lambda\right)=\left(\Lambda \mathcal{R}^{\dagger} \Lambda\right) \mathcal{T}
$$

Hence from (5.33), it is evident that, given the set of right eigenvectors $\mathcal{R},(\Lambda \mathcal{R} \Lambda)$ is a set of left eigenvectors that results in the left eigenvalues in the same order as the left. It also follows that $\Lambda \mathcal{R}^{\dagger} \Lambda \mathcal{R}$ is a diagonal matrix.

\subsubsection{Partition function}

In this section we want to construct the partition function using determinant obtained in (5.24). The property of $\mathcal{T}$ studied in previous topics help us to explore symmetry property under Euclidean time reversal and center transformation. Consider $N_{f}$ flavor theory with degenerate fermions. The partition function for $N_{f}$ flavour of fermions can be expressed as

$$
\begin{aligned}
Z= & \int d U(x, k) d V(x) e^{S_{g}}\left(\operatorname{det} D_{f}\right)^{N_{f}} \\
& =\int d U(x, k) d V(x) e^{S_{g}}\left(\prod_{j=1}^{n} \operatorname{det}\left(-B_{j}\right)\right)^{N_{f}} \operatorname{det}[\mathbf{1}-\mathcal{T}]^{N_{f}}
\end{aligned}
$$


Taking the term

$$
\operatorname{det}[\mathbf{1}-\mathcal{T}]^{N_{f}}=\operatorname{det}[\mathbf{1}-\mathcal{C}]^{N_{f}} \operatorname{det}\left[\mathbf{1}-\frac{1}{\mathcal{C}^{\dagger}}\right]^{N f}
$$

into expression of partition function, we get

$$
Z=\int d U(x, k) d V(x) e^{S_{g}}\left(\prod_{j=1}^{n} \operatorname{det}\left(-B_{j}\right)\right)^{N_{f}} \operatorname{det}[\mathbf{1}-\mathcal{C}]^{N f} \operatorname{det}\left[\mathbf{1}-\frac{1}{\mathcal{C}^{\dagger}}\right]^{N f}
$$

Taking this partition function we go through following steps to investigate symmetry property of partition function.

\section{Partition function under Euclidean time reversal}

Consider two gauge field configurations that are related by Euclidean time reversal: $U(x, k) \rightarrow$

$U(x, n-k+1)$ and $V(x) \rightarrow V^{\dagger}(x)$. Under this transformation, we want to observe how different quantities in (5.36) are affected. These are summarized in the following steps:

- Gauge action $S_{g}$ remains invariant.

- $\prod_{j=1}^{n} \operatorname{det}\left(-B_{j}\right)$ remains invariant.

- $\operatorname{det}[1-\mathcal{T}]$ becomes $\operatorname{det}\left[1-\mathcal{T}^{\dagger}\right]$.

The first two are easy to see. To see that the last one is true, note that

$$
\begin{aligned}
\mathcal{T}= & \left(\prod_{k=1}^{n} \mathcal{T}_{k}\right) \mathcal{T}_{d} \Rightarrow\left(\prod_{k^{\prime}=n}^{1} \mathcal{T}_{k}\right) \mathcal{T}_{d}^{\dagger}=\mathcal{T}_{d}\left[\mathcal{T}_{d}^{\dagger}\left(\prod_{k^{\prime}=n}^{1} \mathcal{T}_{k}\right)\right] \mathcal{T}_{d}^{\dagger} \\
& \therefore \mathcal{T} \rightarrow \mathcal{T}_{d} \mathcal{T}^{\dagger} \mathcal{T}_{d}^{\dagger}
\end{aligned}
$$


Therefore all eigenvalues of $\mathcal{T}$ goes to its complex conjugate and it follows that

$$
\operatorname{det}[\mathbf{1}-\mathcal{T}] \Rightarrow \operatorname{det}\left[\mathbf{1}-\mathcal{T}^{\dagger}\right]
$$

Above three facts helps us to see that the partition function is real. Let the eigenvalues of $\mathcal{R}$, namely, $\mathcal{D}_{i}$ be arranged such that $\left|\mathcal{D}_{i+1}\right|>\left|\mathcal{D}_{i}\right|$. We can write

$$
\begin{aligned}
& \operatorname{det}[\mathbf{1} 1-\mathcal{C}]^{N_{f}} \operatorname{det}\left[\mathbf{1}-\frac{1}{\mathcal{C}^{\dagger}}\right]^{N_{f}} \\
& =\left(\prod_{i=1}^{K}\left(1-\mathcal{D}_{i}\right)\right)^{N_{f}}\left(\prod_{i=j}^{K}\left(1-\frac{1}{D_{j}^{*}}\right)\right)^{N_{f}} \\
& =\left(\prod_{i=1}^{K} \sum_{s_{i}, r_{i}=0}^{N_{f}}\right) \prod_{i=1}^{K}\left(C_{s_{i}}^{N_{f}} C_{r_{i}}^{N_{f}} \frac{\left(-\mathcal{D}_{i}\right)^{N_{f}-s_{i}}}{\left(-\mathcal{D}_{i}^{*}\right)^{r_{i}}}\right) .
\end{aligned}
$$

We can write the partition function (5.36) as

$$
Z=\left(\prod_{i=1}^{K} \sum_{s_{i}, r_{i}=0}^{N_{f}}\right) Z_{\left\{s_{i} r_{i}\right\}}
$$

where $\left\{s_{i}, r_{i}\right\}$ represent different sets $\left(s_{1}, \ldots s_{K} ; r_{1}, \ldots r_{K}\right)$ of all possible combinations made by picking number of flavors from $0,1,2 \ldots N_{f}$. Each

$$
\begin{aligned}
& Z_{\left\{s_{i} r_{i}\right\}}=\int d U_{i}(x, k) d U_{d}(x) e^{S_{g}}\left(\prod_{j=1}^{n} \operatorname{det}\left(-B_{j}\right)\right)^{N_{f}} \\
& \times(\operatorname{det}(-\mathcal{C}))^{N_{f}} \prod_{i=1}^{K}\left(C_{s_{i}}^{N_{f}} C_{r_{i}}^{N_{f}} \frac{1}{\left(-\mathcal{D}_{i}\right)^{s_{i}}\left(-\mathcal{D}_{i}^{*}\right)^{r_{i}}}\right) .
\end{aligned}
$$

\section{Partition function under global $Z_{N}$ transformation}

Consider gauge fields related by a center transformation:

$$
V(x) \rightarrow e^{i \frac{2 \pi k}{N}} V(x) ; k=0, \ldots, N-1 .
$$

Under this transformation: 
- Gauge action $S_{g}$ remains invariant

- $\operatorname{det}\left(-B_{j}\right)$ remains invariant

- All eigen values of $\mathcal{T}$ are multiplied by phase $e^{i \frac{2 \pi k}{N}}$.

The last statement follows from the following reasoning:

$$
T_{d}(x) \rightarrow e^{i \frac{2 \pi k}{N}} T_{d}(x)
$$

Further we have defined matrix $\mathcal{T}$ as

$$
\mathcal{T}=\left(\prod_{k=1}^{n} \mathcal{T}_{k}\right) \mathbf{1} T_{d}
$$

Which implies under this transformation all eigen values of $\mathcal{T}$ are multiplied by phase $e^{i \frac{2 \pi k}{N}}$ i.e.,

$$
\mathcal{D}_{i} \rightarrow e^{i \frac{2 \pi k}{N}} \mathcal{D}_{i}
$$

This implies

$$
\operatorname{det}(-\mathcal{C}) \rightarrow \operatorname{det}(-\mathcal{C})
$$

With all these contributions $Z_{\left\{s_{i} r_{i}\right\}}$ transforms as

$$
Z_{\left\{s_{i} r_{i}\right\}} \rightarrow e^{i \frac{2 \pi k}{N}\left[\sum_{i}^{K}\left(r_{i}-s_{i}\right)\right]} Z_{\left\{s_{i} r_{i}\right\}}
$$

Averaging over all $k$ will result in a zero unless $\sum_{i=1}^{K}\left(r_{i}-s_{i}\right)$ is a multiple of $N$.

\section{A double expansion}

Setting

$$
\sum_{i=1}^{K}\left(r_{i}-s_{i}\right)=N Q
$$




$$
\sum_{i=1}^{K}\left(s_{i}+r_{i}\right)=P
$$

we see that $P$ is the quark number (number of quarks and anti-quarks) and $Q$ is the baryon number. Where range of $P$ goes from 0 to $2 K N_{f}$. We can write

$$
Z=\sum_{P, Q} Z_{P Q}
$$

with

$$
Z_{P Q}=\left(\prod_{i=1}^{K} \sum_{s_{i}, r_{i}=0}^{N_{f}}\right) \delta\left(\sum_{i=1}^{K}\left(r_{i}-s_{i}\right)-N Q\right) \delta\left(\sum_{i=1}^{K}\left(r_{i}+s_{i}\right)-P\right) Z_{\left\{s_{i} r_{i}\right\}}
$$

\subsubsection{Special cases: $Z_{00}, Z_{20}, Z_{31}$}

In this section we will calculate the ratios of $Z_{20}, Z_{31}$ over $Z_{00}$ which appear as expectation value of some operator product. This is the benefit that we obtain from special structure of the partition function (5.50) and (5.51).

\section{Calculation of $Z_{00}$}

For $P=0$ and $Q=0$, using (5.48) and (5.49), the possible values of $s_{i}$ and $r_{i}$ are 0 . Which means the collection of configurations $\left\{s_{i}, r_{i}\right\}$ which includes the configurations satisfying above equations is only one configuration $\left\{s_{1}=0, s_{2}=0 \ldots s_{K}=0 ; r_{1}=0, r_{2}=0 \ldots, r_{K}=0\right\}$

$$
\left\{s_{i}, r_{i}\right\}=\{(0,0,0 \ldots 0 ; 0,0,0, \ldots, 0)\} .
$$

Therefore we end up with

$$
\begin{aligned}
Z_{00}=Z_{\left\{s_{i} r_{i}\right\}} & =\int d U_{i}(x, k) d U_{d}(x) e^{S_{g}}\left(\prod_{j=1}^{n} \operatorname{det}\left(-B_{j}\right)\right)^{N_{f}} \\
\times & (\operatorname{det}(-\mathcal{C}))^{N_{f}} \prod_{i=1}^{K}\left(C_{0}^{N_{f}} C_{0}^{N_{f}} \frac{1}{\left(-\mathcal{D}_{i}\right)^{0}\left(-\mathcal{D}_{i}^{*}\right)^{0}}\right)
\end{aligned}
$$


For $N_{f}=2$ :

$$
Z_{00}=\int d U_{i}(x, k) d U_{d}(x) e^{S_{g}}\left(\prod_{j=1}^{n} \operatorname{det}\left(-B_{j}\right)\right)^{2}(\operatorname{det}(-\mathcal{C}))^{2}
$$

\section{Calculation of $Z_{20}$}

For $P=2$ and $Q=0$, using (5.48) and (5.49), the possible values of $s_{i}$ and $r_{i}$ are 1. Which means the collection of configurations $\left\{s_{i}, r_{i}\right\}$ which includes the configurations satisfying above equations are

\begin{tabular}{|c|}
\hline$s_{1}=1, r_{1}=1$ rest of all $s_{i}, r_{i}=0$ \\
\hline$s_{1}=1, r_{2}=1$ rest of all $s_{i}, r_{i}=0$ \\
\hline$\ldots \ldots \ldots$ \\
\hline$s_{1}=1, r_{K}=1$ rest of all $s_{i}, r_{i}=0$ \\
\hline$\ldots$ and so on $\ldots$ \\
\hline$s_{K}=1, r_{K}=1$ rest of all $s_{i}, r_{i}=0$ \\
\hline
\end{tabular}

Thus in total there are $K^{2}$ configurations in $\left\{s_{i}, r_{i}\right\}$. Let us consider the first configuration: $s_{1}=1, r_{1}=1$. With $N_{f}=2$, the specific form of $Z_{\left\{s_{i} r_{i}\right\}}$ is

$$
\begin{aligned}
Z_{\left\{s_{i} r_{i}\right\}} & =\int d U_{i}(x, k) d U_{d}(x) e^{S_{g}}\left(\prod_{j=1}^{n} \operatorname{det}\left(-B_{j}\right)\right)^{2} \\
& \times(\operatorname{det}(-\mathcal{C}))^{2} \prod_{i=1}^{K}\left(C_{s_{i}}^{2} C_{r_{i}}^{2} \frac{1}{\left(-\mathcal{D}_{i}\right)^{s_{i}}\left(-\mathcal{D}_{i}^{*}\right)^{r_{i}}}\right)
\end{aligned}
$$

Consider the relevant term, $\prod_{i=1}^{K}\left(C_{s_{i}}^{2} C_{r_{i}}^{2} \frac{1}{\left(-\mathcal{D}_{i}\right)^{s_{i}\left(-\mathcal{D}_{i}^{*}\right)^{r_{i}}}}\right)$. Expanding it to $\mathrm{K}$ terms and by plugging all $s_{i}$ and $r_{i}$ one can get

$$
\prod_{i=1}^{K}\left(C_{s_{i}}^{2} C_{r_{i}}^{2} \frac{1}{\left(-\mathcal{D}_{i}\right)^{s_{i}}\left(-\mathcal{D}_{i}^{*}\right)^{r_{i}}}\right)=4\left(\frac{1}{\mathcal{D}_{1} \mathcal{D}_{1}^{*}}\right)
$$


Similarly considering other relevant terms one can obtain the total contribution denoted as $\zeta_{20}$.

$$
\zeta_{20}=4\left[\left(\frac{1}{\mathcal{D}_{1} \mathcal{D}_{1}^{*}}+\ldots+\frac{1}{\mathcal{D}_{1} \mathcal{D}_{K}^{*}}\right)+\ldots+\left(\frac{1}{\mathcal{D}_{K} \mathcal{D}_{1}^{*}}+\ldots+\frac{1}{\mathcal{D}_{K} \mathcal{D}_{K}^{*}}\right)\right]
$$

i.e.

$$
\zeta_{20}=4 \sum_{i j}^{K} \frac{1}{D_{i} D_{j}^{*}}
$$

Finally, with this result $Z_{20}$ can be expressed as

$$
Z_{20}=\int d U_{i}(x, k) d U_{d}(x) e^{S_{g}}\left(\prod_{j=1}^{n} \operatorname{det}\left(-B_{j}\right)\right)^{2}(\operatorname{det}(-\mathcal{C}))^{2}\left(4 \sum_{i j}^{K} \frac{1}{D_{i} D_{j}^{*}}\right)
$$

Using (5.53) and (5.58), the ratio $\frac{Z_{20}}{Z_{00}}$ becomes

$$
\frac{Z_{20}}{Z_{00}}=\frac{\int d U_{i}(x, k) d U_{d}(x) e^{S_{g}}\left(\prod_{j=1}^{n} \operatorname{det}\left(-B_{j}\right)\right)^{2}(\operatorname{det}(-\mathcal{C}))^{2}\left(4 \sum_{i j}^{K} \frac{1}{D_{i} D_{j}^{*}}\right)}{\int d U_{i}(x, k) d U_{d}(x) e^{S_{g}}\left(\prod_{j=1}^{n} \operatorname{det}\left(-B_{j}\right)\right)^{2}(\operatorname{det}(-\mathcal{C}))^{2}}
$$

Obviously,

$$
\frac{Z_{20}}{Z_{00}}=\left\langle\left(4 \sum_{i j}^{K} \frac{1}{D_{i} D_{j}^{*}}\right)\right\rangle
$$

\section{Calculation of $Z_{31}$}

For $P=3$ and $Q=1$, using (5.48) and (5.49), the possible values of $s_{i}$ and $r_{i}$ are: 0 for all $s_{i}$ and $(1,1,1)$ or $(1,2)$ for $r_{i}$. Which means the collection of configurations $\left\{s_{i}, r_{i}\right\}$ which includes the configurations satisfying above equations are of two types:

- Type -I: $r_{i}=(1,1,1)$, all $s_{i}=0$ 
There are total of $\frac{K(K-1)(K-2)}{3 !}$ configurations in $\left\{s_{i}, r_{i}\right\}$ of this type. For example consider the configuration: $r_{1}=1, r_{2}=1, r_{3}=1$ rest of all $s_{i}$ and $r_{i}=0$. Consider the relevant term: $\prod_{i=1}^{K}\left(C_{s_{i}}^{2} C_{r_{i}}^{2} \frac{1}{\left(-\mathcal{D}_{i}\right)^{s_{i}\left(-\mathcal{D}_{i}^{*}\right)^{r_{i}}}}\right)$. On expanding it to $\mathrm{K}$ terms and plugging values of all $s_{i}$ and $r_{i}$, one can get

$$
\prod_{i=1}^{K}\left(C_{s_{i}}^{2} C_{r_{i}}^{2} \frac{1}{\left(-\mathcal{D}_{i}\right)^{s_{i}}\left(-\mathcal{D}_{i}^{*}\right)^{r_{i}}}\right)=-8 \frac{1}{\left(\mathcal{D}_{1}^{*} \mathcal{D}_{2}^{*} \mathcal{D}_{3}^{*}\right)}
$$

Considering all relevant term the total Contribution denoted by $\zeta_{31}^{[111]}$ becomes

$$
\zeta_{31}^{[111]}=-8\left[\frac{1}{\left(\mathcal{D}_{1}^{*} \mathcal{D}_{2}^{*} \mathcal{D}_{3}^{*}\right)}+\ldots+\frac{1}{\left(\mathcal{D}_{K-2}^{*} \mathcal{D}_{K-1}^{*} \mathcal{D}_{K}^{*}\right)}\right]
$$

i.e.

$$
\zeta_{31}^{[111]}=-\frac{8}{3 !} \sum_{i \neq j \neq k}^{K} \frac{1}{D_{i}^{*} D_{j}^{*} D_{k}^{*}}=-\frac{8}{3 !}\left(\sum_{i j k}^{K} \frac{1}{D_{i}^{*} D_{j}^{*} D_{k}^{*}}-3 \sum_{i j}^{K} \frac{1}{D_{i}^{* 2} D_{j}^{*}}+2 \sum_{k}^{K} \frac{1}{D_{k}^{* 3}}\right)
$$

- Type -II: $r_{i}=(1,2)$, all $s_{i}=0$

There are total of $K(K-1)$ configurations of this type in $\left\{s_{i}, r_{i}\right\}$. For example consider the configuration: $r_{1}=1, r_{2}=2$ rest of all $s_{i}$ and $r_{i}=0$. Consider the relevant term: $\prod_{i=1}^{K}\left(C_{s_{i}}^{2} C_{r_{i}}^{2} \frac{1}{\left(-\mathcal{D}_{i}\right)^{s_{i}\left(-\mathcal{D}_{i}^{*}\right)^{r_{i}}}}\right)$. Expanding it to $\mathrm{K}$ terms and plugging for $s_{i}$ and $r_{i}$, one can get

$$
\prod_{i=1}^{K}\left(C_{s_{i}}^{2} C_{r_{i}}^{2} \frac{1}{\left(-\mathcal{D}_{i}\right)^{s_{i}}\left(-\mathcal{D}_{i}^{*}\right)^{r_{i}}}\right)=-2 \frac{1}{\mathcal{D}_{1}^{*}\left(\mathcal{D}_{2}^{*}\right)^{2}}
$$

Considering all relevant term the total Contribution denoted by $\zeta_{31}^{[12]}$ becomes

$$
\zeta_{31}^{[12]}=-2\left[\frac{1}{\mathcal{D}_{1}^{*}\left(\mathcal{D}_{2}^{*}\right)^{2}}+\ldots+\frac{1}{\mathcal{D}_{K}^{* 2} \mathcal{D}_{K-1}^{*}}\right]
$$


i.e.

$$
\zeta_{31}^{[12]}=-2 \sum_{i \neq j}\left(\frac{1}{D_{i}^{*} D_{j}^{* 2}}\right)=-2\left[\sum_{i j}\left(\frac{1}{D_{i}^{*} D_{j}^{* 2}}\right)-\sum_{i} \frac{1}{D_{i}^{* 3}}\right]
$$

Thus total contribution from both type I and II gives us :

$$
Z_{31}=\int d U_{i}(x, k) d U_{d}(x) e^{S_{g}}\left(\prod_{j=1}^{n} \operatorname{det}\left(-B_{j}\right)\right)^{2}(\operatorname{det}(-\mathcal{C}))^{2}\left(\zeta_{31}^{[111]}+\zeta_{31}^{[12]}\right)
$$

i.e.

$$
\frac{Z_{31}}{Z_{00}}=\left\langle\left(\zeta_{31}^{[111]}+\zeta_{31}^{[12]}\right)\right\rangle
$$

Using (5.63) and (5.66) for total contribution, we can reexpress this ratio as

$$
\frac{Z_{31}}{Z_{00}}=\left\langle-\frac{4}{3}\left(\sum_{i}^{K} \frac{1}{D_{i}^{*}}\right)^{3}+2\left(\sum_{i}^{K} \frac{1}{D_{i}^{* 2}}\right)\left(\sum_{j}^{K} \frac{1}{D_{j}^{*}}\right)-\frac{2}{3} \sum_{k}^{K} \frac{1}{D_{k}^{* 3}}\right\rangle
$$

\subsection{Algorithms for numerical calculation}

Let $\Pi_{1}(\mathbf{x}, k)$ be traceless Hermitian matrices that correspond to momentum conjugate to $U(x, k)$ and let $\Pi_{2}(\mathbf{x})$ be a traceless Hermitian matrices that correspond to momentum conjugate to $V(x)$. We want to use Hybrid Monte Carlo (HMC) method [32] to generate gauge fields for which we need to find canonical equation of motion. We start with constructing new action by taking kinetic energy part of the action as

$$
S_{k}=-\frac{1}{2} \sum_{x, k} \operatorname{Tr} \Pi_{1}^{2}(\mathbf{x}, k)-\frac{1}{2} \sum_{\mathbf{x}} \operatorname{Tr} \Pi_{2}^{2}(\mathbf{x}) .
$$

Potential energy part of the action as

$$
S_{p}=S_{g}+N_{f} \ln \operatorname{det} H_{f}
$$

Where $S_{g}$ is gauge action from (5.1) and $H_{f}=\gamma_{3} D_{f}$ is a Hermitian Dirac operator . 


\subsubsection{Canonical equations of motion for HMC}

The equation of motion for variables $U_{i}(\mathbf{x}, k)$ and $V(\mathbf{x})$ are:

$$
\begin{aligned}
& \frac{d U(\mathbf{x}, k)}{d \tau}=i \Pi_{1}(\mathbf{x}, k) U(\mathbf{x}, k) \\
& \frac{d V(\mathbf{x})}{d \tau}=i \Pi_{2}(\mathbf{x}) V(\mathbf{x})
\end{aligned}
$$

Then, conservation of energy; $\frac{d\left(S_{k}+S_{p}\right)}{d \tau}=0$ implies

$$
\begin{aligned}
& \sum_{x, k} \operatorname{Tr}\left[\Pi_{1}(x, k) \frac{d \Pi_{1}(x, k)}{d \tau}\right]+\sum_{x} \operatorname{Tr}\left[\Pi_{2}(x) \frac{d \Pi_{2}(x)}{d \tau}\right] \\
= & \frac{d S_{g}}{d \tau}+N_{f} \operatorname{Tr}\left[H_{f}^{-1} \frac{d H_{f}}{d \tau}\right] \\
= & \sum_{x, k} \operatorname{Tr}\left[\Pi_{1}(x, k)\left\{b N F_{g}^{1}(x, k)+N_{f} F_{f}^{1}(x, k)\right\}\right] \\
+ & \sum_{x} \operatorname{Tr}\left[\Pi_{2}(x)\left\{b N F_{g}^{2}(x)+N_{f} F_{f}^{2}(x)\right\}\right]+\text { h.c. }
\end{aligned}
$$

Noting that the conjugate momenta are hermitian and traceless, it follows that

$$
\frac{d \Pi_{1}(x, k)}{d \tau}=b N F_{g}^{1}(x, k)+N_{f} F_{f}^{1}(x, k)
$$

and

$$
\frac{d \Pi_{2}(x)}{d \tau}=b N F_{g}^{2}(x)+N_{f} F_{f}^{2}(x)
$$

Using the Wilson gauge action

$$
\begin{aligned}
F_{g}^{1}(x, k)= & U_{i}(x, k) F_{g e}^{1}(x, k) \\
& -\frac{1}{N} \operatorname{Tr}\left[U_{i}(x, k) F_{g e}^{1}(x, k)\right]+\text { h.c. } \\
F_{g e}^{1}(x, k)= & i\left[U^{\dagger}(x, k+1)+U^{\dagger}(\mathbf{x}, k-1)\right] \quad k \in[2, \beta-1] \\
F_{g e}^{1}(x, 1)= & i\left[U^{\dagger}(x, 2)+V^{\dagger}(x+1) U^{\dagger}(x, \beta) V(x)\right] \\
F_{g e}^{1}(x, \beta)= & i\left[V(x+1) U^{\dagger}(x, 1) V^{\dagger}(x)+U^{\dagger}(x, \beta-1)\right] \\
F_{g}^{2}(x)= & U_{d}(x) F_{g e}^{2}(x)-\frac{1}{N} \operatorname{Tr}\left[V(x) F_{g e}^{2}(x)\right]+\text { h.c }
\end{aligned}
$$




$$
\begin{aligned}
F_{g e}^{2}(x)= & i\left[U(x, 1) V^{\dagger}(x+1) U^{\dagger}(x, \beta)\right. \\
& \left.+U^{\dagger}(x-1,1) V^{\dagger}(x-1) U(x-1, \beta)\right]
\end{aligned}
$$

Using (5.11)

$$
\begin{array}{r}
F_{f}^{1}(x, k)=U(x, k) \bar{F}_{f}^{1}(x, k)-\frac{1}{N} \operatorname{Tr}\left[U(x, k) \bar{F}_{f}^{1}(x, k)\right]+\text { h.c. } \\
\bar{F}_{f}^{1}(x, k)=-\frac{i}{2}\left(\operatorname{Tr}\left(\left(1-\gamma_{i}\right) \gamma_{3} H_{f}^{-1}\right)\right)(x+1, k ; x, k)
\end{array}
$$

and

$$
\begin{aligned}
F_{f}^{2}(x, k) & =V(x) \bar{F}_{f}^{2}(x)-\frac{1}{N} \operatorname{Tr}\left[V(x) \bar{F}_{f}^{2}(x)\right]+\text { h.c. } \\
\bar{F}_{f}^{2}(x) & =-\frac{i}{2}\left\{\operatorname{Tr}\left[\left(1-\gamma_{2}\right) \gamma_{3} H_{f}^{-1}\right]\right\}(x, \beta ; x, 1) .
\end{aligned}
$$

With these numerical details in place, we developed the numerical code to generate gauge field configurations for SU(3) gauge theory with two degenerate flavors. This enabled us to compute the physical spectrum of this theory. The results are presented in the next chapter. 


\section{CHAPTER 6}

\section{Observables and results from numerical calculations}

\subsection{Parameters}

The parameters that enter the numerical simulation described in section 4 of chapter 4 are the gauge group, $N$; number of fermion flavors, $N_{f}$; lattice gauge coupling, $b$; spatial extent of the lattice, $L$; temporal extent of the lattice, $\beta$; and the lattice fermion mass, $m$. All the results obtained are for the gauge group, $N=3$. Mesons are made up of a quark and anti-quark and baryons are made up of three quarks; mesons are bosons and baryons are fermions. We will restrict ourselves to two flavors of quarks, namely, $N_{f}=2$; and we will assume that they are degenerate (same quark masses). Chiral symmetry cannot be broken in two dimensions because of Elitzur's theorem $[9,30]$ and therefore we do not have to distinguish between the flavor content of the different mesons; they will all be degenerate. The same will be true of baryons. The quark mass can be negative or positive. We do not expect the sign to be relevant in two dimensions since there is no topological term to the gauge action in two dimensional QCD.

The continuum limit will be obtained by studying three different lattice couplings, $b=$ $0.25,0.375$ and 0.5 . These values of coupling constants were sufficient for us to demonstrate that our results have reached the continuum limit within statistical errors of the simulation. At the fixed lattice coupling, we studied three different spatial extents, $L=4,5$ and 6 , to demonstrate that our results have reached the values for infinite spatial extents. For each choice of $b$ and $L$, we obtained results at several different temporal extents, $\beta=$ $4,6,8,10,12,14,16$. Focussing on the special cases, $Z_{00}, Z_{20}$ and $Z_{31}$, described in section 4.4 of chapter 4 , we will extract the meson and baryon masses using the formulas,

$$
\frac{Z_{20}}{Z_{00}}=e^{-m_{M}\left(m_{q}\right) \frac{\beta}{L}} ; \quad \frac{Z_{31}}{Z_{00}}=e^{-m_{B}\left(m_{q}\right) \frac{\beta}{L}} .
$$

The lattice mass, $m$, is related to the dimensionless quark mass, $m_{q}$, by $m=\frac{m_{q}}{L}$. In what follows, we will show that even though $m_{M}\left(m_{q}\right)$ depends upon $\beta$ at a fixed, $b, m_{q}$ and $L$, it 
has approached the limit at infinite $\beta$. Our aim will then be to use the values at the largest $\beta$, namely; $\beta=16$, as the values for the meson and baryon masses at a fixed $\beta, L$ and $m_{q}$.

Since we have extracted a factor of $\frac{\beta}{L}$, in equation (1), the meson and baryons masses are dimensionless measured in units of the physical coupling.

We proceed to provide details of our results in a systematic manner in the following sections. All our HMC runs had the following parameters. The hundred steps of evolution of the equation of motion were used to thermalize the gauge field configuration where each step started with a new set of $\Pi_{1}(\mathbf{x}, k)$ and $\Pi_{2}(\mathbf{x})$ drawn from a normal distribution as defined in section 5.4 of chapter 5. Each step of classical evolution was obtained using 100 discrete steps such that the total evolution extent was unity. These computation steps were sufficient to obtain a conservation of the total energy within acceptable limits. A total number of 100 measurements were obtained after thermalization where gauge fields between two consecutive measurements corresponded to one step of classical evolution. All results shown in the next sections have error bars. These are obtained using the standard statistical estimate assuming all measurements are independent.

\subsection{Evidence for the $\beta \rightarrow \infty$ limit}

Given a gauge field configuration, a typical mesonic state has all possible mesons and not just the ground state mesons. The same is the case for baryons. Since the mesonic state and baryonic states are different in the quark content, $P=2, Q=0$ for mesons and $P=3, Q=1$ for baryons, baryons will not contribute to a meson measurement and vice-versa. In order to obtain the ground state in the meson and baryon sector, we need to evolve for a certain extent in $\beta$ for the ground state to dominate. The four plots, Figures 1-8, show samples of the data. Several points are evident from the plots:

1. The statistical errors in meson masses (Figures 6.1, 6.2,6.3 and 6.4) are consistently less that the ones in the baryon masses (Figures 6.5, 6.6, 6.7 and 6.8). The reason for this is evident from a comparison of equation (5.60) and equation (5.69). The result for $Z_{20}$ only involves a sum of positive numbers. The result for $Z_{31}$ is complex and is made real by adding up the contribution from a gauge field and its partner under 
Euclidean time reversal. As such, the statistical average over gauge configurations for mesons comes from a set of positive real numbers and from just a set of real numbers for baryons. Even though the statistical average for the baryons is positive, larger errors are expected and this is what is seen in Figure 6.5, 6.6, 6.7 and 6.8).

2. All figures show a dependence of the meson and fermion masses on $\beta$ but a plateau (almost an independence in $\beta$ within two standard deviations) is seen in all plots. Therefore, we are justified to use the value at $\beta=16$ as the mass at infinite $\beta$.

3. The plateau is obtained at an earlier value of $\beta$ for smaller values of quark masses suggesting a larger split in the excited to ground state.

4. The plateau is obtained sooner at larger values of $L$ suggesting that the ground state and excited states are well separated for larger spatial extents.

\subsection{Approach to the infinite spatial extent}

Figures $6.9,6.10$ and 6.11 show that behavior of the lightest pion mass as a function of the quark mass at $b=0.25,0.375,0.5$ respectively. The values for the pion mass are taken from the $\beta=16$ data. All three plots show a clear dependence of the pion mass on the quark mass since the error bars are small. In addition, the effect of finite spatial extent is small since all three data sets essentially fall on top of each other in figures $6.9,6.10$ and 6.11. We will use the $L=6$ data in the next section to study the approach to the continuum limit.

Figures $6.12,6.13$ and 6.14 show that behavior of the lightest baryon mass as a function of the quark mass at $b=0.25,0.375,0.5$ respectively. The values for the baryon mass are taken from the $\beta=16$ data. All three plots show large error bars as expected from the discussion in the previous section. In spite of this, one can see a trend in the behavior of the baryon mass as a function of the quark mass. The effect of finite spatial extent, of any, is difficult to see due to the large error bars. We will use the $L=6$ data in the next section to study the approach to the continuum limit. 


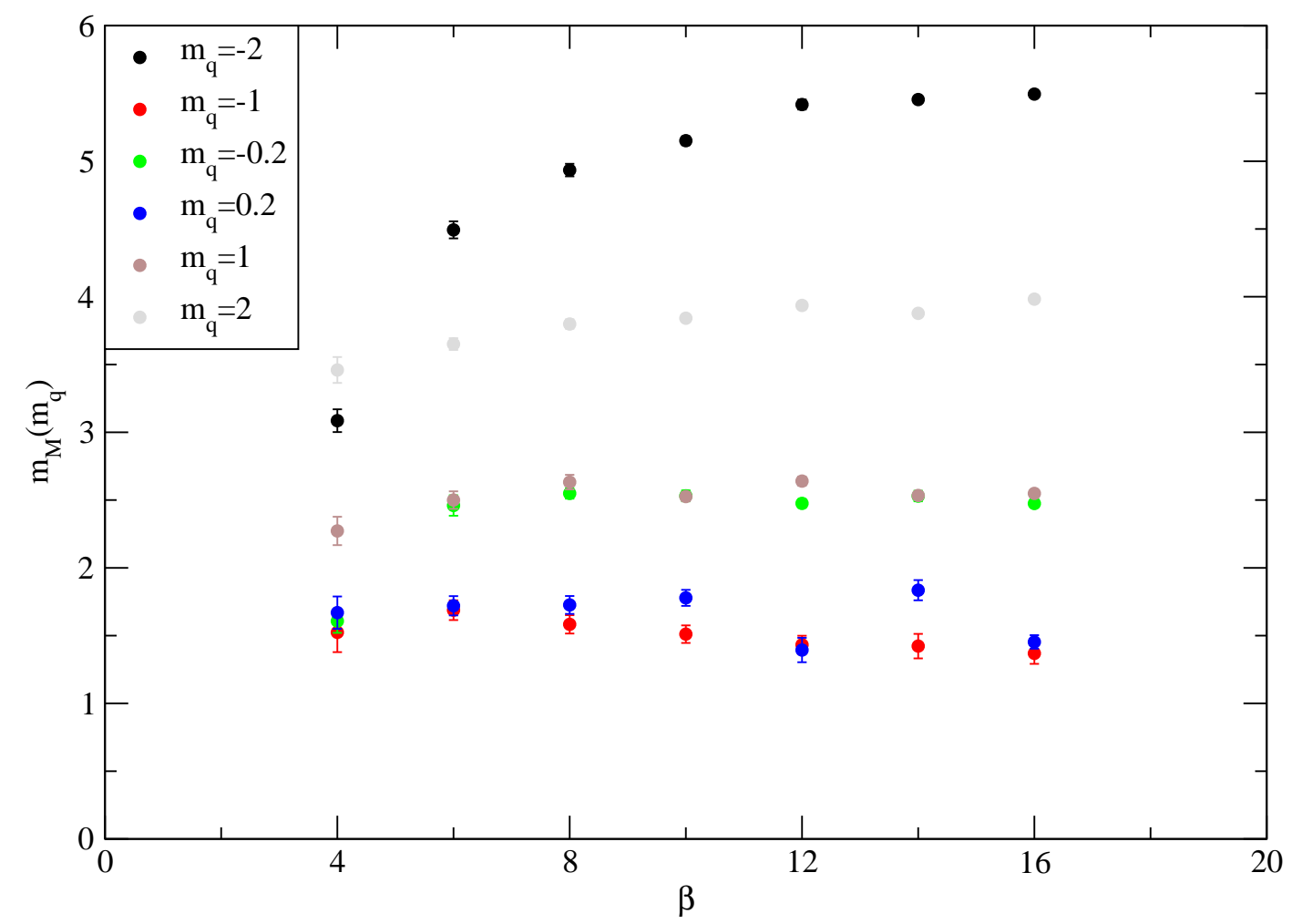

Figure 6.1: A plot of $m_{M}\left(m_{q}\right)$ as a function of $\beta$ at $L=4$ and $b=0.25$ at six different sample values of $m_{q}$. 


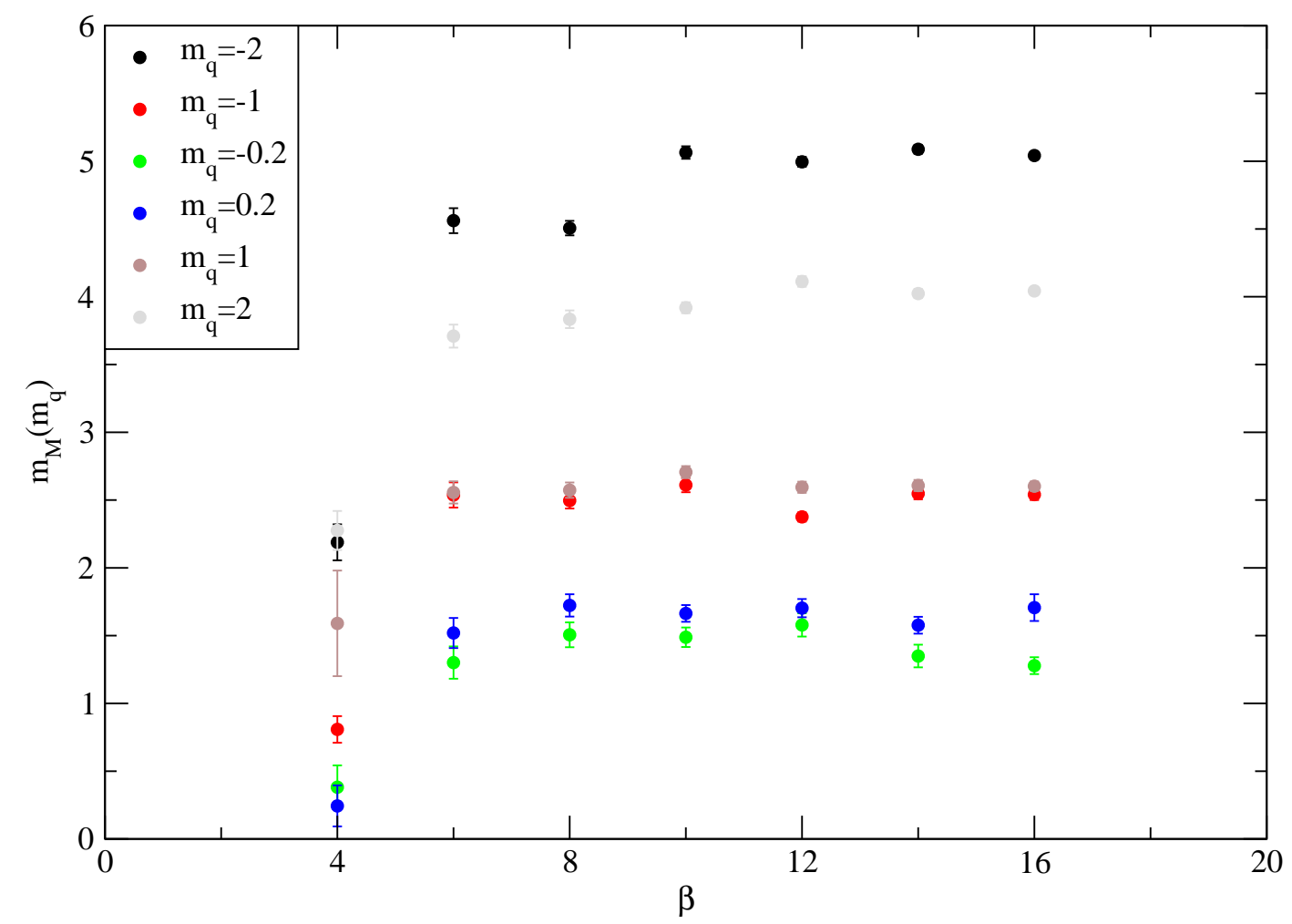

Figure 6.2: A plot of $m_{M}\left(m_{q}\right)$ as a function of $\beta$ at $L=6$ and $b=0.25$ at six different sample values of $m_{q}$. 


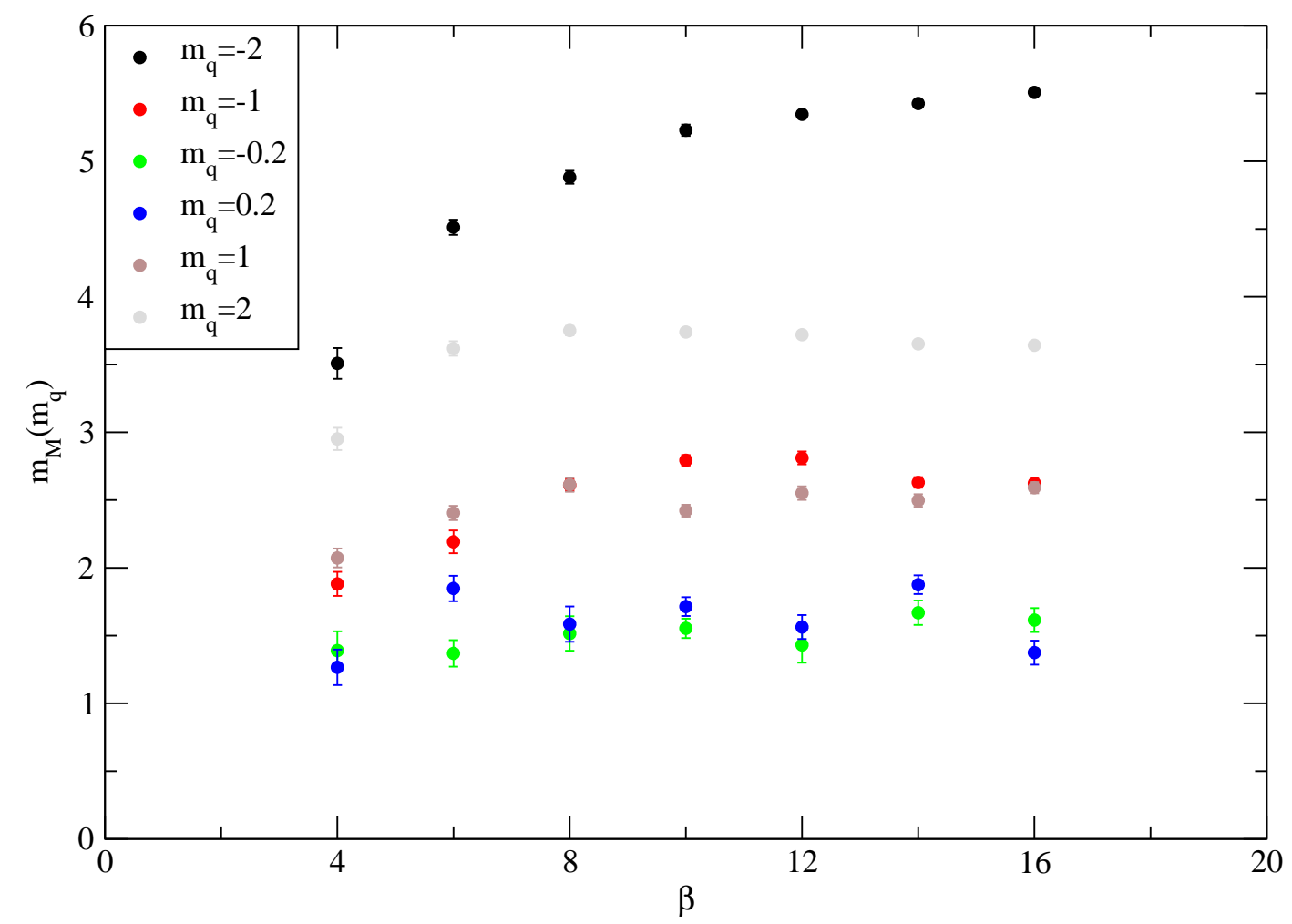

Figure 6.3: A plot of $m_{M}\left(m_{q}\right)$ as a function of $\beta$ at $L=4$ and $b=0.5$ at six different sample values of $m_{q}$. 


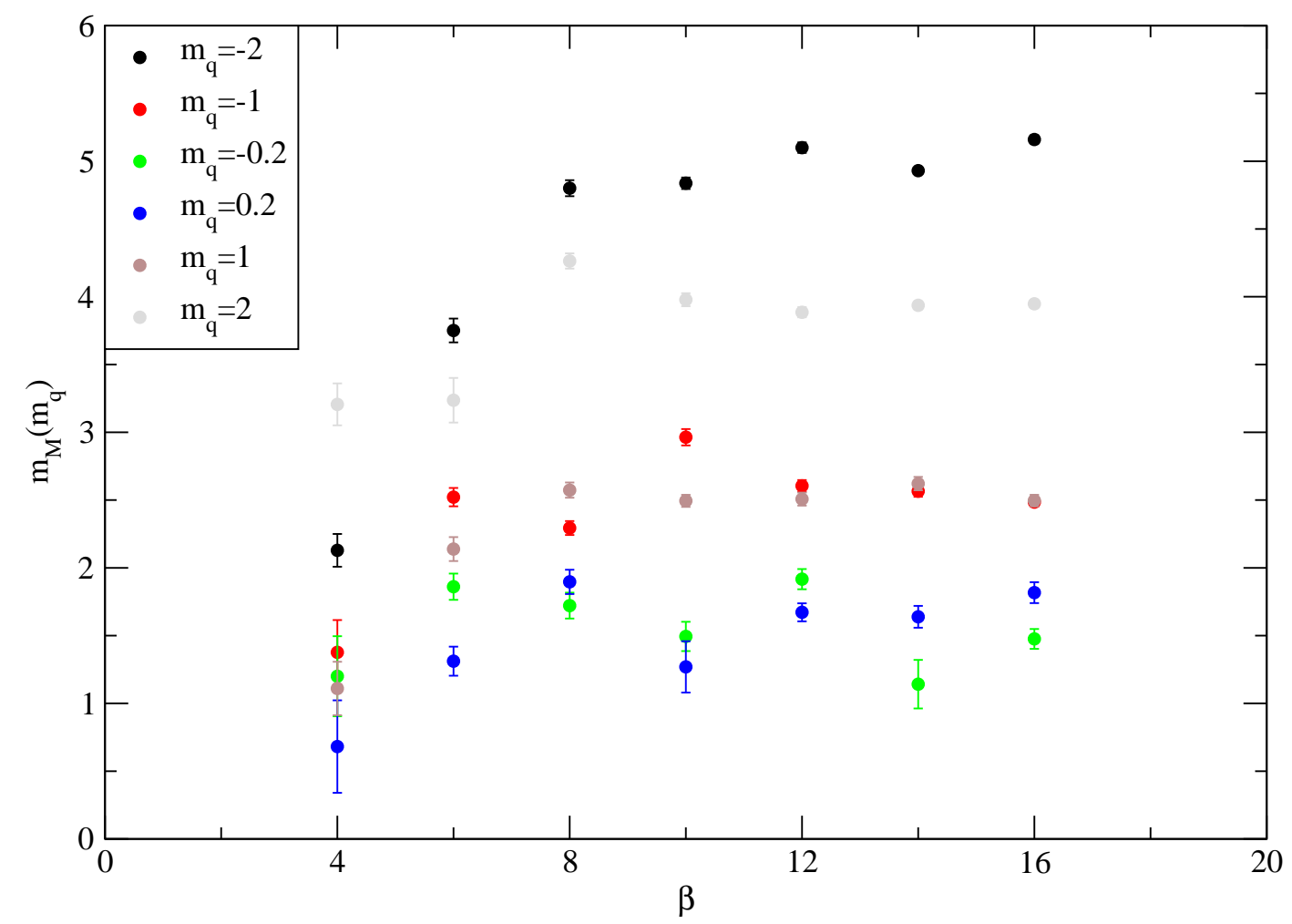

Figure 6.4: A plot of $m_{M}\left(m_{q}\right)$ as a function of $\beta$ at $L=6$ and $b=0.5$ at six different sample values of $m_{q}$. 


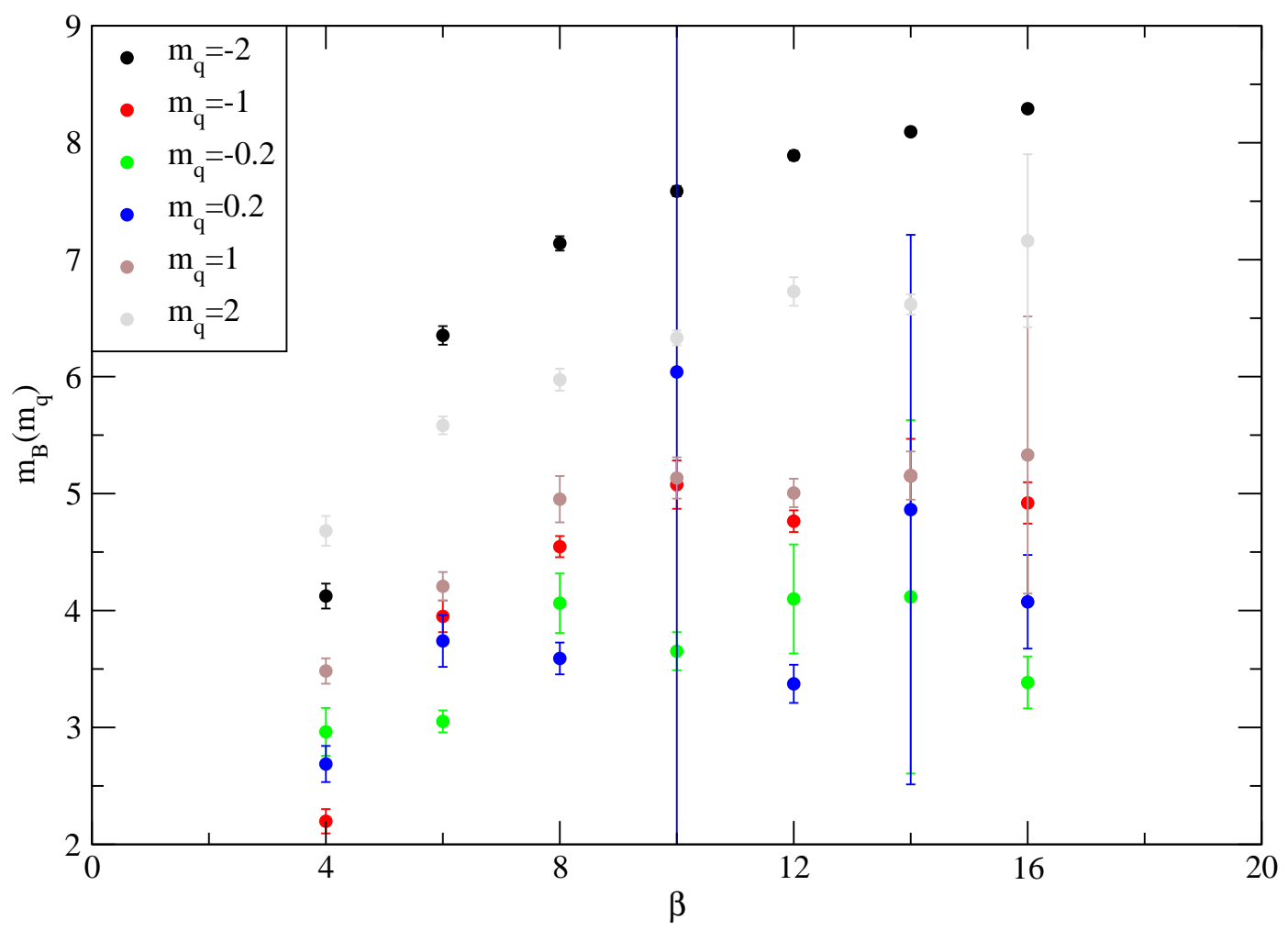

Figure 6.5: A plot of $m_{B}\left(m_{q}\right)$ as a function of $\beta$ at $L=4$ and $b=0.25$ at six different sample values of $m_{q}$. 


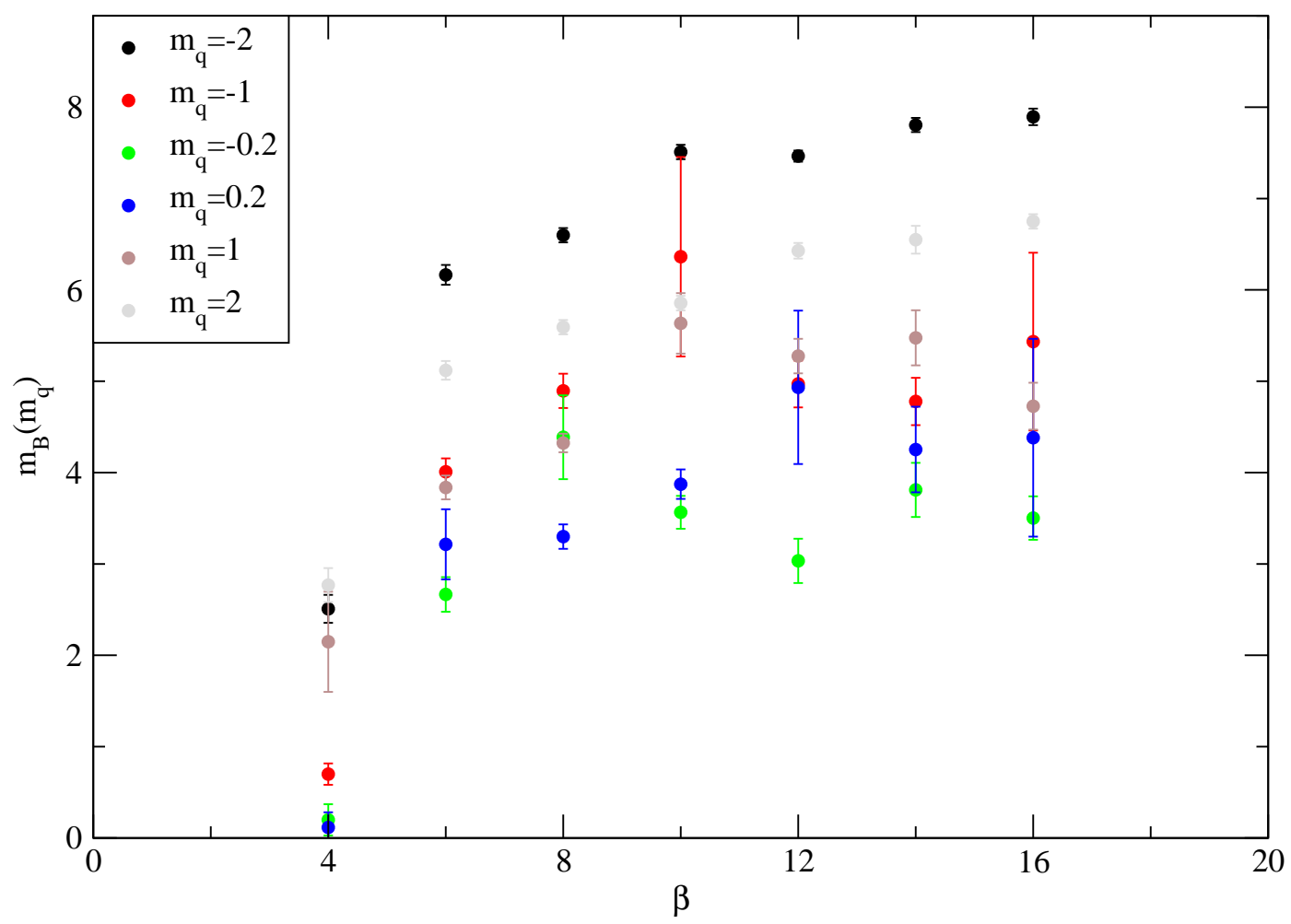

Figure 6.6: A plot of $m_{B}\left(m_{q}\right)$ as a function of $\beta$ at $L=6$ and $b=0.25$ at six different sample values of $m_{q}$. 


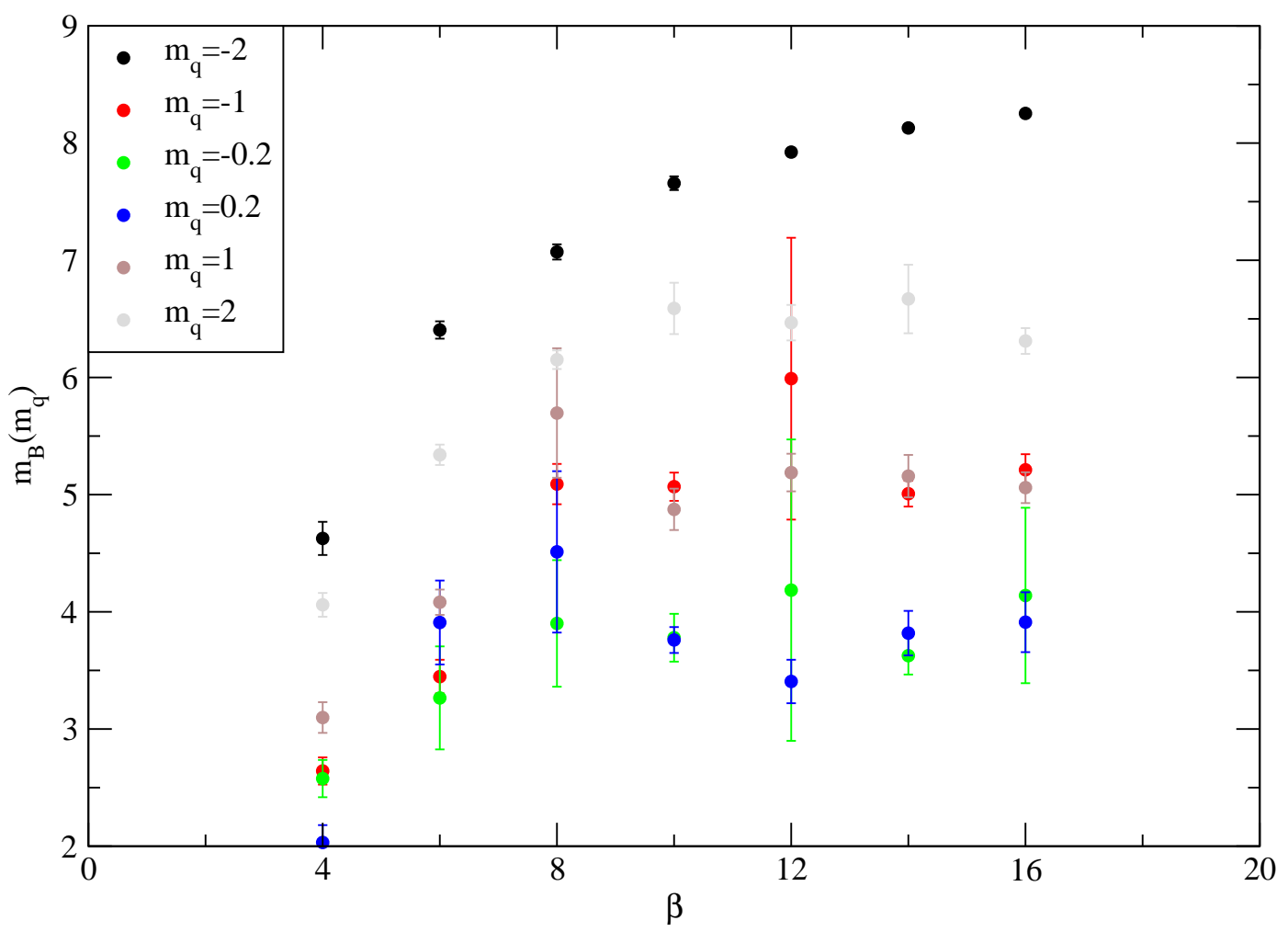

Figure 6.7: A plot of $m_{B}\left(m_{q}\right)$ as a function of $\beta$ at $L=4$ and $b=0.5$ at six different sample values of $m_{q}$. 


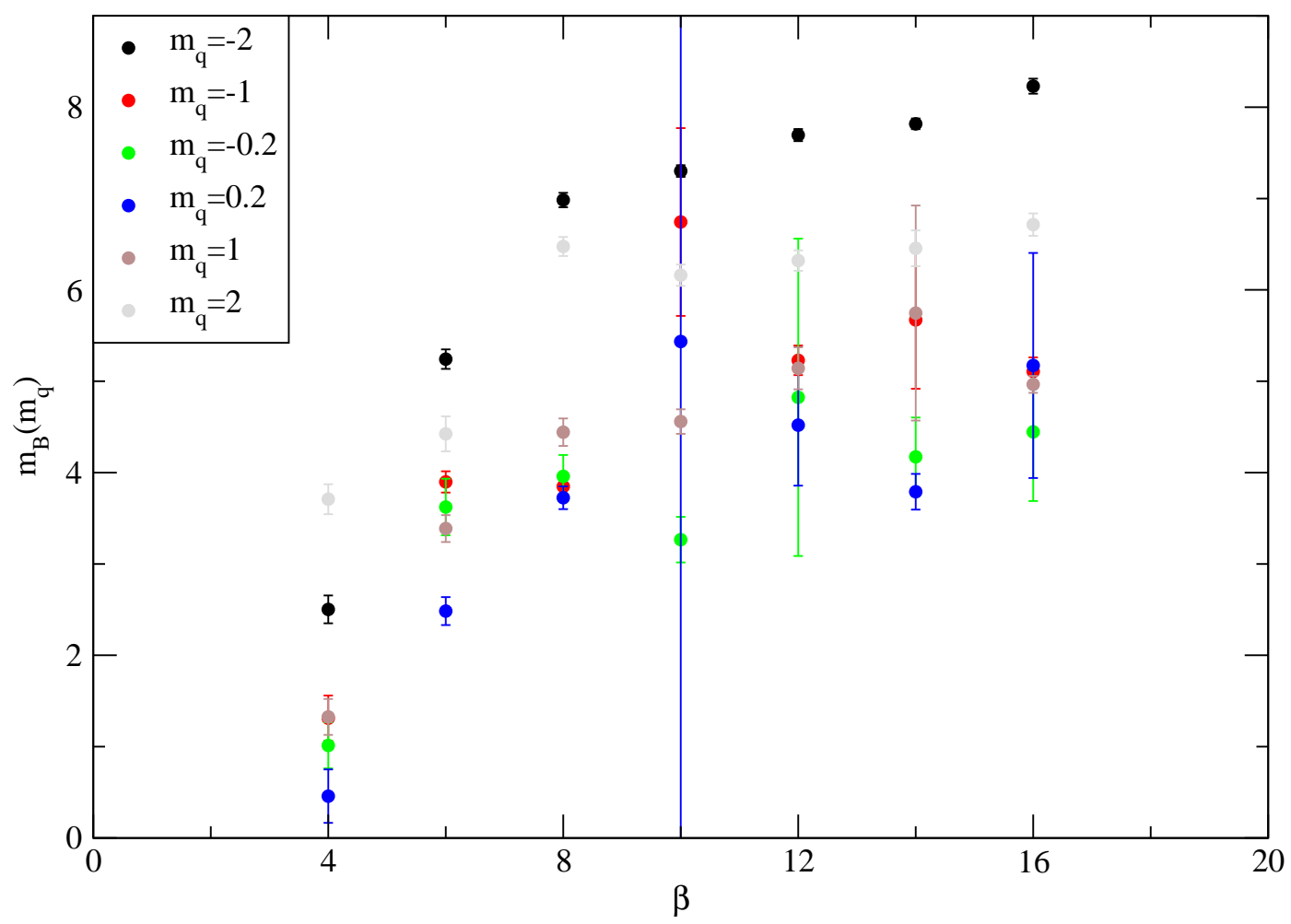

Figure 6.8: A plot of $m_{B}\left(m_{q}\right)$ as a function of $\beta$ at $L=6$ and $b=0.5$ at six different sample values of $m_{q}$. 


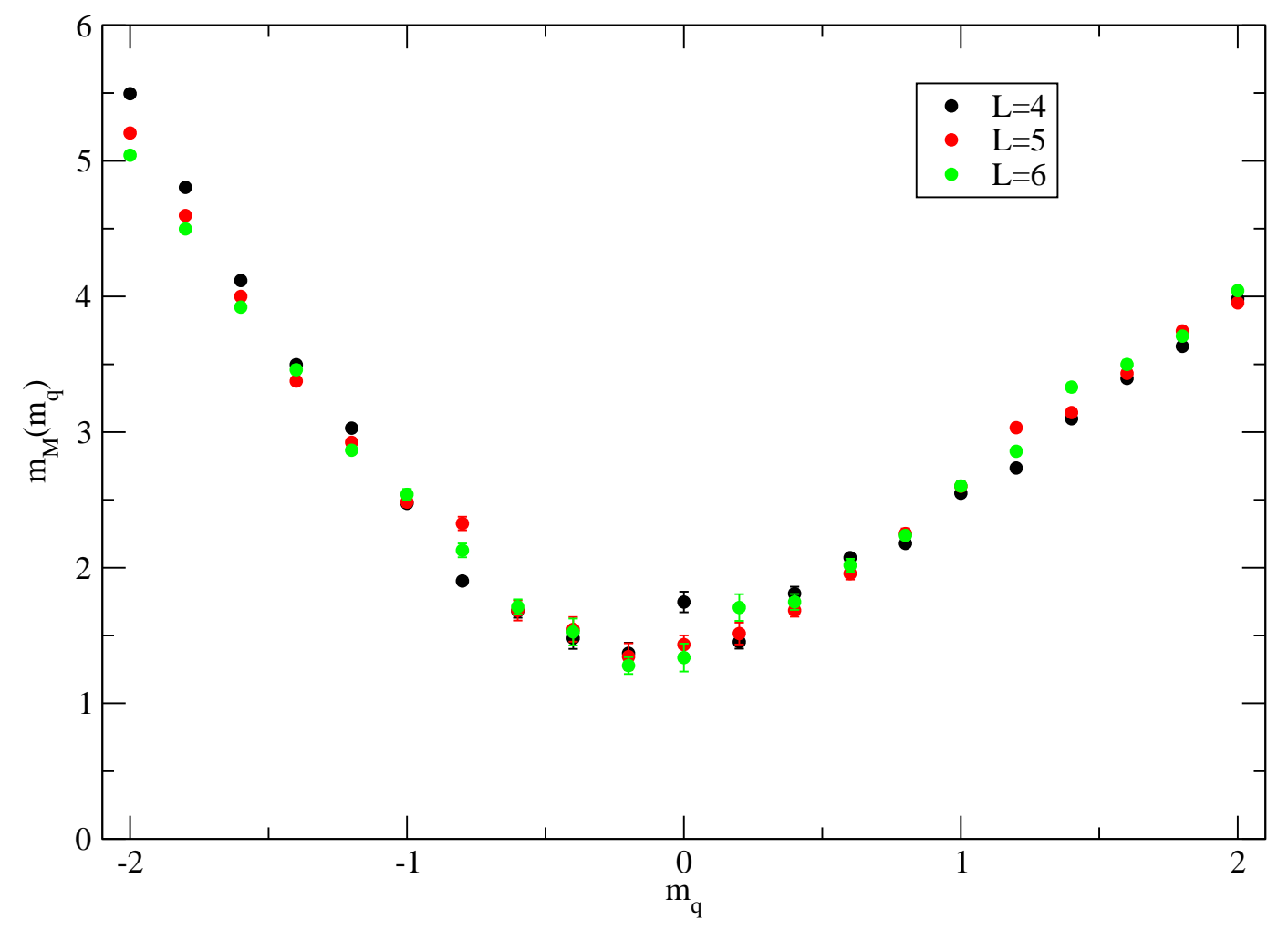

Figure 6.9: A plot of $m_{M}\left(m_{q}\right)$ as a function of $m_{q}$ at $b=0.25$ for $L=4,5,6$. 


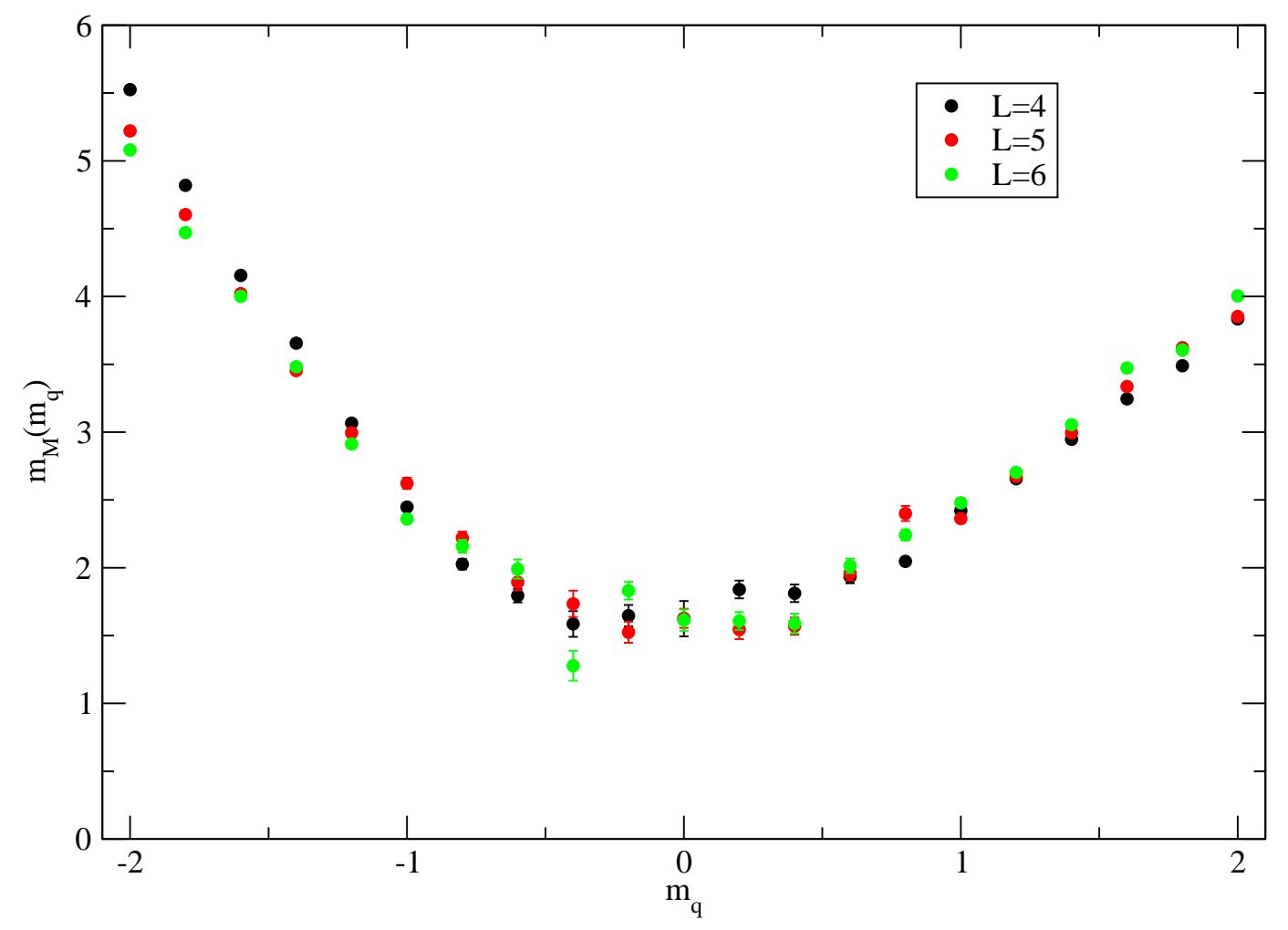

Figure 6.10: A plot of $m_{M}\left(m_{q}\right)$ as a function of $m_{q}$ at $b=0.375$ for $L=4,5,6$. 


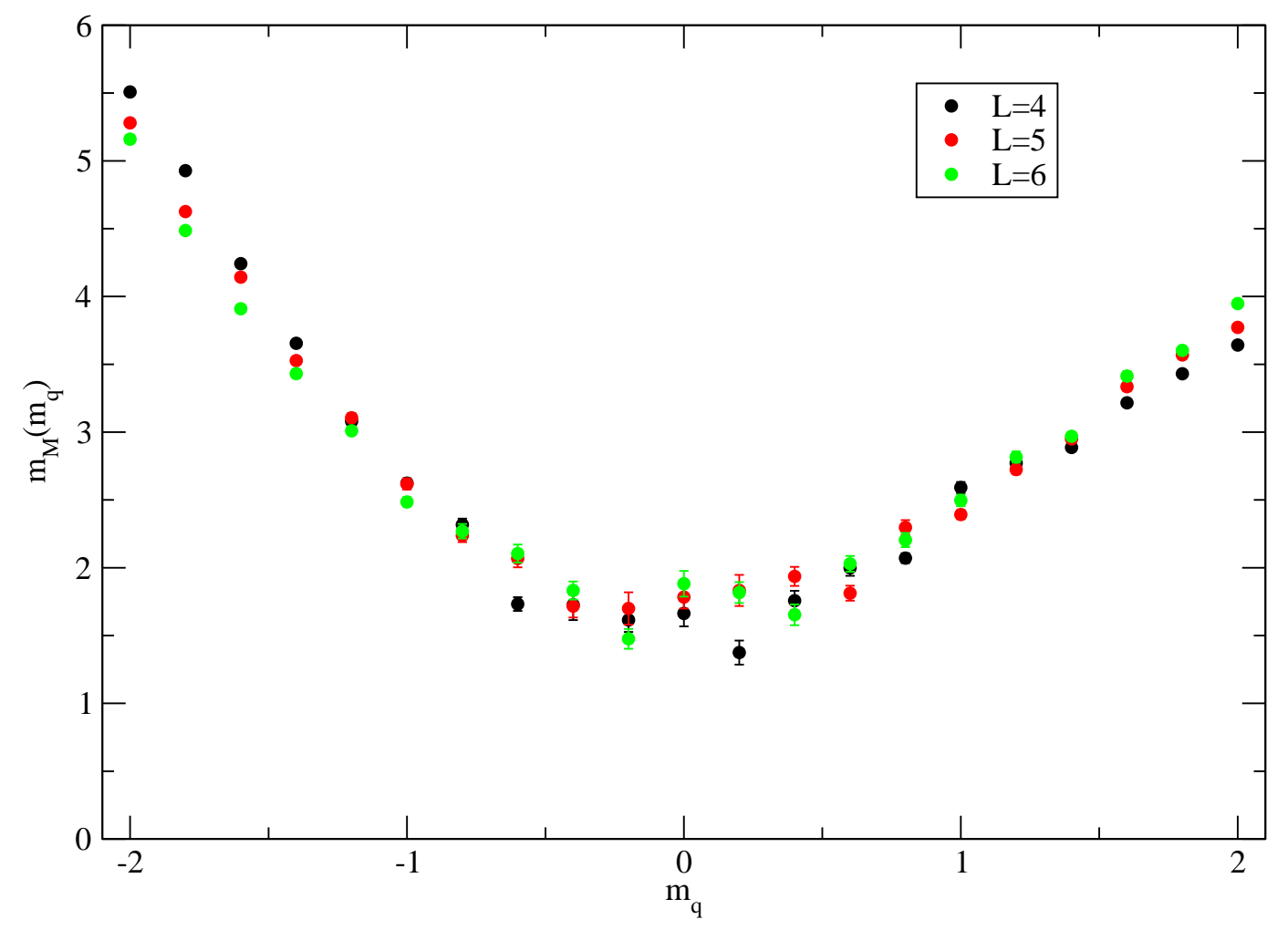

Figure 6.11: A plot of $m_{M}\left(m_{q}\right)$ as a function of $m_{q}$ at $b=0.5$ for $L=4,5,6$. 


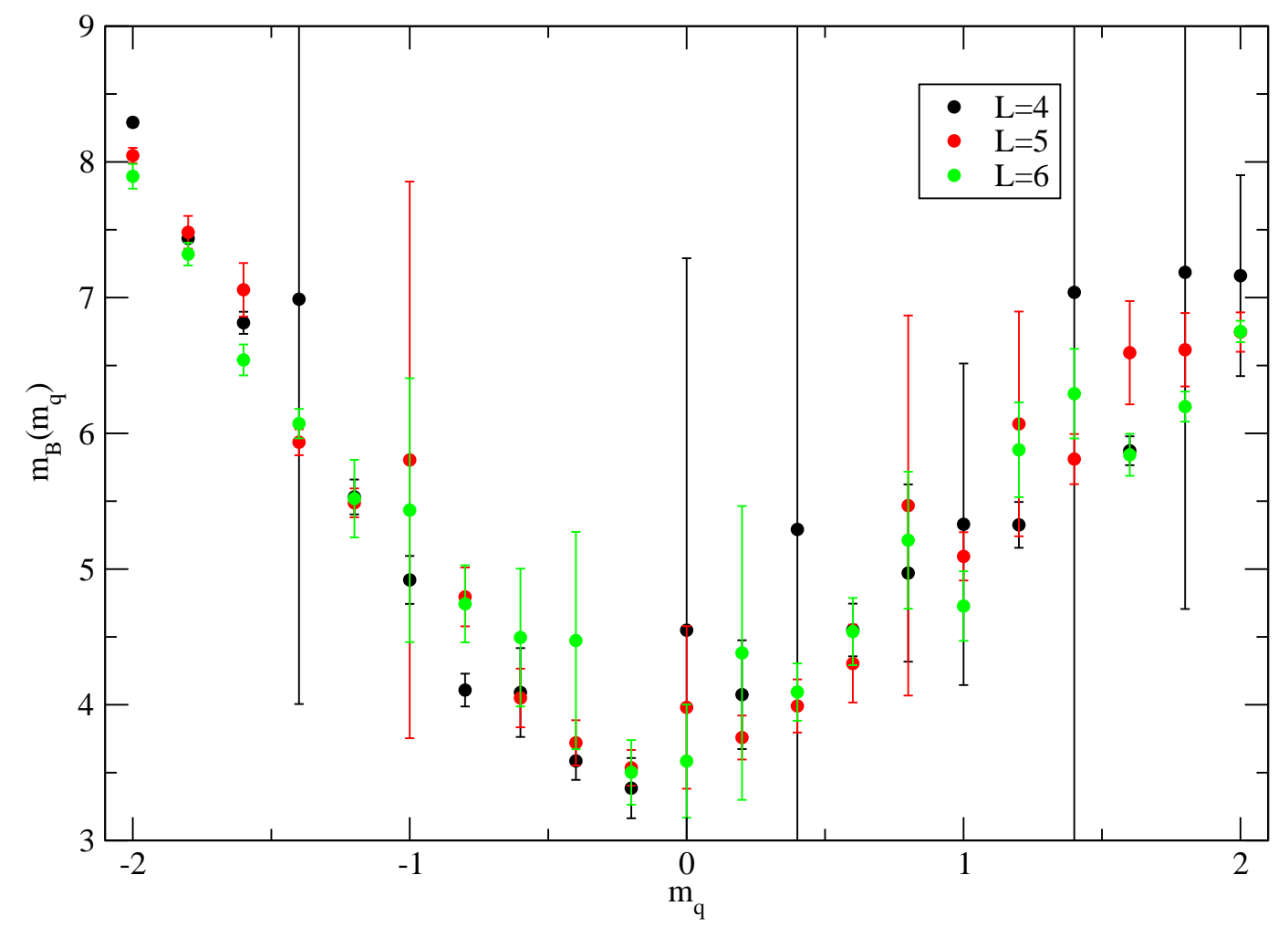

Figure 6.12: A plot of $m_{B}\left(m_{q}\right)$ as a function of $m_{q}$ at $b=0.25$ for $L=4,5,6$. 


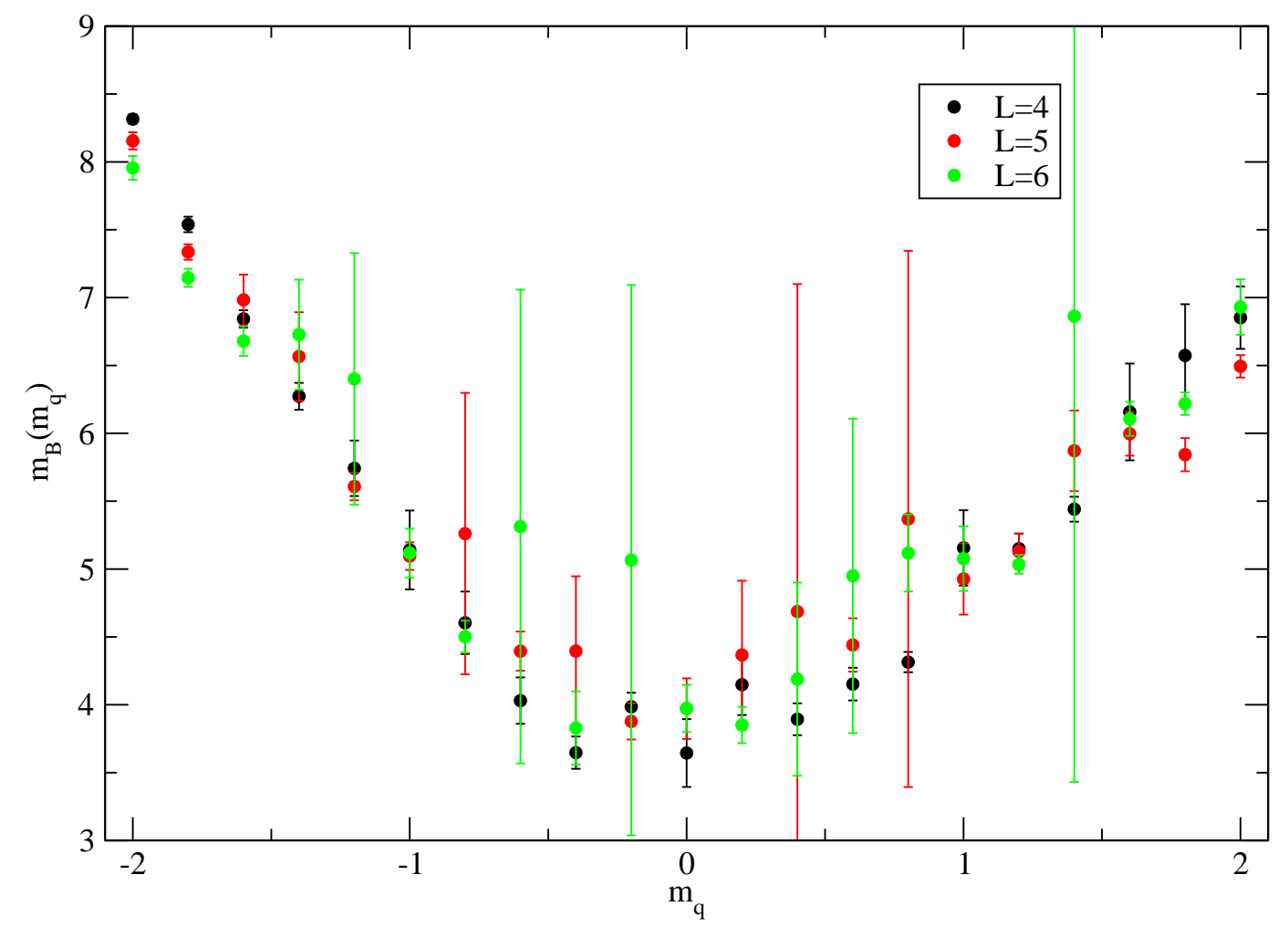

Figure 6.13: A plot of $m_{B}\left(m_{q}\right)$ as a function of $m_{q}$ at $b=0.375$ for $L=4,5,6$. 


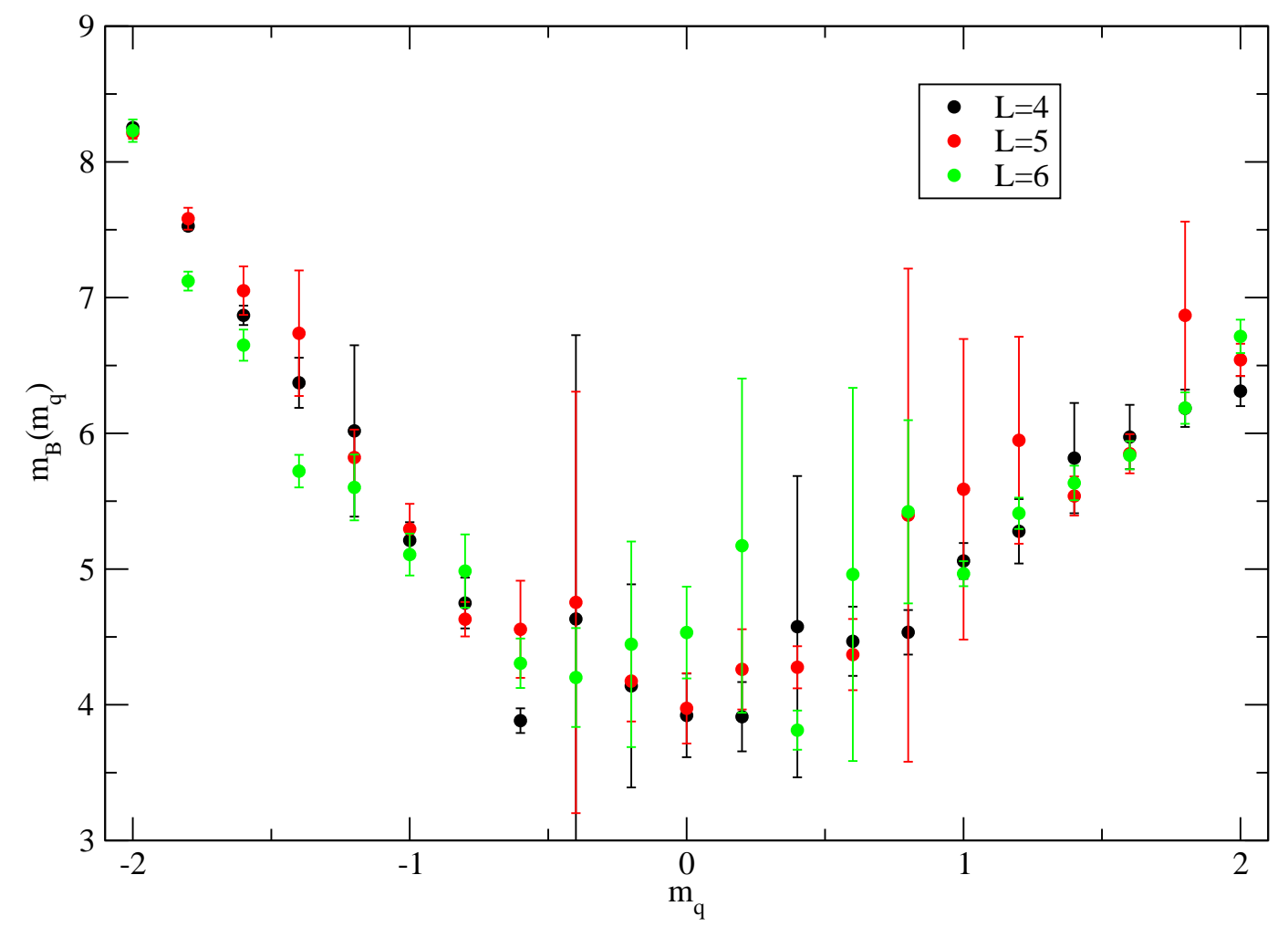

Figure 6.14: A plot of $m_{B}\left(m_{q}\right)$ as a function of $m_{q}$ at $b=0.5$ for $L=4,5,6$. 


\subsection{Extraction of the continuum limit}

The data shown in figure 6.15 for the pion mass as a function of the quark mass are the same as the ones in figures $6.9,6.10$ and 6.11 restricted to $L=6$. The data shown in figure 6.16 for the baryon mass as a function of the quark mass are the same as the ones in figures $6.12,6.13$ and 6.14 restricted to $L=6$.

In order to extract the continuum limit, we consider the following ansatz:

$$
\begin{aligned}
& m_{M}\left(m_{q}\right)=m_{M}(0)+\alpha_{M} m_{q}+\beta_{M} m_{q}^{2}+\gamma_{M} m_{q}^{3} \\
& m_{B}\left(m_{q}\right)=m_{B}(0)+\alpha_{B} m_{q}+\beta_{B} m_{q}^{2}+\gamma_{B} m_{q}^{3} .
\end{aligned}
$$

A standard least square fit of the data was used to extract the coefficients listed in Table 6.1 and 6.2 for pion and baryon respectively. In addition, plots using the mean values of the coefficients are shown in figures 6.15 and 6.16 with continuous lines and the colors of the lines match the data.

The three values of $m_{M}(0)$ shown in Table 6.1 for the three different values of $b$ are consistent with each other within one standard deviation indicating that $m_{M}(0)=1.75(7)$ is a reliable value in the continuum. The three values of $\beta_{M}$ shown in Table 6.1 for the three different values of $b$ show a small downward trend as $b$ is increased but the value in the continuum is expected to be different from zero. Since we have data only at three different values of $b$ we do not perform a fit as a function of $b$ to extract the continuum limit. There is reasonably good evidence that $\alpha_{M}$ is consistent with zero in the continuum limit since the value shows a large drop in the region of $b$ studied here. The same cannot be said of $\gamma_{M}$ with such confidence but it is also probably zero in the continuum limit. These plots would suggest a dependence of $m_{M}\left(m_{q}\right)$ that is explicitly an even function with a non-zero $m_{M}(0)$ and the presence of a quadratic term.

In spite of the large error bars in the baryon data which results in large error bars on the coefficients, the three values of $m_{B}(0)$ shown in 6.2 for the three different values of $b$ are consistent with each other within one standard deviation indicating that $m_{B}(0)=4.4(5)$ 
is a reliable value in the continuum. The three values of $\beta_{B}$ shown in Table 6.2 for the three different values of $b$ show a small downward trend as $b$ is increased but the value in the continuum is expected to be different from zero. Since we have data only at three different values of $b$ we do not perform a fit as a function of $b$ to extract the continuum limit. Unlike the behavior of the meson mass, we cannot say with any confidence if $\alpha_{B}$ and $\gamma_{B}$ are different from zero in the continuum. The coefficients themselves are small but we cannot conclude that the baryon mass is an even function of the quark mass.

Table 6.1: The values of the coefficients obtained from the fit of the pion mass data to the ansatz in equation (6.2)

\begin{tabular}{lllll}
\hline \hline$b$ & $m_{M}(0)$ & $\alpha_{M}$ & $\beta_{M}$ & $\gamma_{M}$ \\
\hline 0.25 & $1.61(7)$ & $0.178(5)$ & $0.787(14)$ & $-0.1141(15)$ \\
0.375 & $1.65(6)$ & $0.077(6)$ & $0.759(13)$ & $-0.0924(19)$ \\
0.5 & $1.75(7)$ & $0.032(3)$ & $0.719(13)$ & $-0.084(8)$ \\
\hline
\end{tabular}

Table 6.2: The values of the coefficients obtained from the fit of the baryon mass data to the ansatz in equation (6.2)

\begin{tabular}{lllll}
\hline \hline$b$ & $m_{B}(0)$ & $\alpha_{B}$ & $\beta_{B}$ & $\gamma_{B}$ \\
\hline 0.25 & $4.2(5)$ & $0.15(8)$ & $0.83(13)$ & $-0.119(25)$ \\
0.375 & $4.4(8)$ & $-0.06(12)$ & $0.80(15)$ & $-0.052(7)$ \\
0.5 & $4.4(5)$ & $0.21(15)$ & $0.72(15)$ & $-0.15(5)$ \\
\hline
\end{tabular}




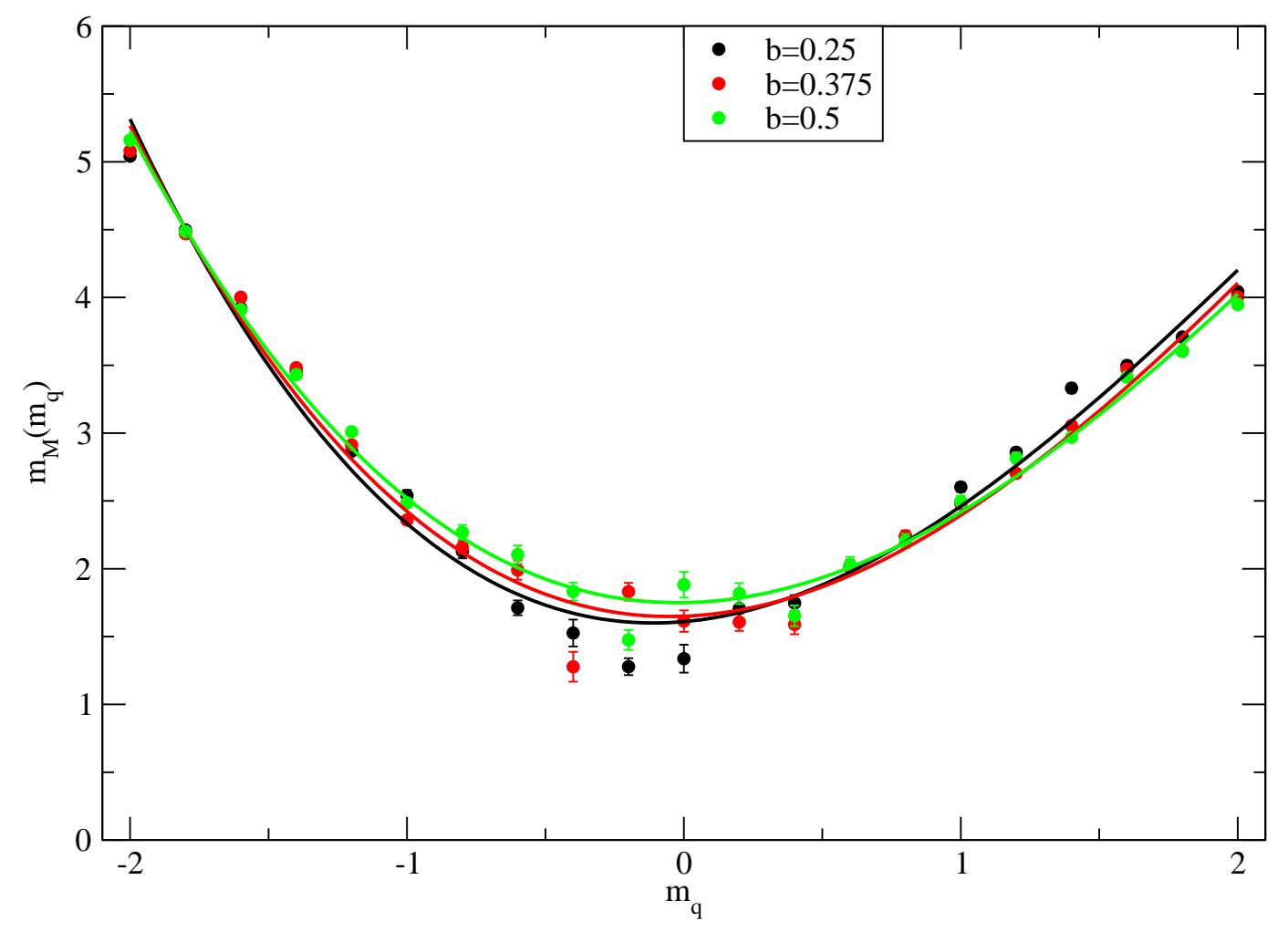

Figure 6.15: A plot of $m_{M}\left(m_{q}\right)$ as a function of $m_{q}$ at $L=6$ for $b=0.25,0.375,0.5$. 


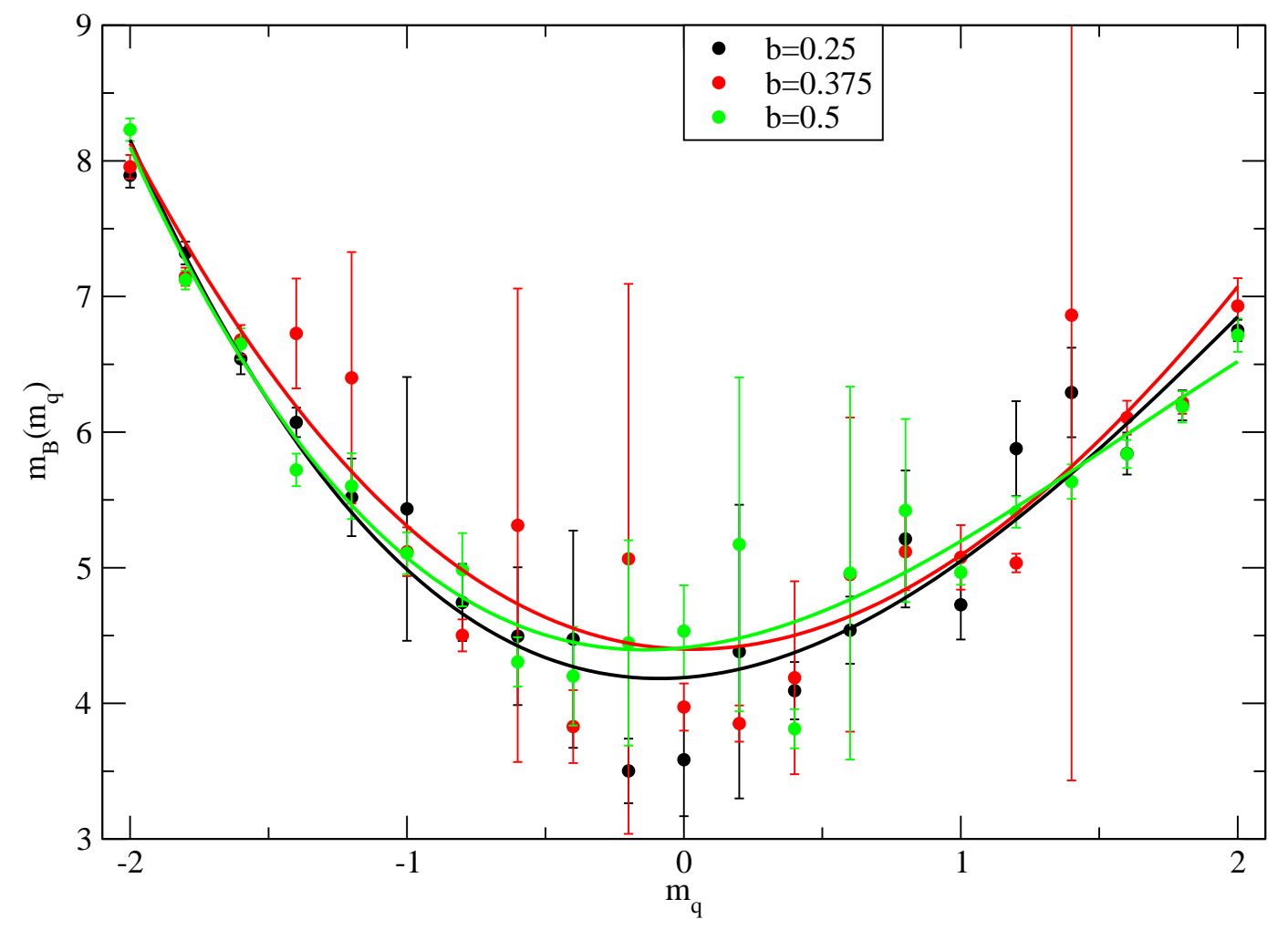

Figure 6.16: A plot of $m_{B}\left(m_{q}\right)$ as a function of $m_{q}$ at $L=6$ for $b=0.25,0.375,0.5$. 


\section{CHAPTER 7}

\section{Conclusion}

Two dimensional QCD with and without fermions has proved to be a useful model to understand the physically relevant four dimensional QCD with fermions. Analytical techniques were used to extensively study observables in two dimensional Yang-Mills theory. Numerical techniques using the lattice formalism were used to understand the physical spectrum of two dimensional QCD with fermions.

The partition function of two dimensional Yang-Mills has a simple expression as an infinite sum over different irreducible representation of the gauge group. Each term in the sum has an associated free energy which is proportional to the area of the two dimensional torus and the proportionality constant depends on the group theory details of the representation. Expectation value of Wilson loops show confinement since the free energy associated with the insertion of a Wilson loop is proportional to the area of the Wilson loop which is assumed to be small compared to the area of the torus. A new result in this thesis is the expectation value of the correlation of two Polyakov operators in two different directions. Due to translational invariance of the theory, this observable only depends on the group representation of the two Polyakov loops. The analytical results obtained for a general gauge group were numerically analyzed for the special case of $\mathrm{SU}(2)$ in order to obtain the energy of the lowest state associated with the insertion of two Polyakov loops in two different directions. As expected, the trivial vacuum has the lowest energy in the limit of infinite area of the torus. Such trivial vacuum with lowest energy is not the case

when the area of the torus is finite. A specific Polykov loop operator obtained by choosing a certain linear combination of all representations has the lowest energy and this represents the true vacuum of two dimensional Yang-Mills theory. We obtained the numerical value for the vacuum energy as a function of the area of the two dimensional torus.

In order to study two dimensional QCD with fermion content, a certain form of the Hamiltonian formalism of lattice gauge theory was devloped using the "axial" gauge on the torus. The partition function is ontained as an infinite sum where each term in the 
sum has an associated quark number and baryon number. An expression for the meson and baryons masses were obtained in terms of the quark excitations out of the vacuum. The Hybrid Monte Carlo technique was used to generate important gauge field configurations and all quark excitations were computed in each gauge field background. Using this information, meson and baryon masses were computed on the lattice at a finite spatial extent. Using results at several different lattice spacings, meson and baryons masses in the continuum limit were extracted at a finite spatial extent. These were used to extract the corresponding masses in infinite spatial extent. Both meson masses and baryon masses increased with increasing quark mass. Both the lowest meson mass and the lowest baryon mass remained non-zero for zero quark mass clearly establishing the presence of a mass gap in two dimensional QCD.

In the chapter 5, two dimensional QCD with fermion extent was studied with the aim of extracting meson and baryon masses with $\mathrm{SU}(3)$ gauge fields and two degenerate flavors of quarks. For this purpose, it was convenient to use the canonical formalism. After providing the details of the canonical formalism, a numerical algorithm for the computation of the meson and baryon masses was presented. The numerical data so obtained was used to extract the behavior of the meson and baryon masses as a function of quark masses which was presented systematically in chapter 6 . The dimensionless lowest meson mass at zero quark mass was found to be 1.75(7) and the dimensionless lowest baryon mass at zero quark mass was found to be 4.4(5). Since a meson is made up of two quarks, the effective mass of a single quark in a meson with massless quarks is $0.87(4)$ and the effective mass of a single quark in a baryon with massless quarks in 1.5(3). In other words the effective quarks appear quite a bit heavier inside a baryon compared to a meson like in the physically relevant four dimensional QCD. The investigation of meson and baryon masses for negative quark masses showed that the sign of the quark mass is not physically relevant. Due to numerical uncertainities, this result was more convicing for mesons then that for baryons.

In conclusion, two dimensional QCD was used to successfully study the vacuum structure and the physical spectrum which shed some insight into the physically relevant four dimensional QCD. 
Bibliography

[1] J. Beringer et al. Review of Particle Physics (RPP). Phys. Rev., D86:010001, 2012.

[2] S. Weinberg. The Quantum Theory of Fields. Number v. 1 in The Quantum Theory of Fields 3 Volume Hardback Set. Cambridge University Press, 1995.

[3] S. Weinberg. The Quantum Theory of Fields: Volume 2, Modern Applications. Cambridge University Press, 1996.

[4] M.E. Peskin and D.V. Schroeder. An Introduction to Quantum Field Theory. Advanced book program. Levant Books, 2005.

[5] David J. Gross, Robert D. Pisarski, and Laurence G. Yaffe. QCD and Instantons at Finite Temperature. Rev. Mod. Phys., 53:43, 1981.

[6] Maria Paola Lombardo. QCD at finite temperature and density on the lattice. EPJ Web Conf., 7:01004, 2010.

[7] Kenneth G. Wilson. Confinement of Quarks. Phys. Rev., D10:2445-2459, 1974. $[, 45(1974)]$.

[8] I. Montvay and G. Münster. Quantum Fields on a Lattice. Cambridge Monographs on Mathematical Physics. Cambridge University Press, 1997.

[9] T. DeGrand and C. DeTar. Lattice Methods for Quantum Chromodynamics. World Scientific, 2006.

[10] Gerard 't Hooft. A Two-Dimensional Model for Mesons. Nucl. Phys., B75:461-470, 1974.

[11] Curtis G. Callan, Jr., Nigel Coote, and David J. Gross. Two-Dimensional Yang-Mills Theory: A Model of Quark Confinement. Phys. Rev., D13:1649, 1976.

[12] G. Parisi. Statistical Field Theory. Advanced book classics. Perseus Books, 1998.

[13] J. Zinn-Justin. Quantum Field Theory and Critical Phenomena. International series of monographs on physics. Clarendon Press, 2002.

[14] C. Itzykson and J.M. Drouffe. Statistical Field Theory: Volume 1, From Brownian Motion to Renormalization and Lattice Gauge Theory. Cambridge Monographs on Mathematical Physics. Cambridge University Press, 1991.

[15] C. Itzykson and J.M. Drouffe. Statistical Field Theory: Volume 2, Strong Coupling, Monte Carlo Methods, Conformal Field Theory and Random Systems. Cambridge Monographs on Mathematical Physics. Cambridge University Press, 1991.

[16] Alexander A. Migdal. Recursion Equations in Gauge Theories. Sov. Phys. JETP, 42:413, 1975. [Zh. Eksp. Teor. Fiz.69,810(1975)].

[17] David J. Gross and Washington Taylor. Two-dimensional QCD is a string theory. Nucl. Phys., B400:181-208, 1993. 
[18] R.P. Feynman, A.R. Hibbs, and D.F. Styer. Quantum Mechanics and Path Integrals. Dover Books on Physics. Dover Publications, 2010.

[19] Curtis G. Callan, Jr. Broken scale invariance in scalar field theory. Phys. Rev., D2:15411547, 1970.

[20] K. Symanzik. Small distance behavior in field theory and power counting. Commun. Math. Phys., 18:227-246, 1970.

[21] K. Symanzik. Small distance behavior analysis and Wilson expansion. Commun. Math. Phys., 23:49-86, 1971.

[22] Andreas Petermann. La normalisation des constantes dans la thorie des quantaNormalization of constants in the quanta theory. Helv. Phys. Acta, 26:499-520, 1953.

[23] Murray Gell-Mann and F. E. Low. Quantum electrodynamics at small distances. Phys. Rev., 95:1300-1312, 1954.

[24] D. J. Gross and Edward Witten. Possible Third Order Phase Transition in the Large N Lattice Gauge Theory. Phys. Rev., D21:446-453, 1980.

[25] Tohru Eguchi and Hikaru Kawai. Reduction of Dynamical Degrees of Freedom in the Large N Gauge Theory. Phys. Rev. Lett., 48:1063, 1982.

[26] M. Creutz. Quarks, Gluons and Lattices. Cambridge Monographs on Mathematical Physics. Cambridge University Press, 1983.

[27] Joe Kiskis, Rajamani Narayanan, and Dibakar Sigdel. Correlation between Polyakov loops oriented in two different directions in $\mathrm{SU}(\mathrm{N})$ gauge theory on a two dimensional torus. Phys. Rev., D89(8):085031, 2014.

[28] M. Hamermesh. Group Theory and Its Application to Physical Problems. Addison Wesley Series in Physics. Dover Publications, 1962.

[29] Jean-Michel Drouffe and Jean-Bernard Zuber. Strong Coupling and Mean Field Methods in Lattice Gauge Theories. Phys. Rept., 102:1, 1983.

[30] C. Gattringer and C. Lang. Quantum Chromodynamics on the Lattice: An Introductory Presentation. Lecture Notes in Physics. Springer Berlin Heidelberg, 2009.

[31] Herbert Neuberger. Vector - like gauge theories with almost massless fermions on the lattice. Phys. Rev., D57:5417-5433, 1998.

[32] S. Duane, A. D. Kennedy, B. J. Pendleton, and D. Roweth. Hybrid Monte Carlo. Phys. Lett., B195:216-222, 1987. 
VITA

DIBAKAR SIGDEL

$2003-2006$

$2006-2008$

$2011-2017$
B.Sc., Physics

Tribhuvan University

Kathmandu, Nepal

M.Sc., Physics

Tribhuvan University

Kathmandu, Nepal

Ph.D., Physics

Florida International University

Miami, Florida

\section{SELECTED PUBLICATIONS}

1. Joe Kiskis, Rajamani Narayanan, and Dibakar Sigdel, Correlation between Polyakov loops oriented in two different directions in $S U(N)$ gauge theory on a two-dimensional torus, Phys. Rev. D 89, 08503116 April 2014 